\title{
$d$-wave correlated critical Bose liquids in two dimensions
}

\author{
Olexei I. Motrunich \\ Department of Physics, California Institute of Technology, Pasadena, California 91125, USA \\ Matthew P. A. Fisher \\ Kavli Institute for Theoretical Physics, University of California, Santa Barbara, California 93106, USA
}

(Received 21 March 2007; published 21 June 2007)

\begin{abstract}
We develop a description of a quantum liquid phase of interacting bosons confined in two dimensions that possesses relative $d$-wave two-body correlations. We refer to this stable quantum phase as a $d$-wave Bose liquid (DBL). The DBL has no broken symmetries, supports gapless boson excitations that reside on "Bose surfaces" in momentum space, and exhibits power-law correlation functions characterized by a manifold of continuously variable exponents. While the DBL can be constructed for bosons moving in the two-dimensional continuum, the state only respects the point group symmetries of the square lattice. On the square lattice, the DBL respects all symmetries and does not require a particular lattice filling. However, lattice effects do allow for the possibility of a second distinct phase, a quasilocal variant we refer to as a $d$-wave local Bose liquid (DLBL). Remarkably, the DLBL has short-range boson correlations and hence no Bose surfaces, despite sharing gapless excitations and other critical signatures with the DBL. Moreover, both phases are metals with a resistance that vanishes as a power of the temperature. We establish these results by constructing a class of many-particle wave functions for the DBL, which are time reversal invariant analogs of Laughlin's quantum Hall wave function for bosons in a half-filled Landau level. A gauge theory formulation leads to a simple mean field theory, and a suitable $N$-flavor generalization enables incorporation of gauge field fluctuations to deduce the properties of the DBL/DLBL in a controlled and systematic fashion. Various equal-time correlation functions thereby obtained are in qualitative accord with the properties inferred from the variational wave functions. We also identify a promising microscopic Hamiltonian that might manifest the DBL or DLBL, and perform a variational energetics study comparing other competing phases, including the superfluid. We suggest how the $d$-wave Bose liquid wave function can be suitably generalized to describe an itinerant non-Fermi-liquid phase of electrons on the square lattice with a no-double-occupancy constraint, a $d$-wave metal phase.
\end{abstract}

DOI: 10.1103/PhysRevB.75.235116

PACS number(s): 71.10.Hf, 71.10.Pm, 74.20.Mn, 75.10.Jm

\section{INTRODUCTION}

The principal roadblock impeding progress in disentangling the physics of the cuprate superconductors is arguably our inability to access quantum ground states of twodimensional itinerant electrons which are qualitatively distinct from a Landau Fermi liquid. Overcoming this obstruction is of paramount importance, indispensable in explaining the strange metal behavior observed near optimal doping and a likely requisite to account for the emergent pseudogap at lower energies. A putative underlying paramagnetic Mott insulator provides the scaffolding for a popular class of theories, which view the pseudogap as a lightly doped spin liquid. ${ }^{1-5}$ Significant progress has been made in developing the groundwork and there now exists a well established theoretical framework to describe a myriad of distinct spin liquids. For the cuprates, the most promising spin liquids are described in terms of fermionic spinons minimally coupled to a compact U(1) gauge field and moving in various background fluxes. Upon doping, formidable challenges arise. Bosonic holons carrying the electron charge become mobile carriers and lead to electrical conduction. However, at low temperatures, Bose condensation appears inevitable and this leads to Fermi-liquid behavior, either a metal with conventional Landau quasiparticles or a BCS superconductor if the spinons are paired. Accessing a pseudogap or a strange metal which conduct electricity despite the absence of long-lived
Landau quasiparticles requires doped holons that form an uncondensed quantum Bose fluid rather than a condensed superfluid. However, is this possible, even in principle? If possible, what properties would such a putative "Bose metal" exhibit? ${ }^{6-9}$ What theoretical framework is appropriate? The slave-particle gauge theory approach has been stymied by this stumbling block for over 15 years. In this paper, we provide (some) answers to these questions by constructing explicit examples of such unusual phases of bosons that may offer some routes out of the conundrum.

Our goal, then, is to access and explore uncondensed quantum phases of two-dimensional (2D) bosons which are conducting fluids but not superfluids. Specifically, we have in mind hard-core bosons moving on a 2D square lattice, but seek to construct states which do not require particular commensurate densities. While our construction can be implemented for bosons moving in the 2D continuum Euclidean plane, the states will only possess the reduced point group symmetry of the square lattice. Despite our interest in time reversal invariant quantum ground states, our technical approach will be strongly informed by theories of the fractional quantum Hall effect (FQHE). In some regards, the quantum phases that we construct are time reversal invariant analogs of the Laughlin state for bosons in a half-filled Landau level. However, the physical properties of the phases will be dramatically different from the incompressible FQHE states, and will have gapless excitations and metallic transport, for example. 
To motivate and illustrate our approach, it will be helpful to briefly revisit the bosonic FQHE. Consider the Laughlin wave function for bosons in a half-filled Landau level,

$$
\Psi_{\nu=1 / 2}\left(z_{1}, z_{2}, \ldots, z_{N}\right)=\prod_{i<j}\left(z_{i}-z_{j}\right)^{2} .
$$

Much of the physics of the Laughlin state has its origin in the structure of zeroes, which reveals that any two particles upon close approach in real space are in a relative two-body $d$ $+i d$ state $\left[\left(z_{i}-z_{j}\right)^{2}\right]$. Our first objective is to construct a time reversal invariant (real) wave function in which particle pairs are similarly in a relative $d_{x y}$ state. A clue is offered by noting that the Laughlin wave function is the square of a Vandermonde determinant, $\Psi_{\nu=1 / 2}=(\operatorname{det} \mathbf{V})^{2}$. In the Vandermonde determinant, all pairs of particles are in a relative $p$ $+i p$ state $\left[\left(z_{i}-z_{j}\right)\right]$. Upon squaring, the two $p+i p$ states combine to form a single $d+i d$ state, which is essentially just the addition of angular momentum.

This suggests constructing a time reversal invariant boson wave function in zero magnetic field by simply squaring a determinant constructed from momentum states within a Fermi sea,

$$
\Psi\left(\mathbf{r}_{1}, \mathbf{r}_{2}, \ldots, \mathbf{r}_{N}\right)=\left(\operatorname{det} e^{i \mathbf{k}_{i} \cdot \mathbf{r}_{j}}\right)^{2} \quad(S \text { type }) .
$$

Let us examine the nodal structure. The generic behavior of each fermion determinant when any two particles are taken close together is dictated by Fermi statistics and the reality of the wave function, and has the functional form $\left(\mathbf{r}_{i}-\mathbf{r}_{j}\right) \cdot \hat{l}$. The unit vector $\hat{\boldsymbol{l}}$ will depend in a complicated way on the location of all the other particles (see Ref. 10 for a discussion and illustrations of the free fermion nodes). When $\hat{\boldsymbol{l}}=\hat{\mathbf{y}}$, this is a $p_{x}$ form vanishing along a nodal line (in the relative coordinate) parallel to the $x$ axis. Unfortunately, in contrast with the Laughlin case, squaring the determinant leads here to an "extended" $s$-wave form with a quadratic nodal line rather than the desired $d$ wave.

However, consider instead multiplying together two fermion determinants, each constructed by filling up a Fermi sea of momentum states, but with different Fermi surfaces. A wave function with $d_{x y}$ two-particle correlations can be constructed by choosing two elliptical Fermi surfaces, one with its long axis along the $x$ axis and the other rotated by $90^{\circ}$, as illustrated in Fig. 1,

$$
\Psi\left(\mathbf{r}_{1}, \mathbf{r}_{2}, \ldots, \mathbf{r}_{N}\right)=(\operatorname{det})_{x} \times(\operatorname{det})_{y} \quad\left(D_{x y} \text { type }\right),
$$

where the shorthands $(\operatorname{det})_{x}$ and $(\operatorname{det})_{y}$ represent the corresponding Slater determinants. In the limit of extreme eccentricity of the ellipses, the wave function will have the desired $d_{x y}$ form when two particles are brought close together $\left[\left(x_{i}-x_{j}\right)\left(y_{i}-y_{j}\right)\right]$. Away from this limit, the two nodal lines will not align precisely with the $x$ and $y$ axes, but upon taking one particle around the other, the wave function will exhibit the same sign structure as a $d_{x y}$ orbital, +-+- , changing sign twice. A picture of such sign structure as seen by a test particle is shown in Fig. 2.

Our principal thesis is that this wave function captures qualitative features of a different quantum liquid phase of bosons, which we call " $d$-wave correlated Bose liquid"
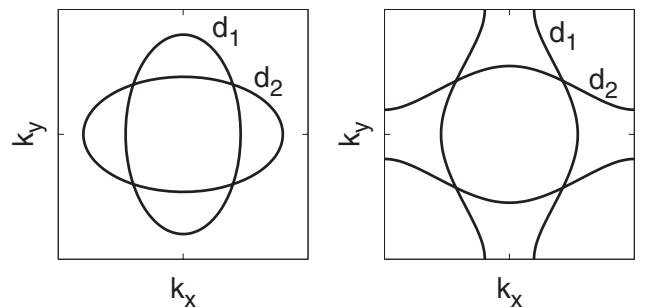

FIG. 1. DBL wave function Eq. (3) is obtained by multiplying two Slater determinants corresponding to distinct but symmetry related Fermi surfaces, which can be viewed as Fermi surfaces of $d_{1}$ and $d_{2}$ slave fermions. The left panel shows an example with closed elliptical Fermi surfaces, while the right panel is a case with open Fermi surfaces in the first Brillouin zone of the square lattice.

(DBL). However, a variational wave function does not provide a complete characterization of a quantum phase, and hence cannot be used to address its stability. As for the FQHE, a field theoretic approach, such as Chern-Simons gauge theory, is both desirable and ultimately necessary. Since we require time reversal invariance, Chern-Simons is inappropriate, but a tractable field theoretic framework for the DBL will, nevertheless, turn out to be a gauge theory.

Indeed, we follow closely the gauge theory approaches to spin liquids in quantum antiferromagnets, ${ }^{5,11}$ which after all are lattice bosonic systems. However, there are some notable and important differences for the DBL, which we will discuss below. The variational wave function in Eq. (3), being a product of two fermion determinants, naturally suggests expressing the boson creation operator as a product of two fermion operators,

$$
b^{\dagger}(\mathbf{r})=d_{1}^{\dagger}(\mathbf{r}) d_{2}^{\dagger}(\mathbf{r}),
$$

with the 2D position $\mathbf{r}$ either continuous or denoting the discrete sites of a square lattice. In a general case, this de-

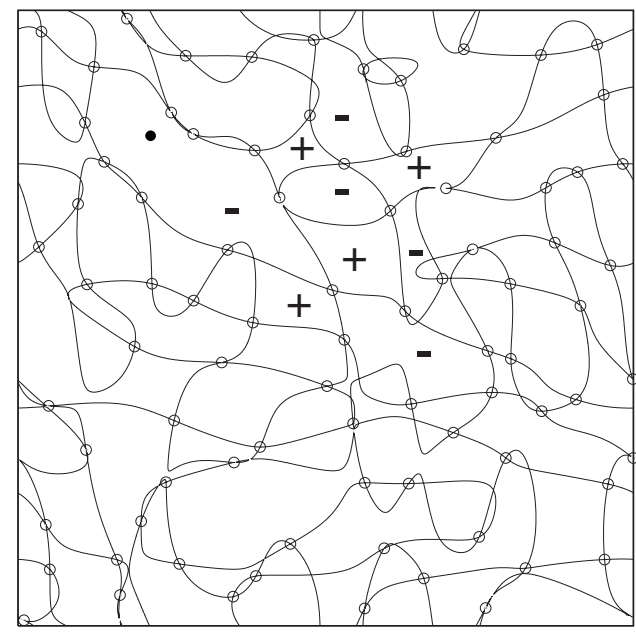

FIG. 2. "Nodal picture" of the continuum DBL wave function as probed by moving a single particle (whose initial location is marked by the filled circle) while keeping the rest of the particles fixed (open circles). The signs of the wave function are indicated in several places to bring out the +-+- pattern upon encircling a target particle. 
composition introduces a local $\mathrm{SU}(2)$ gauge redundancy wellknown in the slave-fermion treatments of the spin-1/2 antiferromagnet (see Ref. 11 for a recent comprehensive discussion). The $S$-type wave function corresponds to a socalled SU(2) liquid, while the DBL wave functions are U(1) liquids and require a $\mathrm{U}(1)$ gauge field which is minimally coupled to the two fermions with opposite gauge charges (the product $d_{1}^{\dagger} d_{2}^{\dagger}$ is then a gauge neutral composite, the physical boson).

Within slave-particle theory, a mean field state with a fixed background gauge "magnetic" flux is chosen. This choice is constrained by symmetry: All the physical symmetries of the boson model, even if not respected by the mean field Ansatz, must be present in the full gauge theory once gauge transformations are allowed. The collection of these symmetry transformations in the gauge theory is called the projective symmetry group (PSG). ${ }^{11}$ For lattice boson theories, many different mean field states with different PSG's are possible, while little is offered to guide which mean field state is the correct one.

In the continuum, the symmetries of the Euclidean plane together with time reversal invariance are more restrictive and we can only offer two PSG mean field Ansätze in this case. One is a mean field state with zero gauge flux and spherical Fermi seas for both fermions; this is an SU(2) Ansatz and corresponds to the $S$-type wave function considered earlier. A different U(1) Ansatz is obtained by considering $d_{1}$ and $d_{2}$ fermions, each moving in a uniform field but of opposite signs for the two. In this case, the boson wave function is schematically $\Psi=(\operatorname{det}) \times(\operatorname{det})^{*}$, e.g., $\Psi=|\operatorname{det} \mathbf{V}|^{2}$ $=\left|\Psi_{\nu=1 / 2}\right|$ to give a concrete example. This wave function is again non-negative and has off-diagonal quasi-long-range order. $^{12,13}$

For bosons moving in continuous space but with only the square lattice point group symmetry, we can offer only four PSG mean fields: all of them have zero flux, but differ in the way the $90^{\circ}$ rotation and mirror symmetries are realized. One example has both $d_{1}$ and $d_{2}$ Fermi surfaces individually respecting the symmetries but otherwise independent of each other. Two more examples are the already introduced $D_{x y}$ state [Eq. (3) and Fig. 1], with elliptical Fermi surfaces elongated along the $x$ and $y$ axes, and a similarly constructed $D_{x^{2}-y^{2}}$ state with ellipses along $\hat{\mathbf{x}} \pm \hat{\mathbf{y}}$. In both cases, the $d_{1}$ and $d_{2}$ Fermi surfaces are related by the $90^{\circ}$ rotation, but the two states differ in the way the mirror symmetries are realized. In the fourth example, each Fermi surface is invariant under the rotation but not under the mirrors, which instead are realized by interchanging the two fermions.

Each of the four presented Ansätze can be loosely called a $d$-wave Bose liquid as far as the nodal pictures like that in Fig. 2 are concerned; the gauge theory analysis would also be rather similar. From now on, we will focus on the $D_{x y}$ boson liquid. The associated mean field wave function once Gutzwiller projected into the physical Hilbert space, $d_{1}^{\dagger} d_{1}$ $=d_{2}^{\dagger} d_{2}$, is precisely the variational wave function in Eq. (3). A full incorporation of gauge fluctuations about the mean field performs, in principle, this projection exactly. However, in practice, the gauge theory approach allows incorporation of slowly varying gauge fluxes and is different in this respect from the wave function. Provided the gauge theory is not in a confined state and no physical symmetries are broken, an exact description of the putative DBL phase is thereby obtained. Herein, we introduce a large- $N$ generalization of the gauge theory, which allows a controlled and systematic treatment of the gauge fluctuations order by order in powers of $1 / N$. We can then perturbatively extract physical properties of the DBL phase. Whether the DBL phase survives down to $N=1$ is not something that we can reliably address.

In describing the $D_{x y}$ liquid phase for lattice bosons, there is considerable freedom when choosing the precise form of the slave-fermion hopping in the mean field Hamiltonian. The simplest choice is near-neighbor hopping with different amplitudes in the $x$ and $y$ directions. However, even with this restriction, there are two different possible Fermi surface topologies, closed or open, as depicted in Fig. 1. The Fermi surface topology has rather dramatic consequences for the nature of the associated $d$-wave Bose fluid. When the Fermi surfaces are open and have no parallel Fermi surface tangents, the resulting phase has a quasilocal character-the boson Green's function is found to fall off exponentially in space. This is a distinct quantum phase which we refer to as a $d$-wave local Bose liquid (DLBL). The DLBL is intrinsically very stable against gauge fluctuations and we are fairly confident that, when present, it will be a stable quantum phase. The effect of gauge fluctuations on the DBL phase, however, is more subtle. Within our systematic large- $N$ approach, we do find a regime where the DBL is stable, but it is less clear that this will survive down to $N=1$. The Gutzwiller wave function does appear to describe a DBL phase with properties that are consistent with those inferred from the gauge theory. This provides some support for the stability of the DBL phase.

Together the gauge theory and the variational wave function provide a consistent and rather complete picture of both the DBL and the DLBL. Here, we briefly highlight some of the important characteristics. In the DBL, the single-particle boson Green's function, $G_{b}(\mathbf{r}, \tau) \equiv\left\langle b^{\dagger}(\mathbf{r}, \tau) b(\mathbf{0}, 0)\right\rangle$, decays as an oscillatory power law at equal times,

$$
G_{b}(\mathbf{r}, 0) \sim \frac{\cos \left[\left(\mathbf{k}_{F_{1}}-\mathbf{k}_{F_{2}}\right) \cdot \mathbf{r}\right]}{|\mathbf{r}|^{4-\eta}}+\frac{\cos \left[\left(\mathbf{k}_{F_{1}}+\mathbf{k}_{F_{2}}\right) \cdot \mathbf{r}-3 \pi / 2\right]}{|\mathbf{r}|^{4}},
$$

where the two wave vectors $\mathbf{k}_{F_{1,2}}$ depend on the observation direction $\hat{\mathbf{r}}$, as does the anomalous exponent $\eta>0$. As the direction of $\hat{\mathbf{r}}$ is rotated in real space, the wave vectors $\mathbf{k}_{F_{1,2}}$ and $\mathbf{k}_{F_{1}} \pm \mathbf{k}_{F_{2}}$ trace out closed momentum space curves, and for the DBL in the continuum, these curves will have the topology of a circle.

If one measures the boson Green's function and finds it to be of the above form, it is natural to refer to the $\mathbf{k}_{F_{1}} \pm \mathbf{k}_{F_{2}}$ as "Bose surfaces." Within the gauge theory description, the origin of these singular surfaces can be traced to the Fermi surfaces of the constituent fermions. Specifically, $\mathbf{k}_{F_{1,2}}$ are locations on the two Fermi surfaces where the surface normals are along the observation direction $\hat{\mathbf{r}}$, and the momentum space areas enclosed by each of these surfaces will equal $(2 \pi)^{2} \rho_{b}$. Note that the $\mathbf{k}_{F_{1,2}}$ surfaces can be uniquely recon- 
structed from the measured Bose surfaces $\mathbf{k}_{F_{1}} \pm \mathbf{k}_{F_{2}}$. In this sense, the Bose surfaces in the DBL contain information analogous to Luttinger's Fermi surface volume theorem in the Fermi liquid. The Bose surface can also be extracted directly from the DBL wave function by using variational Monte Carlo to compute the equal-time boson Green's function, and can also be indirectly inferred by using the shape of the two Fermi surfaces input into the (det) $)_{x}$ and (det) $)_{y}$ factors. It is both reassuring and quite remarkable that these two coincide.

The spatially local Green's function, $G_{b}(\mathbf{0}, \tau)$, in the DBL falls off in time as a power law, which is at least as slow as $1 / \tau^{2}$, corresponding to the local boson tunneling density of states $A_{b}(E) \sim E$. These are mean field results obtained by combining two fermions each with a finite density of states, but we suspect the time decay may actually be slower upon including gauge fluctuations.

The behavior of the boson Green's function in the DLBL is quite different. The time decay at $\mathbf{r}=0$ is a power law, $G_{b}(0, \tau) \sim \tau^{-2}$, which is an exact result insensitive to lattice scale details. However, the equal-time Green's function falls off exponentially in space in the DLBL, $G_{b}(\mathbf{r}, 0) \sim \exp (-r / \xi)$. Despite this, a two boson box correlator,

$$
\mathcal{B}_{b}(x) \equiv\left\langle b^{\dagger}(0,0) b^{\dagger}(x, x) b(x, 0) b(0, x)\right\rangle,
$$

falls off as a (nonoscillatory) power law at large distances in the DLBL, $\mathcal{B}(x) \sim-x^{-8}$, with both the sign (negative) and the exponent being universal and insensitive to lattice scale physics. Paradoxically, this seems to imply that a pair of bosons injected into the DLBL on opposite corners of a square box can move more readily than a single injected boson. However, in a strongly interacting quantum state such a single-particle interpretation can be misleading-the dynamics of an injected boson is not that of a weakly interacting quasiparticle.

In both the DBL and the DLBL, the density-density correlator $D_{b}(\mathbf{r}) \equiv\left\langle\hat{\rho}_{b}(\mathbf{r}) \hat{\rho}_{b}(\mathbf{0})\right\rangle$ behaves at large distances as

$$
D_{b}(\mathbf{r}) \sim-\sum_{\alpha=1,2} \frac{\cos \left[2 \mathbf{k}_{F_{\alpha}} \cdot \mathbf{r}-3 \pi / 2\right]}{|\mathbf{r}|^{4-\gamma_{\alpha}}}+\frac{1}{|\mathbf{r}|^{4}},
$$

where, again, both the wave vectors $\mathbf{k}_{F_{1,2}}$ and the anomalous exponent $\gamma_{\alpha}$ depend on the observation direction $\hat{\mathbf{r}}$. Measurement of such correlation can be used to extract $\mathbf{k}_{F_{1,2}}$ in the DLBL, which is not accessible from the boson Green's function here. Upon rotating the unit vector $\hat{\mathbf{r}}$ in real space, $\mathbf{k}_{F_{1,2}}$ will again trace out the Fermi surfaces of the underlying fermions which for the DLBL will be open. Both the DBL and the DLBL are conductors with a resistance varying with temperature as $R \sim T^{4 / 3}$. This is particularly striking for the DLBL, where the boson Green's function is short ranged. Evidently, it is not possible to understand the metallic transport in terms of the motion of weakly interacting bosons. In a sense, it is the fermionic constituents which transport the charge, but even this is not quite right since the $d_{1}, d_{2}$ fermions do not exist as well defined quasiparticle excitations, being strongly scattered by the gauge fluctuations.
The lattice gauge theory formulation can be used to motivate a lattice boson Hamiltonian, which might plausibly have the DBL/DLBL as a ground state. The simplest such model consists of a near-neighbor boson hopping term supplemented by a four-site "ring" exchange term:

$$
\begin{gathered}
H=H_{J}+H_{4}, \\
H_{J}=-J \sum_{\mathbf{r} ; \hat{\mu}=\hat{\mathbf{x}} \hat{\mathbf{y}}}\left(b_{\mathbf{r}}^{\dagger} b_{\mathbf{r}+\hat{\mu}}+\text { H.c. }\right), \\
H_{4}=K_{4} \sum_{\mathbf{r}}\left(b_{\mathbf{r}}^{\dagger} b_{\mathbf{r}+\hat{\mathbf{x}}} b_{\mathbf{r}+\hat{\mathbf{x}}+\hat{\mathbf{y}}}^{\dagger} b_{\mathbf{r}+\hat{\mathbf{y}}}+\text { H.c. }\right),
\end{gathered}
$$

with $J, K_{4} \geqslant 0$. This model is fully specified by two dimensionless numbers, the ratio $J / K$ and the boson filling $0 \leqslant \rho_{b}$ $\leqslant 1$. This ring Hamiltonian with $J>0$ and $K_{4}<0$ was introduced and studied by Paramekanti et al. ${ }^{14}$ and later studied extensively with quantum Monte Carlo by Sandvik et al. ${ }^{15}$ Melko et al.,${ }^{16}$ and Rousseau et al. ${ }^{17}$ There is no sign problem in this regime, and it was possible to access large system sizes and low temperatures. However, for $J>0$ and $K_{4}>0$, there is a sign problem since the Hamiltonian does not satisfy the Marshall sign conditions, and one expects the ground state wave function to take both positive and negative values. For this ring model, we have evaluated the variational energetics for the DBL/DLBL wave functions and compared these to the energy of a superfluid wave function of the usual Jastrow form. Within this necessarily limited energetics study, we do find a region of parameter space with small $J / K_{4}$ and near half filling, where the DLBL wave function has the lowest energy. In view of its quasilocal character, we suspect that density matrix renormalization group (DMRG) studies could be fruitful in helping establish whether or not the DLBL is present in the phase diagram of this (or related) ring Hamiltonian.

The paper is organized as follows. In Sec. II, we discuss in more detail the wave function motivation for considering the DBL states. In Sec. III, we introduce the lattice gauge theory description and study properties of the DBL state in the mean field theory that ignores gauge fluctuations. This provides an initial guide to the singular (Bose) surfaces, and is followed in Sec. IV with numerical characterization of the properties of the actual DBL wave functions. In Sec. V, we consider the full gauge theory description, focusing on the effects of the gauge fluctuations on the singularities across the Bose surfaces, and, in particular, obtain the long-distance properties such as Eqs. (5) and (7) to order $1 / N$ in our systematic large- $N$ approach. In Sec. VI, we address the issue of the stability of the DBL by considering a putative fixed-point theory. We conclude in Sec. VIII with a discussion of physical properties and possible future directions.

\section{BOSON WAVE FUNCTIONS AND NODES}

In this section, we expand on the initial motivation for considering the DBL states as a way to perform (a kind of) flux-attachment transformation in a time reversal invariant manner. We also discuss the nodal structure of the bosonic 
wave functions, providing some justification for the qualifier " $d$ wave" in the suggested wave function names in this work. To this end, we consider a "relative single-particle wave function"-more precisely, a cross section of the many-body wave function-defined as follows. Fixing the positions of all the particles except one, we define a function which depends explicitly on the coordinates of one "test" particle and implicitly on the coordinates of all the other particles,

$$
\Phi_{\mathbf{r}_{2}, \ldots, \mathbf{r}_{N}}(\mathbf{r}) \equiv \Psi\left(\mathbf{r}, \mathbf{r}_{2}, \ldots, \mathbf{r}_{N}\right) .
$$

For notational ease, we will henceforth drop the implicit dependence on the $2(N-1)$ spatial coordinates and just use the notation $\Phi_{b}(\mathbf{r})$.

\section{A. Laughlin $\nu=1 / 2$ revisited}

Consider the Laughlin wave function for bosons in a halffilled Landau level [Eq. (1)]. In this case, the relative singleparticle wave function $\Phi(z)$ is complex and has double strength zeroes at the positions of all the other $N-1$ particles. Upon close approach to a specific particle, say $z_{i}$, the function $\Phi(z) \sim\left(z-z_{i}\right)^{2}$ is of a $d+i d$ form. It is in this sense that the $\nu=1 / 2$ Laughlin state can be viewed as a $d$-wave fluid: All pairs of particles, upon close approach in real space, are in a two-body $d+i d$ state. Our goal is to construct a time reversal invariant analog of the $\nu=1 / 2$ Laughlin state, in which particle pairs are similarly in a relative $d_{x y}$ or $d_{x^{2}-y^{2}}$ state.

In thinking about Laughlin states, it has been particularly instructive to view them in terms of composite particles created by "flux attachment." For example, if one reexpresses the $\nu=1 / 2$ Laughlin state as

$$
\Psi_{\nu=1 / 2}=\prod_{i<j}\left(z_{i}-z_{j}\right) \Psi_{c f}
$$

the "composite fermion" wave function is simply a (Vandermonde) determinant, det $\mathbf{V}$, of filled Landau level orbitals,

$$
\Psi_{c f}=\operatorname{det} \mathbf{V}=\prod_{i<j}\left(z_{i}-z_{j}\right),
$$

with $V_{i j}=\left(z_{i}\right)^{j}$. In a second quantized framework, flux attachment can be achieved by introducing a Chern-Simons gauge field. ${ }^{18}$ Chern-Simons gauge theory has been a useful tool in describing the full Haldane-Halperin hierarchy of fractional quantum Hall states, encoding the fractional charge and statistics of the quasiparticles as well as the structure of the edge excitations. ${ }^{19,20}$ However, in discussing bosons in a time reversal invariant setting (zero magnetic field), fluxattachment techniques are problematic. For instance, the usual flux smearing mean field approximation is likely to inadvertently break time reversal invariance.

In seeking to avoid Chern-Simons theory, it is worth noting that the $\nu=1 / 2$ state is a perfect square-a square of the Vandermonde determinant,

$$
\Psi_{\nu=1 / 2}=(\operatorname{det} \mathbf{V})^{2} .
$$

This suggests that the $\nu=1 / 2$ Laughlin state can be fruitfully described within a slave-particle framework. ${ }^{21}$ Consider de- composing the boson creation operator as a product of two fermions, $b^{\dagger}(\mathbf{r})=d_{1}^{\dagger}(\mathbf{r}) d_{2}^{\dagger}(\mathbf{r})$. As discussed in Sec. I, there is a local gauge redundancy, and a correct treatment requires the presence of a gauge field minimally coupled to both fermions. In the slave-particle mean field approach to the $\nu=1 / 2$ boson problem, one simply drops the gauge field, obtaining a problem of two fermion flavors each moving in a magnetic field. If the electrical charge of the boson is divided equally, each fermion flavor is effectively in a full Landau level. The mean field (MF) wave function is

$$
\Psi_{\nu=1 / 2}^{M F}=\prod_{i<j}\left(z_{i}^{(1)}-z_{j}^{(1)}\right) \prod_{i<j}\left(z_{i}^{(2)}-z_{j}^{(2)}\right) .
$$

To obtain a wave function for the bosons, it is necessary to project into the physical Hilbert space, and this is achieved here by simply setting $z_{i}^{(1)}=z_{i}^{(2)}=z_{i}$. One thereby recovers the Laughlin state as a square of the Vandermonde determinant [Eq. (14)].

\section{B. Time reversal invariant wave functions}

Within a first quantized framework, the relative simplicity of a time reversal invariant bosonic superfluid as compared to the Laughlin state is the nodelessness of the ground state wave function. As Feynman argued many years ago, for nonrelativistic bosons moving in the continuum with an interaction only depending on the particle positions, the kinetic energy of any bosonic wave function which has sign changes could be reduced by making all the signs positive while keeping the magnitude of the wave function fixed to leave the potential energy unchanged. The ground state wave function should, thus, be everywhere non-negative. For the noninteracting Bose gas, the ground state wave function is just $\Psi=1$, but in the presence of interactions, a popular variational wave function is of the Jastrow form, ${ }^{13}$

$$
\Psi_{\text {Jastrow }}\left(\mathbf{r}_{1}, \mathbf{r}_{2}, \ldots, \mathbf{r}_{N}\right) \propto \exp -\sum_{i<j} u\left(\mathbf{r}_{i}-\mathbf{r}_{j}\right),
$$

with variational freedom in the two-particle pseudopotential $u\left(\mathbf{r}_{i}-\mathbf{r}_{j}\right)$, which is usually taken to approach zero as $1 /|\mathbf{r}|^{p}$ at large separations.

As in the case with a magnetic field present, we again consider the relative single-particle wave function. For a Bose condensed superfluid in a time reversal invariant system, $\Phi_{b}(\mathbf{r})$ can be taken as real and is everywhere nonnegative. If there are repulsive interactions between the bosons in the superfluid, the amplitude of $\Phi_{b}(\mathbf{r})$ will be reduced when the test particle is taken nearby another particle, which is implemented by the Jastrow pseudopotential in Eq. (16). However, the sign of $\Phi_{b}(\mathbf{r})$ will remain positive, so in some sense all of the particle pairs are in a relative $S$-type state. In the special case of a hard-core interaction, $\Phi_{b}(\mathbf{r})$ $\rightarrow 0$ for $\mathbf{r} \rightarrow \mathbf{r}_{i}$, so one can then view this as an extended $S$-type wave function.

Motivated by the preceding discussion of the $\nu=1 / 2$ Laughlin state, in Eq. (2) we introduced a simple time reversal invariant wave function for hard-core bosons, which is the square of a determinant for free fermions filling a Fermi sea. By construction, this wave function is non-negative. 
Moreover, the wave function will have zeroes which coincide with the nodes of the filled Fermi sea (FS), $\Psi_{F S}\left(\mathbf{r}_{1}, \mathbf{r}_{2}, \ldots, \mathbf{r}_{N}\right)=\operatorname{det} e^{i \mathbf{k}_{i} \cdot \mathbf{r}_{j}}$. With time reversal invariance, a relative single fermion wave function, $\Phi_{f}(\mathbf{r})$ $\equiv \Psi_{F S}\left(\mathbf{r}, \mathbf{r}_{2}, \ldots, \mathbf{r}_{N}\right)$, can be taken as real. As a result, the nodal structure of $\Phi_{f}(\mathbf{r})$ will be qualitatively different than for the filled Landau level state, vanishing along nodal lines rather than at isolated points. These nodal lines will pass through the positions of all of the other fermions. ${ }^{10}$ Upon taking the test particle across a nodal line, the function $\Phi_{f}(\mathbf{r})$ changes sign, vanishing linearly upon approaching the nodal line. If one takes the position of the test particle close to another particle, $\Phi_{f}(\mathbf{r})$ will have a $p$-wave character-in particular, a $p_{x}$ form if we define the $x$ axis as being parallel to the nodal line.

Despite the $p$-wave character of the free fermion determinant, the determinant-squared wave function will not have a $d$-wave character. Rather, the relative single boson wave function will vanish quadratically upon crossing the nodal lines of the fermion determinant. When the test particle is taken near another particle $\mathbf{r}_{i}$, the function vanishes quadratically $\left[\Phi_{b}(\mathbf{r}) \sim\left(x-x_{i}\right)^{2}\right]$. The "pair" wave function is thus of an extended $S$-type form, vanishing along the residual nodal line.

Let us now consider the DBL many-body wave function Eq. (3), which is the product of two different Slater determinants of fermions that fill elliptical Fermi surfaces as shown in Fig. 1. The nodal structure is revealed by exploring $\Phi_{b}(\mathbf{r})$. This function will have two sets of nodal lines, one from each of the determinants. Due to the elliptical nature of the respective Fermi surfaces, the two sets of lines, both of which pass through all of the particles, will generally not coincide with one another. Indeed, the fermion determinant coming from an elliptical Fermi surface will have nodal lines running preferentially perpendicular to the long axis of the ellipse. Focusing on the behavior of $\Phi_{b}(\mathbf{r})$ near a target particle $\mathbf{r}_{i}$, one anticipates a behavior of the $d_{x y}$ form, $\Phi_{b}(\mathbf{r})$ $\sim\left(x-x_{i}\right)\left(y-y_{i}\right)$. Here, we have assumed that the two nodal lines actually coincide with the $x$ and $y$ axes. In general, for a typical configuration of fixed particle coordinates and a given target particle, this will not precisely be the case. More generically, the two nodal lines will intersect a particle at two angles which are not aligned with the axes. However, the sign of the relative wave function when the test particle encircles the target particle will still behave as +-+- , the same sign structure as a $D_{x y}$ or $D_{x^{2}-y^{2}}$ orbital. This is illustrated in Fig. 2.

Since the DBL many-body wave function is not nodeless, it cannot be the ground state of a continuum Hamiltonian of bosons. If we put the coordinates on the sites of a $2 \mathrm{D}$ square lattice, the ground state wave function is only assured to be non-negative if the sign and form of the hopping matrix in the lattice tight binding Hamiltonian are such that they satisfy the Marshall sign conditions - the requirement that a choice of gauge is possible to make all of the off-diagonal matrix elements negative. In Sec. VII, we consider a particular Marshall sign violating lattice boson Hamiltonian which might exhibit a ground state of the proposed $d$-wave form.

\section{Precedents of (det) ${ }_{1} \times(\text { det })_{2}$ wave functions for spin liquids}

Before focusing solely on the DBL states, we want to mention that our construction of time reversal invariant bosonic wave functions as a product of two distinct determinants has nice precedents in the studies of spin liquids on the triangular lattice. One can view the triangular Heisenberg antiferromagnet as a system of hard-core bosons at half filling in the background field of flux $\pi$ through each triangle. Kalmeyer and Laughlin ${ }^{22}$ proposed to view this in the continuum, obtaining a boson system at $\nu=1 / 2$, and their chiral spin liquid wave function is precisely the lattice analog of the $\nu=1 / 2$ state [Eq. (1)]. Alternatively, using a slavefermion approach, ${ }^{23,24} b^{\dagger}=d_{1}^{\dagger} d_{2}^{\dagger}$, we divide the boson charge equally between the two fermions, so each sees, on average, a flux of $\pi / 2$ per triangle; the mean field where the $d_{1}$ and $d_{2}$ see the same static flux of $\pi / 2$ per triangle gives a filled Landau level for each fermion and reproduces precisely the Laughlin-Kalmeyer chiral spin liquid.

However, we can be more creative about the fluxes seen by the slave fermions while maintaining the average of $\pi / 2$ flux per triangle. One example is to take different flux patterns for the two species as follows: For the $d_{1}$ fermions, put 0 flux through all up-pointing triangles and $\pi$ flux through all down-pointing triangles, while for the $d_{2}$ fermions, interchange the locations of the 0 and $\pi$ fluxes. This state is, in fact, identical to the so-called $U 1 B \tau^{1} \tau_{-}^{0} \tau_{+}^{1}$ spin liquid found in Ref. 25. It is a time reversal invariant gapless algebraic spin liquid (ASL) with Dirac nodes in the spinon spectrum; it has very good energetics for the nearest neighbor triangular antiferromagnet, starting from the isotropic lattice and all the way to the limit of weakly coupled chains (e.g., Ref. 26 found a different gauge-equivalent formulation of this state without realizing its ASL character).

Another example is obtained by taking yet different flux patterns: Select one lattice direction-chain direction in the anisotropic lattice case. For the $d_{1}$ fermions, put 0 flux through triangles siding even chains and $\pi$ flux through triangles siding odd chains, while for the $d_{2}$ fermions, interchange the locations of the 0 and $\pi$ fluxes. This is again a time reversal invariant ASL and is listed as $U 1 C \tau_{+}^{0} \tau_{-}^{0} \tau^{1}$ in Ref. 25. Unlike the $U 1 B$ state, there is no isotropic $U 1 C$ liquid, but the energetics performance of $U 1 C$ and $U 1 B$ spin liquids is almost indistinguishable for weakly coupled chains and matches that of competing magnetically ordered states; in this context, the time reversal invariant $U 1 B$ and $U 1 C$ liquids are significantly better than the chiral LaughlinKalmeyer variant.

Our construction of the DBL states differs in that we do not require any special filling for the bosons and there is no special Heisenberg spin symmetry. Neither of these special conditions of the spin model setting are needed for the construction and subsequent gauge theory analysis to go through. Given the growing belief $f^{11,27,28}$ that critical spin liquids do exist, we do not see any reasons why the situation should be any different for our boson liquids at arbitrary incommensurate densities. In our construction, such liquids will generically have some underlying partially filled bands and, therefore, Fermi surfaces of slave fermions. Of course, whether a particular state is realized in a given model re- 
quires a detailed case by case study, and in Sec. VII, we suggest some frustrated boson models that may stabilize the DBL phase. Our primary goal in Secs. III-VI will be to characterize the DBL states without worrying where to find them.

\section{MEAN FIELD THEORY FOR THE $\boldsymbol{d}$-WAVE BOSE LIQUID}

\section{A. Gauge theory formulation}

We next consider the challenge of constructing a field theory which can access such a $D_{x y}$-Bose liquid (DBL) state. Due to our inability to implement flux attachment in a tractable time reversal invariant manner, we follow instead the slave-particle approach. As above, we decompose the hardcore lattice boson as a product of two fermions, $b^{\dagger}=d_{1}^{\dagger} d_{2}^{\dagger}$. Consider then a lattice U(1) gauge theory on the square lattice in terms of these slave particles:

$$
H_{\mathrm{U}(1)}=H_{t}+H_{a},
$$

with the fermion hopping Hamiltonian of the form

$$
\begin{aligned}
H_{t}= & -\sum_{\mathbf{r}}\left[t_{\|} e^{i a_{x}(\mathbf{r})} d_{1}^{\dagger}(\mathbf{r}) d_{1}(\mathbf{r}+\hat{\mathbf{x}})+t_{\perp} e^{i a_{y}(\mathbf{r})} d_{1}^{\dagger}(\mathbf{r})\right. \\
& \left.\times d_{1}(\mathbf{r}+\hat{\mathbf{y}})+\text { H.c. }\right]-\sum_{\mathbf{r}}\left[t_{\perp} e^{-i a_{x}(\mathbf{r})} d_{2}^{\dagger}(\mathbf{r}) d_{2}(\mathbf{r}+\hat{\mathbf{x}})\right. \\
& \left.+t_{\|} e^{-i a_{y}(\mathbf{r})} d_{2}^{\dagger}(\mathbf{r}) d_{2}(\mathbf{r}+\hat{\mathbf{y}})+\text { H.c. }\right] .
\end{aligned}
$$

The $d_{1}$ fermion hopping amplitudes in the $\hat{\mathbf{x}}$ and $\hat{\mathbf{y}}$ directions are $t_{\|}$and $t_{\perp}$, respectively, while the two amplitudes are interchanged for the $d_{2}$ fermions. In the following, we take $t_{\|}$ $\geqslant t_{\perp}$ for concreteness. Note also that the two fermion species carry opposite gauge charges. The gauge field Hamiltonian is simply

$$
H_{a}=h \sum_{\mathbf{r}} \sum_{\mu=x, y} e_{\mu}^{2}(\mathbf{r})-K \sum_{\mathbf{r}} \cos \left[(\nabla \times a)_{\mathbf{r}}\right],
$$

where the lattice magnetic field is

$$
(\nabla \times a)_{\mathbf{r}}=a_{x}(\mathbf{r})+a_{y}(\mathbf{r}+\hat{\mathbf{x}})-a_{x}(\mathbf{r}+\hat{\mathbf{y}})-a_{y}(\mathbf{r}) .
$$

The integer-valued "electric" field $e_{\mu}(\mathbf{r})$ is canonically conjugate to the compact gauge field $a_{\mu}(\mathbf{r})$ on the same link. The above Hamiltonian is supplemented by a gauge constraint on the physical states, which must satisfy Gauss law,

$$
(\nabla \cdot e)_{\mathbf{r}}=d_{1}^{\dagger}(\mathbf{r}) d_{1}(\mathbf{r})-d_{2}^{\dagger}(\mathbf{r}) d_{2}(\mathbf{r}),
$$

with

$$
(\nabla \cdot e)_{\mathbf{r}}=e_{x}(\mathbf{r})-e_{x}(\mathbf{r}-\hat{\mathbf{x}})+e_{y}(\mathbf{r})-e_{y}(\mathbf{r}-\hat{\mathbf{y}}) .
$$

In the limit $h \gg K, t_{\|}, t_{\perp}$, the electric field vanishes and Gauss law reduces to $d_{1}^{\dagger} d_{1}=d_{2}^{\dagger} d_{2}$, which projects back into the physical boson Hilbert space. In this strong coupling limit, it is possible to perturbatively eliminate the gauge field to obtain a Hamiltonian for hard core bosons hopping on the square lattice with additional ring exchange terms. We pursue this in Sec. VII, where we compare the energetics of the DBL, wave function with other states. Here, we instead focus on the weak coupling limit, $K \gg h$, which suppresses the magnetic flux. The usual slave-particle mean field treatment corresponds to simply setting $(\nabla \times a)_{\mathbf{r}}$ equal to a constant. The simplest mean field state, and the one which should correspond to the wave functions in the previous section, is with zero flux through all plaquettes. The corresponding mean field Hamiltonian describes noninteracting slave fermions. Each species has anisotropic near-neighbor hopping amplitudes, but the two are related under the $90^{\circ}$ rotation, thus producing a boson liquid that respects the symmetries of the square lattice.

\section{B. Mean field results for the DBL}

In order to focus on the effects of the underlying Fermi surfaces without the complications of lattice physics such as Brillouin zone folding, we first consider fermions in the 2D continuum with anisotropic effective masses. The resulting elliptical Fermi surfaces are

$$
\begin{aligned}
& d_{1}:\left(w k_{x}\right)^{2}+\left(k_{y} / w\right)^{2}=k_{F}^{2}, \\
& d_{2}:\left(k_{x} / w\right)^{2}+\left(w k_{y}\right)^{2}=k_{F}^{2},
\end{aligned}
$$

where the parameter $w \geqslant 1$ characterizes the degree of eccentricity (the conventional eccentricity of the ellipses is given by $\epsilon=\sqrt{1-1 / w^{2}}$ ). In the $d$-wave Bose liquid, $w$ signifies the mismatch between the two Fermi surfaces, and we will refer to this measure as " $d$-eccentricity." We study equal-time correlation functions since these can be compared directly with the properties of the wave functions, which is done in Sec. IV; we also consider temporal dependencies within the mean field as a measure of spectral properties. It is useful to have in mind that much of the following analysis of long-distance properties needs only the knowledge of relevant Fermi surface patches and not of the full surfaces.

Consider first the one-particle off-diagonal density matrix (or Green's function) for the boson, $G_{b}(\mathbf{r}, \tau)$ $=\left\langle b^{\dagger}(\mathbf{r}, \tau) b(\mathbf{0}, \tau)\right\rangle$, with $G_{b}(\mathbf{0}, 0)=\bar{\rho}$ - the average boson density. It will also be of interest to consider the momentum occupation probability,

$$
\left\langle n_{\mathbf{k}}\right\rangle=\left\langle b_{\mathbf{k}}^{\dagger} b_{\mathbf{k}}\right\rangle=\int d \mathbf{r} G_{b}(\mathbf{r}) e^{-i \mathbf{k} \cdot \mathbf{r}} .
$$

Within the mean field theory, the natural approximation for the order parameter correlation is

$$
G_{b}^{M F}(\mathbf{r}, \tau)=G_{d_{1}}^{M F}(\mathbf{r}, \tau) G_{d_{2}}^{M F}(\mathbf{r}, \tau) / \bar{\rho},
$$

where $G_{d_{\alpha}}^{M F}$ are the mean field (bare) fermion Green's functions. This approximation satisfies $G_{b}(\mathbf{0})=G_{d_{\alpha}}(\mathbf{0})=\bar{\rho}$. Here and below, the imaginary time $\tau$ is understood to be zero if not explicitly present.

The fermion Green's functions are readily calculated. Thus, at long distances $r \gg k_{F}^{-1}$, the main contribution to $G_{d_{\alpha}}^{M F}(\mathbf{r})$ comes from the Fermi surface patches where the group velocity is parallel or antiparallel to the observation direction $\hat{\mathbf{r}}=\mathbf{r} /|\mathbf{r}|$. With inversion symmetry, we can denote the corresponding patch locations as $\pm \mathbf{k}_{F_{\alpha}}$ and the Fermi surface curvature as $c_{\alpha}$, and obtain 

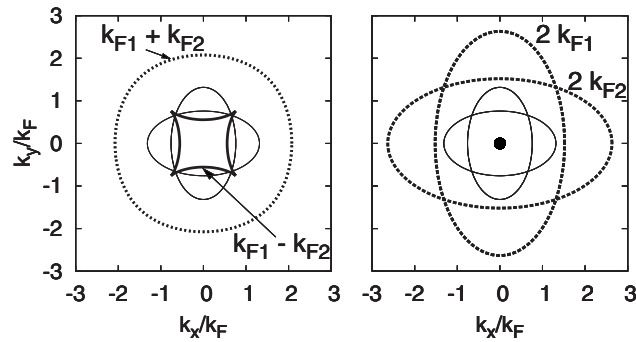

FIG. 3. Singular lines in the momentum space for the boson Green's function (left) and the density correlation (right). In each panel, the thin lines show the elliptical $d_{1}$ and $d_{2}$ Fermi surfaces for the parameter $w^{4}=3$ [cf. Eq. (23)]. In the left panel, the $\mathbf{k}_{F_{1}} \pm \mathbf{k}_{F_{2}}$ loci are constructed by considering a pair of $d_{1}$ and $d_{2}$ fermions with parallel $(+$ sign$)$ or antiparallel $(-\operatorname{sign})$ group velocities. The axes are scaled by an "average" $k_{F}$ that would be obtained if the Fermi surfaces were circular for the same particle density.

$$
G_{d_{\alpha}}^{M F}(\mathbf{r}) \approx \frac{1}{2^{1 / 2} \pi^{3 / 2}} \frac{\cos \left(\mathbf{k}_{F_{\alpha}} \cdot \mathbf{r}-3 \pi / 4\right)}{c_{\alpha}^{1 / 2}|\mathbf{r}|^{3 / 2}}
$$

It is important to remember that $\mathbf{k}_{F_{1}}, \mathbf{k}_{F_{2}}$ and $c_{1}, c_{2}$ depend implicitly on the direction $\hat{\mathbf{r}}$ and are different, in general, when the $d_{1}$ and $d_{2}$ Fermi surfaces do not coincide.

For the equal-time boson Green's function, we thus get

$$
G_{b}^{M F}(\mathbf{r}) \sim \frac{\cos \left[\left(\mathbf{k}_{F_{1}}-\mathbf{k}_{F_{2}}\right) \cdot \mathbf{r}\right]}{c_{1}^{1 / 2} c_{2}^{1 / 2}|\mathbf{r}|^{3}}+\frac{\cos \left[\left(\mathbf{k}_{F_{1}}+\mathbf{k}_{F_{2}}\right) \cdot \mathbf{r}-3 \pi / 2\right]}{c_{1}^{1 / 2} c_{2}^{1 / 2}|\mathbf{r}|^{3}},
$$

which decays algebraically while oscillating with $\hat{\mathbf{r}}$-direction-dependent wave vectors $\mathbf{k}_{F_{1}}+\mathbf{k}_{F_{2}}$ and $\mathbf{k}_{F_{1}}-\mathbf{k}_{F_{2}}$. Such wave vectors, which are constructed by considering patches on the two Fermi surfaces that are parallel to each other, will span some new loci in the momentum space as illustrated in Fig. 3. The above large-distance behavior corresponds to singularities $n_{\mathbf{k}} \sim|\delta k|^{3 / 2}$ across these lines. In the zero eccentricity limit (i.e., when $w=1$ ), the two Fermi surfaces coincide, giving

$$
G_{b}^{M F}(r) \sim \frac{1+\cos \left[2 k_{F} r-3 \pi / 2\right]}{r^{3}} \quad(\text { S type }) .
$$

Compared to the general case with $w>1$, the $\mathbf{k}_{F_{1}}-\mathbf{k}_{F_{2}}$ locus shrinks here to zero momentum, while the singularity in $n_{\mathbf{k}}$ is hardened to $|k|$.

As we will show in Sec. V, the power-law decay of the mean field boson Green's function survives in the presence of gauge fluctuations (but with modified exponents). The algebraic decay is a consequence (and a measurable indication) of the "criticality" of the DBL and its gapless excitations. Of course, by the very construction, we expect many gapless excitations because of the underlying Fermi surfaces. Some measure of the low-energy spectrum is contained in the time dependence of the local boson Green's function,

$$
G_{b}^{M F}(\mathbf{0}, \tau)=\frac{\nu_{0}^{2}}{\tau^{2}},
$$

where $\nu_{0}$ is the density of states at the Fermi energy for each species. The corresponding local boson spectral function is

$$
A_{b}^{M F}(\mathbf{r}=\mathbf{0}, E)=\int_{\mathbf{k}} A_{b}^{M F}(\mathbf{k}, E)=\nu_{0}^{2} E .
$$

In the discussion of the DBL phase on the lattice in Sec. III C, we will encounter a situation where the boson Green's function decays exponentially in space because of the topology of the Fermi surfaces, while the above spectral signature of the low-energy excitations depends only on the density of states, which is a property of the entire Fermi surface, and is insensitive to the topology otherwise.

The most natural instability of the DBL is toward a superfluid state. As we will discuss in Sec. VI, in the limit of vanishing $d$ eccentricity $(w=1)$, the resulting $S$-type Bose liquid phase obtained in mean field theory is, in the presence of gauge fluctuations, most probably unstable to superfluidity. Moreover, our Sec. IV analysis of the determinantsquared wave function appropriate to the $S$-Bose liquid is consistent with off-diagonal long-range order. These results strongly suggest that the mean field $S$-type Bose liquid phase probably cannot exist as a stable quantum phase. However, with nonvanishing $d$ eccentricity, both the gauge theory analysis in Sec. VI and the properties of the corresponding $(\operatorname{det})_{x}(\operatorname{det})_{y}$ wave function which we will explore in Sec. IV suggest that the DBL is a stable critical quantum phase.

It is also instructive to examine several other correlation functions within the present mean field treatment. Specifically, consider the boson density-density correlation function,

$$
\begin{aligned}
D_{b}(\mathbf{r}) & =\langle: \delta \hat{\rho}(0) \delta \hat{\rho}(\mathbf{r}):\rangle \\
& =N(N-1)\left\langle\delta^{d}\left(\mathbf{r}_{1}\right) \delta^{d}\left(\mathbf{r}_{2}-\mathbf{r}\right)\right\rangle-\bar{\rho}^{2},
\end{aligned}
$$

where $N$ is the total number of particles. As defined, $D_{b}(\mathbf{r})$ approaches zero for large separations, while negative values at small distances signify a correlation hole. The density structure factor can be calculated as

$$
D_{b}(\mathbf{k})=\int d \mathbf{r} D_{b}(\mathbf{r}) e^{-i \mathbf{k} \cdot \mathbf{r}}=\frac{\left\langle\left|\delta \hat{\rho}_{\mathbf{k}}\right|^{2}\right\rangle}{V}-\bar{\rho},
$$

where $\delta \hat{\rho}_{\mathbf{k}}=\Sigma_{j} \exp \left[-i \mathbf{k} \cdot \mathbf{r}_{j}\right]-N \delta_{\mathbf{k}, \mathbf{0}}$, and $V$ is the system volume.

Microscopically, since $b^{\dagger}=d_{1}^{\dagger} d_{2}^{\dagger}$, for each boson added to the system, both a $d_{1}$ and a $d_{2}$ fermion are added. As such, the boson and fermion densities are equal: $\hat{\rho}_{b}=\hat{\rho}_{d_{1}}=\hat{\rho}_{d_{2}}$. However, in the mean field treatment, the two fermion flavors propagate independently with different Fermi surfaces, so the corresponding fermion density-density correlation functions, which we denote as $D_{d_{\alpha}}^{M F}$, do not coincide. This ambiguity in the density correlation is an intrinsic deficiency 
of the mean field theory. As a crude measure, we approximate the boson density correlation function as an average of that of the $d_{1}$ and $d_{2}$ fermions,

$$
D_{b}^{M F}(\mathbf{r}) \approx \frac{1}{2}\left[D_{d_{1}}^{M F}(\mathbf{r})+D_{d_{2}}^{M F}(\mathbf{r})\right] .
$$

The individual density correlation functions in the mean field theory are simply

$$
D_{d_{\alpha}}^{M F}(\mathbf{r})=-\left|G_{d_{\alpha}}^{M F}(\mathbf{r})\right|^{2} \sim-\frac{1+\cos \left[2 \mathbf{k}_{F_{\alpha}} \cdot \mathbf{r}-3 \pi / 2\right]}{c_{\alpha}|\mathbf{r}|^{3}},
$$

where again $\mathbf{k}_{F_{\alpha}}$ is the place on the $d_{\alpha}$ Fermi surface where the normal is parallel to the observation direction $\hat{\mathbf{r}}$. We recognize the $2 k_{F}$ oscillation with power-law envelope, which in the momentum space translates to a singularity in the structure factor $\sim\left|2 k_{F}-k\right|^{3 / 2}$ across the $2 k_{F}$ line (such $2 k_{F}$ surfaces are illustrated in Fig. 3), while there is also a $|k|$ singularity at zero momentum. As obtained from Eq. (34), the mean field boson density correlator $D_{b}^{M F}$ has singularities at both $2 k_{F_{1}}$ and $2 k_{F_{2}}$, and "knows" about the presence of both Fermi surfaces. Remarkably, this mean field approximation is consistent with our analysis of the $(\operatorname{det})_{x}(\operatorname{det})_{y}$ boson wave function in Sec. IV, which also reveals singular behavior at both $2 k_{F_{1}}$ and $2 k_{F_{2}}$.

Let us now consider the two-boson correlator,

$$
G_{2 b}\left(\mathbf{r}_{1}, \mathbf{r}_{2} ; \mathbf{r}_{1}^{\prime}, \mathbf{r}_{2}^{\prime}\right) \equiv\left\langle b^{\dagger}\left(\mathbf{r}_{1}\right) b^{\dagger}\left(\mathbf{r}_{2}\right) b\left(\mathbf{r}_{1}^{\prime}\right) b\left(\mathbf{r}_{2}^{\prime}\right)\right\rangle,
$$

which injects a pair of bosons at $\mathbf{r}_{1}, \mathbf{r}_{2}$ and removes a pair at $\mathbf{r}_{1}^{\prime}, \mathbf{r}_{2}^{\prime}$. Consider first the limit where the separation between the two injected bosons, $\mathbf{r}=\mathbf{r}_{1}-\mathbf{r}_{2}$, and the two removed bosons, $\mathbf{r}^{\prime}=\mathbf{r}_{1}^{\prime}-\mathbf{r}_{2}^{\prime}$, are both small compared to the mean interparticle spacing, $|\mathbf{r}|,\left|\mathbf{r}^{\prime}\right| \ll k_{F}^{-1}$. Moreover, take the separation $\mathbf{R}=\frac{\mathbf{r}_{1}+\mathbf{r}_{2}}{2}-\frac{\mathbf{r}_{1}^{\prime}+\mathbf{r}_{2}^{\prime}}{2}$ between the injected and removed pair to be much larger than the interparticle spacing, $|\mathbf{R}| \gg k_{F}^{-1}$. In this limit, the mean field two-boson correlator can be expressed as

$$
G_{2 b}^{M F} \sim \frac{k_{F}^{8}}{\left[1+\frac{W}{2} \sin ^{2}(2 \phi)\right]^{5 / 2}} \frac{\Psi_{x y}(\mathbf{r} ; \hat{\mathbf{R}}) \Psi_{x y}^{*}\left(\mathbf{r}^{\prime} ; \hat{\mathbf{R}}\right)}{\left(k_{F} R\right)^{6}},
$$

with "pair wave function,"

$$
\Psi_{x y}(\mathbf{r} ; \hat{\mathbf{R}})=(\mathbf{r} \cdot \hat{\mathbf{R}})^{2}+W \sin (2 \phi) x y .
$$

Here, for concreteness, we specialized to the elliptical Fermi surfaces [Eq. (23)], and $W \geqslant 0$ characterizes the $d$ eccentricity,

$$
W=\frac{w^{4}+(1 / w)^{4}}{2}-1
$$

$\hat{\mathbf{R}}=\mathbf{R} / R=\cos (\phi) \hat{\mathbf{x}}+\sin (\phi) \hat{\mathbf{y}}$ is the unit vector pointing from one pair to the other. With vanishing $d$ eccentricity, $W=0$, the pair wave function is of an "extended-s" form, for example,
$\Psi_{x y} \sim x^{2}$ for $\phi=0$. This corresponds to a quadratic nodal line in the pair wave function. With $W=0$, the directions of the nodal lines for each pair are aligned perpendicular to the vector connecting one pair to the other. On the other hand, in the limit of very large $d$ eccentricity, $W \gg 1$, the pair wave function takes the $d_{x y}$ form, $\Psi_{x y} \sim x y$, corresponding to two nodal lines along the $x$ and $y$ axes. Upon rotating the internal coordinate, the sign of the pair wave function takes the usual $d_{x y}$ form, precisely as for the Cooper pair wave function in a $d_{x y}$ superconductor. Thus, the DBL has quasi-long-range order in the $d_{x y}$ boson pair channel, although the power-law decay exponent within mean field theory is very large.

It is also interesting to examine the time decay of a "local" two-boson Green's function,

$$
G_{2 b}\left(\mathbf{r}, \mathbf{r}^{\prime} ; \tau-\tau^{\prime}\right) \equiv\left\langle b^{\dagger}(\mathbf{r}, \tau) b^{\dagger}(\mathbf{0}, \tau) b\left(\mathbf{r}^{\prime}, \tau^{\prime}\right) b\left(\mathbf{0}, \tau^{\prime}\right)\right\rangle,
$$

with $|\mathbf{r}|,\left|\mathbf{r}^{\prime}\right| \ll k_{F}^{-1}$. A pair of bosons is injected at positions $\mathbf{r}$ and $\mathbf{0}$, and is removed at later times at positions $\mathbf{r}^{\prime}$ and $\mathbf{0}$. Within mean field theory,

$$
G_{2 b}^{M F}\left(\mathbf{r}, \mathbf{r}^{\prime} ; \tau\right) \sim \frac{1}{\tau^{4}}\left[\left(\mathbf{r} \cdot \mathbf{r}^{\prime}\right)^{2}+2 W(x y)\left(x^{\prime} y^{\prime}\right)\right] .
$$

For large $d$ eccentricity, $W \gg 1$, this factorizes into a product of two pair wave functions of the $d_{x y}$ form. This correlator can be used to extract a local pair tunneling density of states,

$$
\rho_{x y}(E)=\int\left\langle\hat{\mathcal{D}}_{x y}^{\dagger}(\tau) \hat{\mathcal{D}}_{x y}(0)\right\rangle e^{-E|\tau|} d \tau,
$$

where $\hat{\mathcal{D}}_{x y}^{\dagger}$ injects a $d_{x y}$ pair centered at the origin,

$$
\hat{\mathcal{D}}_{x y}^{\dagger} \equiv \int_{\mathbf{r}} x y \exp \left(-\frac{|\mathbf{r}|}{\xi}\right) b^{\dagger}(\mathbf{r}) b^{\dagger}(\mathbf{0}),
$$

with pair "size" $\xi$. Within mean field theory, one obtains a power-law tunneling density of states $\rho_{x y}^{M F}(E) \sim W E^{3}$, with an amplitude that grows with the $d$ eccentricity parameter $W$. The tunneling density of states for an $s$-wave pair also vanishes as $E^{3}$, but the amplitude is independent of $W$. This pair tunneling density of states is perhaps the best diagnostic for measuring the degree of local $d$-wave two-boson correlations in the DBL.

Finally, we consider a box correlator for bosons which is defined in Sec. I [Eq. (6)]. Within mean field, this factorizes as $\mathcal{B}_{b}^{M F}(x)=\mathcal{B}_{d_{1}}(x) \mathcal{B}_{d_{2}}(x)$, and from Wick's theorem and $90^{\circ}$ rotational invariance,

$$
\mathcal{B}_{d_{1}}(x)=G_{d_{1}}^{2}(0, x)-G_{d_{1}}^{2}(x, 0)=-\mathcal{B}_{d_{2}}(x) .
$$

Thus,

$$
\mathcal{B}_{b}^{M F}(x)=-\left[G_{d_{1}}^{2}(0, x)-G_{d_{1}}^{2}(x, 0)\right]^{2} \leqslant 0 .
$$

Notice that in the case with zero eccentricity the mean field box correlator vanishes, but since the (det) ${ }^{2}$ wave function is non-negative, one expects $\mathcal{B}_{b}$ to be positive upon inclusion of gauge fluctuations. With nonzero $d$ eccentricity, the mean field box correlator is negative for all spatial separations, which reflects the underlying $d_{x y}$ nodal structure, and decays 
as $x^{-6}$. It is possible that the inclusion of gauge fluctuations will again modify the mean field result in the case with closed Fermi surfaces because of the pairing tendencies of the $d_{1}$ and $d_{2}$ fermions. In the case with open Fermi surfaces, to be discussed next, we conjecture that the exact box correlator will be negative at large spatial separations while decaying to zero as the box size is taken to infinity. This conjecture appears to be consistent with the box correlator extracted from the $(\operatorname{det})_{x}(\operatorname{det})_{y}$ wave function with open Fermi surfaces in Sec. IV B.

\section{Case with open Fermi surfaces}

The preceding analysis holds for arbitrary Fermi surfaces, in particular, for the lattice bands obtained from Eq. (18). We only need to remember that the fermion Green's function Eq. (27) is determined by the Fermi surface patches with the group velocities that are parallel or antiparallel to $\hat{\mathbf{r}}$. Once $G_{d_{1}}$ and $G_{d_{2}}$ are known, the other correlation functions wil follow.

An interesting situation occurs on the lattice when the ratio $t_{\|} / t_{\perp}$ is such that the $d_{1}$ and $d_{2}$ Fermi surfaces are open, which is illustrated in the right panel of Fig. 1. In this case, for an observation direction close to, say, the $y$ axis, there are no $d_{1}$ Fermi surface patches with normals in this direction, so the $d_{1}$ Green's function has an exponential decay in this direction instead of the power law [Eq. (27)]. Since the realspace boson Green's function is the product of the two fermion Green's functions [Eq. (26)], we conclude that it decays exponentially in the directions near the $x$ and $y$ axes. There may still be directions near $\hat{\mathbf{x}} \pm \hat{\mathbf{y}}$ in which both $G_{d_{1}}$ and $G_{d_{2}}$ show power-law behavior, and so will the boson Green's function.

Eventually, for large enough $t_{\|} / t_{\perp}$, the two Fermi surfaces will have no parallel patches, and the boson Green's function will decay exponentially in all directions. We will call this phase DLBL for $d$-wave correlated local Boson liquid. Note, however, that the system is still gapless and critical, as can be measured, e.g., from the local spectral function Eq. (31). Also, the boson density correlations still have the power-law envelope [Eq. (35)] if one fermion field can propagate in the observation direction. Furthermore, the boson box correlator [Eq. (45)], exhibits power-law behavior even though the single and pair boson Green's functions are exponentially decaying. From the boson field perspective, the system is local and bosons have a hard time to propagate; nevertheless, the system has power-law correlations in other properties and, in particular, charges can propagate.

Based on an analysis of gauge fluctuations in Sec. V, we conjecture that the $d_{1}$ and $d_{2}$ systems effectively decouple at low energies in the DLBL, and at large distances, the exact box correlator is negative and decays to zero as a nonoscillatory power law with an integer exponent which is independent of nonuniversal lattice scale physics, $\mathcal{B}_{b}(x) \sim-x^{-8}$. This is the same as in the mean field theory except with a larger power.

It is also worth mentioning the limiting case $t_{\perp}=0$ that gives completely flat Fermi surfaces, which we will call "ex- tremal DLBL." The $d_{1}$ fermions can move only along the $x$ axis,

$$
G_{d_{1}}(x, y)=\delta_{y, 0} \frac{\sin \left(k_{F} x\right)}{\pi x} \quad(\text { extremal DLBL })
$$

while the $d_{2}$ fermions can move only along the $y$ axis. As a result, the boson field cannot propagate at all, not even one lattice spacing. However, this special system still has powerlaw density-density correlations, e.g.,

$$
D_{d_{1}}^{M F}(x, y) \sim-\delta_{y, 0} \frac{\sin ^{2}\left(k_{F} x\right)}{x^{2}} \quad(\text { extremal DLBL }),
$$

as well as power-law box correlation,

$$
\mathcal{B}_{b}^{M F}(x) \sim-\frac{\sin ^{4}\left(k_{F} x\right)}{x^{4}} \quad(\text { extremal DLBL }) .
$$

From the numerical study of the extremal DLBL wave function (Sec. IV B 2), we conjecture that these mean field power laws also hold upon the Gutzwiller projection. In the gauge theory context, we would say that the fermions remain unaffected by the gauge field fluctuations. This extremal DLBL wave function is of interest because of its similarity to the so-called excitonic Bose liquid ground state of a pure ring exchange model-we will discuss this in Sec. VII.

\section{PROPERTIES OF THE WAVE FUNCTIONS}

In this section, we study the $\Psi=(\operatorname{det})_{x}(\operatorname{det})_{y}$ wave functions directly and numerically measure their properties such as the boson Green's function and the density correlation defined in Sec. III B. A detailed comparison is made with the mean field, which provides an initial guide. This is followed by interpretations of the observed deviations from the mean field using simple "Amperean interaction" rules of thumb; the actual calculations behind these rules, within the gauge fluctuations theory, are given in Sec. V.

We first consider wave functions in the continuum so as to avoid lattice effects and focus on the consequences of the underlying Fermi surfaces. It appears that the $S$-type wave function, $\Psi=(\mathrm{det})^{2}$, has off-diagonal long-range order, while a generic DBL wave function with nonzero $d$ eccentricity has only power-law boson correlations. We then consider wave functions on the lattice where we can access the DLBL phase with open Fermi surfaces described in Sec. III C; this features boson Green's function that decays exponentially in real space. Finally, we discuss in some detail the extremal wave function that is obtained when the Fermi surfaces are completely flat.

The calculations with the wave functions are performed numerically using standard determinantal variational Monte Carlo techniques. ${ }^{29}$ The system used in all calculations is a square box with periodic boundary conditions. In each case, the particle number is chosen so as to fill complete momentum shells under the Fermi surfaces. To facilitate the comparison, the presented mean field is calculated for the same finite systems. 


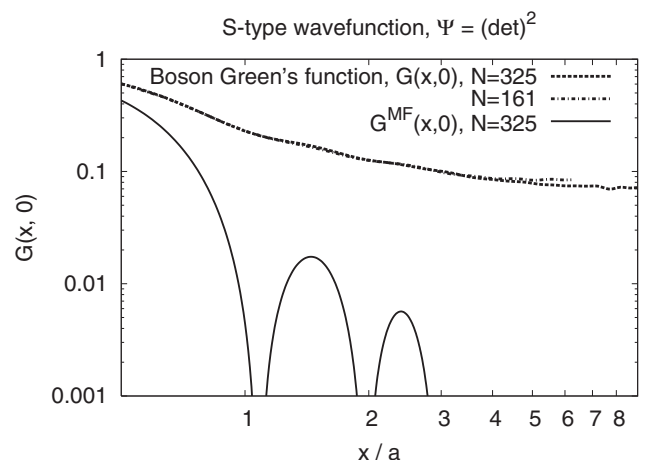

FIG. 4. Boson correlation in the (det) $)^{2}$ wave function for two systems with $N=161$ and 325 particles. The distance is measured in units of $a=\rho^{-1 / 2}$, and the plots are cut when the separation exceeds half of the box size. Since $G_{b}(r)$ maintains a sizable limiting value that depends only weakly on $N$, we suggest that there is offdiagonal long-range order in this wave function. The mean field result is also plotted, and the vertical scale is chosen to accommodate its fast decay, $G_{b}^{M F} \sim 1 / r^{3}$.

\section{A. Wave functions in the continuum}

Before proceeding with the numerics, the DBL structure factors can be found analytically in some ranges in the momentum space: Thus, by expanding the boson wave function in terms of the orbitals that form each determinant, it is easy to see that $n_{\mathbf{k}}$ vanishes outside the $\mathbf{k}_{F 1}+\mathbf{k}_{F 2}$ surface of Fig. 3, while $D(\mathbf{k})$ vanishes outside the $2 \mathbf{k}_{F 1}+2 \mathbf{k}_{F 2}$ surface.

\section{S-type state}

We begin by considering the limit with zero $d$ eccentricity, in which case the boson wave function is positive everywhere except for the nodes. Figure 4 shows boson Green's function measured for two systems with $N=161$ and 325 particles. It appears that at large separations, $G_{b}(r)$ approaches a finite positive value, which is roughly the same for both system sizes. To be more quantitative, the $\mathbf{k}=0$ mode contains $n_{\mathbf{k}=0} / N=0.10$ and 0.083 fractions of bosons in the two systems. The present data extracted from the continuum wave function cannot rule out the possibility that the Green's function vanishes in the large-distance limit. However, at the very least, the result of the projection is clearly dramatic in this case with matched Fermi surfaces, since the falloff of the boson correlation is very slow (if any). We also remark that we unambiguously find off-diagonal long-range order when this wave function is studied on a lattice at fixed boson density.

The extremely strong enhancement of the boson correlation over the mean field prediction seen in Fig. 4 can be understood qualitatively as a result of the pairing of the $d_{1}$ and $d_{2}$ fermions mediated by the gauge field. Indeed, as we will discuss in Sec. V, the constituents of such a (zero momentum) "Cooper pair" with oppositely directed group velocities and opposite gauge charges produce parallel gauge currents and therefore experience Amperean attraction mediated by the gauge field. This "Amperean attraction" rule of thumb for the enhancements in correlations appears to be taken to the extreme in the (det) $)^{2}$ wave function, where we
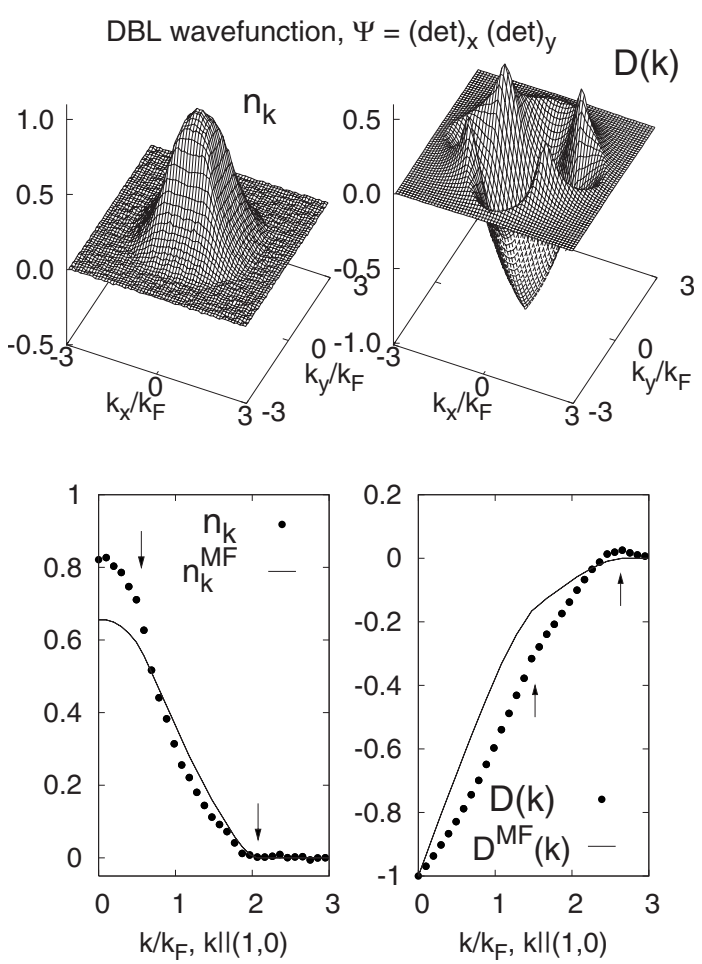

FIG. 5. (Top) Mode occupation $n_{\mathbf{k}}$ and density structure factor $D(\mathbf{k})$ measured in the continuum DBL wave function with $w^{4}=3$ [note that our $D(\mathbf{k})$, defined in Eq. (33), vanishes for large $k$ ]. The boson number is $N=325$; the underlying Fermi surfaces and all singular lines are shown to scale in Fig. 3. (Bottom) Cut through the data in the $(1,0)$ direction in the $k$ space and comparison with the mean field results. In the left panel, the arrows point to the appropriate $k_{F 1} \pm k_{F 2}$ momenta (see Fig. 3), while in the right panel the arrows point to the $2 k_{F 1}$ and $2 k_{F 2}$ momenta.

can crudely picture the fermions paired back into $b$ and condensed, giving rise to long-range order in $G_{b}$. We also point out that this wave function has rather unusual density-density correlations, which are singular around the $2 k_{F}$ circle. Since the presence of the off-diagonal order makes this state less interesting to us, we do not consider such details any further. The observation of the order suggests that the $S$-type state is unstable; the ultimate phase in this case is likely a conventional superfluid, and a good superfluid wave function needs to be constructed differently, e.g., using Jastrow approach that builds in proper density correlations.

\section{DBL state}

We now consider in detail a representative case with nonzero $D$ eccentricity-specifically, with $w^{4}=3$ in Eq. (23). The corresponding $d_{1}$ and $d_{2}$ Fermi surfaces are shown to scale in Fig. 3 together with the singular lines for the order parameter and density correlations identified in the mean field (Sec. III).

Figure 5 gives an overall view of $n_{\mathbf{k}}$ and $D(\mathbf{k})$ in the two-dimensional momentum space, and also shows a onedimensional cut in the $(1,0) k$-space direction together with the mean field predictions. 

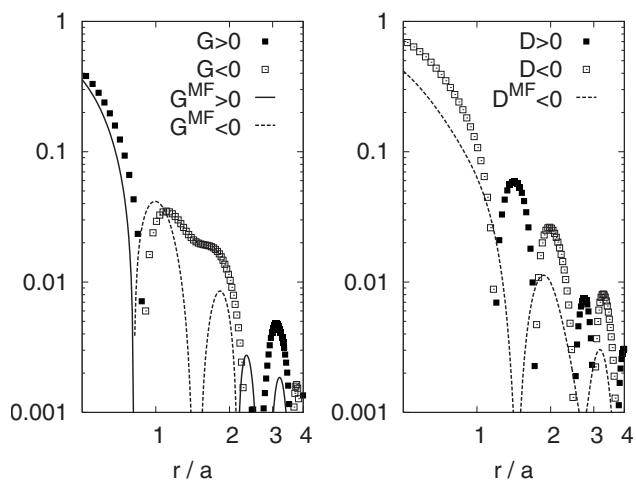

FIG. 6. Boson Green's function $G_{b}(r)$ and density correlation $D_{b}(r)$ measured along the $r=(x, 0)$ direction for the DBL wave function with $N=325$ bosons and $d$ eccentricity characterized by $w^{4}=3$ (this is the same system as in Fig. 5). The distance is measured in units of $a=\rho^{-1 / 2}$, and the plots are cut roughly where the signal in $G_{b}(r)$ approaches the noise level. Note the logarithmic scales used, so it is the absolute values that are plotted, while the positive (negative) sign of the data is indicated with filled (open) symbol. In the case of the mean field data, the signs are indicated using solid (broken) lines; $D^{M F}$ is always negative [see Eq. (35)].

Consider first the mode occupation function $n_{\mathbf{k}}$. Upon projection, this develops a squarish top with somewhat sharper edges as compared with the mean field. The latter is not shown in the full $k$ space but looks smoother, while a $(1,0)$ cut can be seen in the bottom panel of Fig. 5. Coming from large momenta, significant deviations from the mean field set in near the $\mathbf{k}_{F 1}-\mathbf{k}_{F 2}$ line of Fig. 3 [the corresponding location in the one-dimensional (1D) cut is indicated with an arrow]. This surface is singular in the mean field, but the singularities are weak and are almost not visible, while they become more pronounced upon the projection. On the other hand, we observe no such enhancement near the $\mathbf{k}_{F 1}+\mathbf{k}_{F 2}$ line.

This difference between $\mathbf{k}_{F 1}-\mathbf{k}_{F 2}$ and $\mathbf{k}_{F 1}+\mathbf{k}_{F 2}$ is also visible when examining the boson Green's function in real space, as shown in the left panels of Figs. 6 and 7. The mean field $G_{b}^{M F}(\mathbf{r})$ [Eq. (28)] has both $\mathbf{k}_{F 1}-\mathbf{k}_{F 2}$ and $\mathbf{k}_{F 1}+\mathbf{k}_{F 2}$ components equally present, while after the Gutzwiller projection, one finds that the $\mathbf{k}_{F 1}-\mathbf{k}_{F 2}$ component dominates. Indeed, for the boson Green's function measured along the $x$
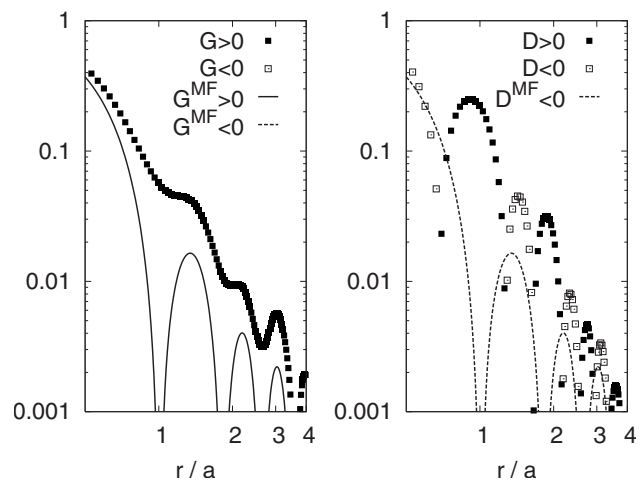

FIG. 7. The same as in Fig. 6 but in the $x=y$ diagonal direction in real space. axis (Fig. 6), the relevant Fermi surface patches have normals in the $(x, 0)$ direction and are easily located (the relevant momentum space cut of $n_{\mathbf{k}}$ is also shown in the left panel of Fig. 5). By comparing with the mean field Eq. (28), one finds that after the projection the amplitude of the oscillation with the smaller wave vector $\left|k_{F 1}-k_{F 2}\right|$ wins over that with the larger wave vector $k_{F 1}+k_{F 2}$. Slightly more care is needed when interpreting $G_{b}(\mathbf{r})$ in the $x=y$ diagonal direction (Fig. 7). In this case, the appropriate locations on the $\mathbf{k}_{F 1}-\mathbf{k}_{F 2}$ surface are at the "cusp" points, with $k_{x}=-k_{y}$ in Fig. 3 , since this is where the normal to the singular surface is parallel to the observation direction $\hat{\mathbf{r}}$. This component does not oscillate when moving along the $x=y$ diagonal in real space, and when it is enhanced, the oscillations due to the other $\mathbf{k}_{F 1}+\mathbf{k}_{F 2}$ component become less visible, which is what we see in Fig. 7.

Unfortunately, from these data we cannot attest whether the enhanced singularities are characterized by different exponents or whether we see only an amplitude effect. The locations where the enhancements occur are consistent with the Amperean rules of thumb (Sec. V). Indeed, the $d_{1}$ and $d_{2}$ constituents of the boson operator $b^{\dagger}=d_{1}^{\dagger} d_{2}^{\dagger}$ move in the opposite directions when contributing to the correlation at $\mathbf{k}_{F 1}$ $-\mathbf{k}_{F 2}$ and therefore experience Amperean attraction and enhancement (remembering that $d_{1}$ and $d_{2}$ carry opposite gauge charges), while they move in the same direction when contributing at $\mathbf{k}_{F 1}+\mathbf{k}_{F 2}$ and therefore experience Amperean suppression. These pictures are made more precise in Sec. V, where we find that within the gauge fluctuations theory, the enhanced correlations are characterized by other exponents that depend on the observation direction because of the varying degree of the Fermi surface curvature matching in the $d_{1}-d_{2}$ pairing channel. The strongest such enhancement is expected along the diagonals $x= \pm y$, which is roughly what we find in the projected wave functions (we repeat again that we cannot make any statement about the exponents from the data other than that we see increased numerical correlations compared with the mean field).

Consider now the density correlation $D_{b}(r)$ and the corresponding structure factor $D_{b}(\mathbf{k})$. In Fig. 5, we can see the singular $2 \mathbf{k}_{F 1}$ and $2 \mathbf{k}_{F 2}$ lines (cf. Fig. 3); the enhancements are peaked where the two curves cross. The singular $2 k_{F}$ points are also visible in the $(1,0) k$-space cut (bottom right panel of Fig. 5). On the other hand, the $|k|$ singularity near zero momentum is not enhanced. Examination of the density correlation in real space (right panels of Figs. 6 and 7) shows an overall increase and dominance of the oscillatory components over the zero-momentum component as compared with the mean field Eq. (35). Again, we cannot tell whether there is a change in the exponents or just an amplitude effect. The $2 k_{F}$ enhancements of the density correlation agree qualitatively with the gauge theory expectations. Indeed, consider the density operator $d_{1}^{\dagger} d_{1}$. The particle and hole constituents (which are oppositely gauge charged) have antiparallel group velocities when contributing to the $2 k_{F}$ component and therefore experience Amperean attraction, while they move in the same direction when contributing at zero momentum and therefore repel each other. Again, these rules of thumb are made more precise in Sec. V, where such enhancements in 
the particle-hole channel are characterized by other direction-dependent exponents (the density correlations are found to decay more slowly when less curved patches are involved). Note that the projection imposes $\hat{\rho}_{b}=\hat{\rho}_{d_{1}}=\hat{\rho}_{d_{2}}$, and we expect $D_{b}$ to acquire both the $2 k_{F 1}$ and $2 k_{F 2}$ signatures (in the gauge theory, $\rho_{d_{1}}$ and $\rho_{d_{2}}$ imprint on each other via nonsingular high-energy connections that are not manifest in our low-energy effective description of Sec. V).

To summarize, the DBL wave function clearly knows about the underlying Fermi surfaces; it also contains germs of the gauge fluctuations theory, since the enhancements or suppressions of the various " $2 k_{F}$ " lines appear to agree with the Amperean rules. However, there are no reasons to believe that the wave function and the gauge theory will have the same long-distance properties. In particular, our measurements cannot tell whether the long-distance power laws are changed upon the projection compared with the mean field. Still, it is gratifying to see that the Amperean rules work for the DBL wave function.

\section{B. Wave functions on the lattice}

We now describe our results for various states on the lattice. As we have already mentioned, $S$-type wave function, $\Psi=(\mathrm{det})^{2}$, with finite boson density per site, has off-diagonal long-range order, which we confirm unambiguously using finite-size scaling. On the other hand, wave functions with nonzero $d$ eccentricity do not show such order. While we have not performed detailed studies, we expect that the case with closed Fermi surfaces is similar to the already described continuum DBL wave function, with a slight complication that one needs to do proper Brillouin zone folding when considering singular surfaces in the momentum space.

\section{Open Fermi surfaces: DLBL state}

Of particular interest is the case with open Fermi surfaces, which can only be realized on the lattice. Specifically, we studied a $24 \times 24$ system with $N=216$ bosons $(\rho=0.375$ per site) and the fermion hopping parameters $t_{\|}=1.35$ and $t_{\perp}$ $=0.65$. In this case, the $d_{1}$ and $d_{2}$ Fermi surfaces have no parallel patches; we therefore expect that the boson Green's function decays exponentially. When we measure $G_{b}(r)$, we find that it drops below our noise level already at three lattice spacings, so the corresponding plots are not particularly informative and are not shown here. Where we can measure reliably, the values after the projection are of the same order as the mean field values in the same system. Since the latter decay exponentially at large distances, we conjecture the same behavior in the DLBL wave function.

On the other hand, the boson density correlation along the $x$ and $y$ axes shows oscillations with a clear power-law envelope, while the correlations are much smaller in the diagonal $x= \pm y$ directions. This behavior is again qualitatively consistent with the mean field, since neither $d_{1}$ nor $d_{2}$ Fermi surfaces have normals in the diagonal directions, while one or the other has normals along the $x$ or $y$ axis.

\section{Flat Fermi surfaces: Extremal DLBL state}

Finally, let us discuss the extremal case when the Fermi surfaces are completely flat. The $d_{1}$ fermions can move only

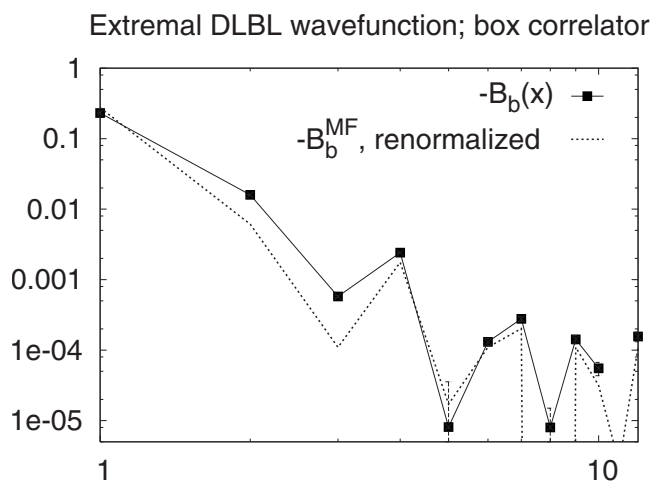

FIG. 8. Box correlator $\mathcal{B}_{b}(x)$ in the extremal DLBL wave function with flat Fermi surfaces on a $24 \times 24$ lattice with $N=216$ bosons (the lines are guides for the eyes). Note that the box correlator is negative in the mean field and is found to be negative in the DLBL state; we plot $-\mathcal{B}_{b}(x)$ to be able to use the logarithmic scale. The mean field Eq. (45) is renormalized as explained in the text using a numerical factor that removes double weighting of configurations. The measured values below $10^{-5}$ are at the noise threshold.

along the $x$ axis and $d_{2}$ fermions only along the $y$ axis. Using fermion orbitals that are localized on individual rows for $d_{1}$ (or columns for $d_{2}$ ), one can see that the boson wave function is nonzero only when the number of particles on each row (or on each column) is the same. Thus, the bosons cannot propagate and their intersite correlation is identically zero. As we will discuss in Sec. VII, pure ring Hamiltonian conserves boson number on each row and/or column, and the extremal DLBL wave function may be useful in this context.

We can still use the box correlator [Eq. (6)] to characterize the state and see some "gaplessness" in the system. The measurement is shown in Fig. 8, where we also plot the renormalized mean field result. The latter is obtained by dividing Eq. (45) by $\rho^{2}(1-\rho)^{2}$, and a crude justification for such procedure ${ }^{30}$ is as follows: Each $d_{1}$ or $d_{2}$ mean field box calculation contains implicitly a weight of order $\rho^{2}(1-\rho)^{2}$, since for the ring operator to be nonzero, two specific sites need to be occupied and two need to be empty. However, after the projection, it is enough to require that only the $d_{1}$ configuration is "correct" since the $d_{2}$ fermions are tied to $d_{1}$. From Fig. 8, we see that the box correlator is negative and tracks closely the renormalized mean field values.

We can also characterize the extremal DLBL state by measuring the density correlations and comparing with the mean field prediction [Eq. (47)]. The results are in Fig. 9. The left panel shows $D_{b}(x, 0)$ as a function of the distance along the $x$ axis: it has stronger oscillatory component than the mean field and swings back and forth across the zero line while the mean field only touches it, but the overall magnitudes are comparable and decay as $1 / x^{2}$. We also find that the density correlation in the $x= \pm y$ diagonal directions decays exponentially (not shown); the mean field predicts zero correlation unless strictly along the axes, and we expect that after the projection this corresponds to exponential decay.

In the right panel of Fig. 9, we show the density structure factor $D(\mathbf{k})$. Several features are clearly visible: rising "tow- 


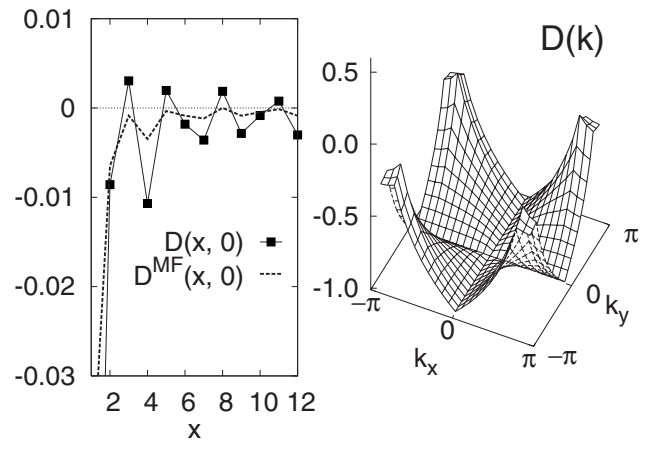

FIG. 9. Boson density correlation for the same extremal DLBL system as in Fig. 8. (Left panel) Real space dependence along the $x$ axis, $D_{b}(x, 0)$, together with the mean field expectation. (Right panel) Density structure factor in the momentum space.

ers" draw attention to the lines $\left( \pm 2 k_{F}, k_{y}\right)$ and $\left(k_{x}, \pm 2 k_{F}\right)$; one can also see a "cross" formed by the lines $\left(0, k_{y}\right)$ and $\left(k_{x}, 0\right)$ that run along the axes. As far as we can say, the character of the singularities across these lines remains the same as in the mean field; there is an amplitude enhancement of the $2 k_{F}$, but no qualitative difference otherwise. In particular, near the line $\left(0, k_{y}\right)$, we observe $D_{b, \text { sing }}\left(k_{x} \rightarrow 0, k_{y}\right)=A\left|k_{x}\right|$ with $A$ independent of $k_{y}$ as long as $\left|k_{y}\right| \gg\left|k_{x}\right|$.

We thus conjecture that the extremal DLBL wave function is adequately described by the free fermion mean field. Some of our findings, e.g., the cross singularity that has a longwavelength character, can be understood semianalytically, since the absolute value of the wave function has a Jastrow form with a peculiar pseudopotential, $u(x, y)$ $\sim-\ln |\sin (\pi x / L)| \delta_{y, 0}-\ln |\sin (\pi y / L)| \delta_{x, 0}$. It seems plausible that one can calculate other properties of this wave function analytically. It is interesting to note that the above suggests a free fermion description of this boson state, perhaps with some constraints that become irrelevant in an infinite system. We also note that the extremal DLBL appears to be a relative of the so-called excitonic bose liquid (EBL) phase predicted in a pure ring model on the square lattice ${ }^{14}$ that we will discuss in Sec. VII; the above therefore suggests that there may be some such description of the EBL in terms of fermions carrying fractional $1 / 2$ charge.

\section{GAUGE FLUCTUATIONS}

We now study the gauge theory using analytic techniques, focusing on the large $K$ limit, where it is reasonable, to ignore the presence of magnetic monopoles in space-time and to expand the cosine term in Eq. (19), treating the gauge field as noncompact. Moreover, in the $K \rightarrow \infty$ limit, the fermions become free and one can put them into a Fermi sea state. A Fermi surface of fermions minimally coupled to a noncompact U(1) gauge field has a considerable history., $51-41$ The $2+1 \mathrm{D}$ such system has been studied most notably as a theory for the spin sector in the uniform resonating valence bond (RVB) phase in the slave boson approach to the high- $T_{c}$ superconductors. It has been argued that such fermion systems have a stable phase that in some crude aspects is similar to a Fermi liquid-for example, it has a finite long-wavelength compressibility and spin susceptibility. However, the system is strikingly different in other aspects and is described by a non-Fermi-liquid fixed point. A scaling description of this fixed point was developed in Refs. 36 and 37.

While we largely follow the earlier work, we find that it is convenient to consider a slight reformulation in which the only uncontrolled approximation is to assume that the gauge field dynamics can be described correctly by the random phase approximation (RPA), retaining only terms quadratic in the gauge field with a singular quadratic kernel. A virtue of this approach is that one thereby obtains a theory which has an $N$-flavor extension that is soluble at $N=\infty$. Moreover, a controlled and systematic perturbation expansion in powers of $1 / N$ can be implemented, which allows one to compute physical properties in terms of nonuniversal "bare" parameters such as the shapes of the Fermi surfaces. We employ this approach to calculate the leading $1 / N$ behavior for both the boson correlator, $G_{b}(\mathbf{r})$, and the density-density correlator, $D_{b}(\mathbf{r})$, in the $d$-wave Bose liquid.

In Sec. VI, we will consider an effective field theory approach, which allows us to check for the stability of the DBL phase that is present at large $N$. Specifically, we study the effects of residual short-range attractive interactions between the two fermion flavors. With vanishing $d$ eccentricity when the Fermi surfaces for both fermion species become the same, the $s$-wave Cooper channel is "nested," and a possible instability toward a paired BCS state seems likely. The resulting state is a bosonic condensate, since $\left\langle b^{\dagger}\right\rangle=\left\langle d_{1}^{\dagger} d_{2}^{\dagger}\right\rangle \neq 0$. However, a nonvanishing $d$ eccentricity of the Fermi surfaces destroys the BCS nesting and the possible pairing instability. Indeed, we will find that the DBL with a large $d$ eccentricity can exist as a stable phase. (For smaller $d$ eccentricity, an instability toward a finite momentum Bose condensate or an incommensurate charge-density wave coexisting with the DBL is a possibility.) As is discussed in Sec. IV, this is nicely in line with the properties of the associated Gutzwiller wave functions: The (det) ${ }^{2}$ wave function that is obtained in the limit of zero $d$ eccentricity appears to have off-diagonal long-range order, whereas the general $(\operatorname{det})_{x}(\operatorname{det})_{y}$ wave function exhibits power-law correlations consistent with the DBL, as extracted from the gauge theory.

\section{A. Formulation}

Being interested in the low-energy properties, it is legitimate to focus on the fermions living near the Fermi surfaces, just as in Fermi-liquid theory. Moreover, the longitudinal Coulomb interactions mediated by the gauge field are readily screened out within a RPA treatment, generating shortranged screened density-density interactions. However, the transverse fluctuations of the gauge field-the photon-are incompletely screened by the fermion particle-hole excitations. It is thus adequate to just retain the transverse components of the vector potential. Working in the Coulomb gauge with $\boldsymbol{\nabla} \cdot \mathbf{a}=0$, the vector potential reduces to a scalar, e.g., $\mathbf{a}(\mathbf{k})=a(\mathbf{k}) \operatorname{sign}\left(k_{y}\right)\left(k_{y} \hat{\mathbf{x}}-k_{x} \hat{\mathbf{y}}\right) /|\mathbf{k}|$.

Moreover, for each fermion species, it will be sufficient to focus on a pair of Fermi surface patches with normals parallel to some axis, say $\hat{\mathbf{x}}$, as shown in Fig. 10. It is the $a_{x}$ 

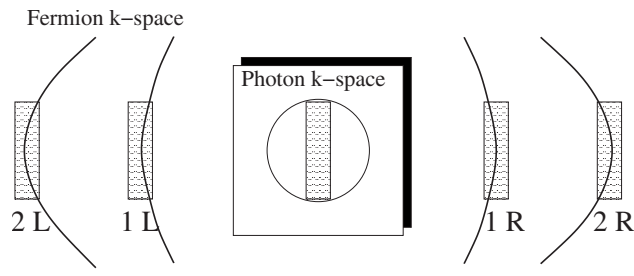

FIG. 10. Relevant momentum patches of the strongly coupled fermion-transverse gauge field system. For simplicity, here and throughout the text, we take the Fermi surface patches with normals in the $\hat{\mathbf{x}}$ direction. The required properties are the Fermi velocities and surface curvatures; the latter are crucial since differently oriented patch systems effectively decouple at low energies.

component of the vector potential which is minimally coupled to these fermions. The important wave vectors of the gauge field $a_{x}(\mathbf{k})$ that are strongly Landau damped by the fermions in these patches satisfy $k_{x} \ll k_{y}$ (see Fig. 10), and in this region of momentum space $a_{x}(\mathbf{k}) \approx a(\mathbf{k})$. Conversely, the modes of the gauge field $a(\mathbf{k})$ with $k_{x} \ll k_{y}$ feed back and scatter the patch fermions. Thus, we can focus on fermion fields $d_{1}(\mathbf{k}), d_{2}(\mathbf{k})$ and the gauge field $a(\mathbf{k})$ that are confined to their respective patches in momentum space, as shown schematically in Fig. 10.

For concreteness, we assume that for each fermion species there are two relevant patches on the opposite sides of the Fermi surface that are labeled $s=R / L= \pm$ for the right/left patch as indicated in Fig. 10. We then decompose the Fermion fields into right and left movers by writing

$$
d_{\alpha}(\mathbf{r}) \sim \sum_{s} e^{i s \mathbf{k}_{F} \cdot \mathbf{r}} d_{\alpha s}(\mathbf{r})
$$

Here, $\mathbf{k}_{F_{\alpha}}$ denote the locations on the two Fermi surfaces which have normals aligned along the $x$ axis, and the fields $d_{\alpha s}(\mathbf{r})$ are assumed to be slowly varying on the scale of $k_{F}^{-1}$.

The full low-energy action consists of three terms describing the dynamics of the gauge field, of the fermions, and of their interactions:

$$
S^{(0)}=\int d^{2} \mathbf{r} d \tau\left[\mathcal{L}_{d}+\mathcal{L}_{a}^{(0)}+\mathcal{L}_{\text {int }}\right]
$$

The "patch" Lagrangian density for the fermions is simply

$$
\mathcal{L}_{d}=\sum_{\alpha} \sum_{s= \pm} d_{\alpha s}^{\dagger}\left[\partial_{\tau}+s v_{\alpha}\left(-i \partial_{x}\right)+\frac{v_{\alpha} c_{\alpha}}{2}\left(-i \partial_{y}\right)^{2}\right] d_{\alpha s},
$$

which is characterized by the local Fermi velocities, $v_{\alpha}$, $(\alpha=1,2)$, and the local Fermi surface curvatures, $c_{\alpha}$. The curvature is crucial in the fermion-gauge problem since there is an effective decoupling of the fermions outside the patch from the gauge field, $a=a_{x}$, which is parallel to the patch normals. Here, $s= \pm 1$ specifies the sign of the group velocity while $v$ will always denote the absolute value.

The dynamics for the transverse gauge field describes the free photon,

$$
\mathcal{L}_{a}^{(0)}=\frac{1}{2}\left[\left(\partial_{\tau} a\right)^{2}+\kappa(\nabla a)^{2}\right],
$$

whereas the gauge-fermion interactions are of the usual minimal coupling form:

$$
\mathcal{L}_{\text {int }}=\sum_{\alpha, s} g_{\alpha} s v_{\alpha} d_{\alpha s}^{\dagger} a d_{\alpha s}
$$

Here, $g_{1}=-g_{2} \equiv g$ with $g=1$, but it will be convenient for bookkeeping purposes to retain $g$ as a dimensionless parameter in the theory. We emphasize that both the fermion fields and the gauge field, which enter the above action in real space, are slowly varying fields corresponding to momentum modes inside their respective patches; in what follows, we always specify the wave vectors of the slow fields relative to their respective patch centers.

\section{B. RPA for the gauge field dynamics}

The above theory is strongly coupled and cannot be treated perturbatively in $g$. As such, it is necessary to resort to approximations to make any meaningful progress. Here, we make our central approximation, namely, replacing the dynamics of the gauge field by a Landau damped form, which results if one sums the RPA bubble of particle-hole excitations: $S_{a}^{(0)} \rightarrow S_{a}$ with

$$
S_{a}=\frac{1}{2} \int_{\mathbf{q} \omega}\left(\Gamma \frac{|\omega|}{\left|q_{y}\right|}+\chi\left|q_{y}\right|^{2}\right)|a(\mathbf{q}, \omega)|^{2} .
$$

The momentum-frequency integral is understood as $\int_{\mathbf{q} \omega}$ $\equiv \int d^{2} \mathbf{q} d \omega /(2 \pi)^{3}$, with the momentum restricted to a region satisfying $\left|q_{x}\right|<\Lambda_{x}$ and $\left|q_{y}\right|<\Lambda_{y}$, with $\Lambda_{x} \ll \Lambda_{y}$.

The magnitude of the Landau damping coefficient $\Gamma$ will be determined by the low-energy fermions near the specified Fermi surface patches. Within the RPA, one obtains

$$
\Gamma_{R P A}=\frac{g^{2}}{2 \pi}\left(\frac{1}{c_{1}}+\frac{1}{c_{2}}\right),
$$

but we will retain $\Gamma$ as an independent parameter since, beyond this point, we will not be using RPA in any event. It will sometimes prove convenient to consider the limit in which the dimensionless gauge charge,

$$
e^{2} \approx \frac{g^{2}}{\Gamma c}
$$

is small. Here, $c$ is a characteristic Fermi surface curvature. Indeed, within the $N$-flavor extension discussed below, while $1 / N$ will be the small parameter that leads to a controlled analysis, already at order $1 / N$ there are some physical properties which we can only compute analytically when $e^{2}$ is simultaneously taken as a small parameter.

The parameter $\chi$ that enters in $S_{a}$ is a "stiffness," which has to be positive for stability. Starting from the lattice gauge theory formulation, $\chi$ gives a measure of the energetic cost of setting up static internal fluxes. One can very crudely estimate $\chi$ using the expression for the diamagnetic response of free fermions, 


$$
\chi \approx \frac{g^{2}}{12 \pi m_{d}},
$$

where $m_{d}$ is some effective mass. We reiterate that this stiffness comes from some energetics that is assumed to stabilize the Fermi surfaces of the $d$ fermions in the first place. Therefore, from the low-energy perspective, $\chi$ should be viewed as a phenomenological parameter that encodes some highenergy physics. Furthermore, the stiffness $\chi$ is independent of the patch orientation in momentum space for the transverse gauge field $a(\mathbf{k})$, since gauge invariance requires that the field energy be proportional to $\chi B^{2}=\chi\left(\partial_{x} a_{y}-\partial_{y} a_{x}\right)^{2}$. In any event, the magnitude of $\chi$ will play a minor role below. Indeed, for correlation functions with power law decay as we find below, changing $\chi$ will only modify the amplitude but not the form of the correlators.

Before proceeding with an analysis of the full theory, $S_{d}$ $+S_{a}+S_{i n t}$, it is important to note that within the above approximation the Landau damped dynamics of the gauge field described by the action $S_{a}$ is both singular and harmonic. We have ignored possible terms in the action which involve higher powers of the gauge field. As such, at this stage, we could integrate out the gauge field exactly, generating a nonlocal and retarded four-fermion interaction. It is the absence of nonlinearities for the gauge field dynamics which allows us to introduce a large- $N$ generalization which is soluble at $N=\infty$ and which facilitates a formal and systematic $1 / N$ expansion, as we now describe.

\section{C. $N$-flavor extension}

Purely for technical reasons, then, we generalize the theory to $N$ flavors of fermions at the two patches on each Fermi surface, $d_{\alpha s} \rightarrow d_{i \alpha s}$. Here, $i=1, \ldots, N$ labels the flavor index, while $s= \pm$ labels the patch location and $\alpha=1,2$ the two Fermion species. The action for the fermions is simply generalized as

$$
S_{d}^{(N)}=\sum_{i=1}^{N} \sum_{\alpha, s} \int_{\mathbf{x} \tau} d_{i \alpha s}^{\dagger}\left(\partial_{\tau}-i s v_{\alpha} \partial_{x}-\frac{v_{\alpha} c_{\alpha}}{2} \partial_{y}^{2}\right) d_{i \alpha s} .
$$

The real scalar gauge field $a(\mathbf{r})$ is likewise generalized, but now as an $N \times N$ Hermitian matrix of complex fields, $a_{i j}(\mathbf{r})$ $=a_{j i}^{*}(\mathbf{r})$. When expressed in momentum space, each component of the matrix, $a_{i j}(\mathbf{q})$, has dynamics with a Landau damped form,

$$
S_{a}^{(N)}=\frac{1}{2} \sum_{i, j=1}^{N} \int_{\mathbf{q} \omega}\left(\Gamma \frac{|\omega|}{\left|q_{y}\right|}+\chi\left|q_{y}\right|^{2}\right)\left|a_{i j}(\mathbf{q}, \omega)\right|^{2} .
$$

Since $a_{i j}(\mathbf{q}, \omega)=a_{j i}^{*}(-\mathbf{q},-\omega)$, this can also be rewritten as a trace over the flavor indices of the matrix $\overleftrightarrow{a}(\mathbf{q}, \omega)$ :

$$
S_{a}^{(N)}=\frac{1}{2} \int_{\mathbf{q} \omega}\left(\Gamma \frac{|\omega|}{\left|q_{y}\right|}+\chi\left|q_{y}\right|^{2}\right) \operatorname{Tr}[\overleftrightarrow{a}(\mathbf{q}, \omega) \overleftrightarrow{a}(-q,-\omega)] .
$$

Finally, the fermion-gauge field interaction is taken as

$$
S_{i n t}^{(N)}=\sum_{\alpha, s} \frac{g_{\alpha}}{\sqrt{N}} s v_{\alpha} \sum_{i, j=1}^{N} \int_{\mathbf{x} \tau} d_{i \alpha s}^{\dagger} a_{i j} d_{j \alpha s} .
$$

Notice that the magnitude of the interaction strength (the charge) has been taken to scale as $1 / \sqrt{N}$. At $N=1$, the theory reduces to that discussed in the previous section, with the assumption of a harmonic Landau damped form for the gauge field dynamics as the only approximation.

It is worth pointing out that the full action has an enlarged global symmetry being invariant under

$$
d_{i \alpha s} \rightarrow e^{i \theta_{i}} e^{i \phi_{\alpha}} d_{i \alpha s}, \quad a_{i j} \rightarrow e^{i\left(\theta_{i}-\theta_{j}\right)} a_{i j},
$$

for arbitrary phases $\theta_{i}, i=1, \ldots, N$, and $\phi_{\alpha}, \alpha=1,2$. Thus, the densities $\Sigma_{\alpha} d_{i \alpha}^{\dagger} d_{i \alpha}$ are independently conserved for any $i$, and also $\Sigma_{i} d_{i \alpha}^{\dagger} d_{i \alpha}$ are independently conserved for any $\alpha$. Note also that the dynamics of each of the $N$-flavor fermions $d_{i 1}$ is identical to one another, and similarly for all the $d_{i 2}$ fermions.

Before solving the full $N$-flavor theory, $S_{d}^{(N)}+S_{a}^{(N)}+S_{i n t}^{(N)}$, exactly at $N=\infty$, we define large- $N$ generalizations of the various correlation functions. As in the gauge mean field theory, we anticipate that the exact fermion Green's function for (any) one of the $N$ fermion flavors, which we denote $G_{d_{\alpha}}^{(N)}(\mathbf{r})$, will be dominated at large distances by the patch fermions with normals parallel or antiparallel to the observation direction $\hat{\mathbf{r}}$. We can thus expand $G_{d_{\alpha}}^{(N)}(\mathbf{r})$ for large $|\mathbf{r}|$ in terms of the patch fermion Green's functions as

$$
G_{d_{\alpha}}^{(N)}(\mathbf{r}, \tau) \approx \sum_{s} e^{i s \mathbf{k}_{F_{\alpha}} \cdot \mathbf{r}} G_{\alpha s}^{(N)}(\mathbf{r}, \tau),
$$

with the definition

$$
G_{\alpha s}^{(N)}\left(\mathbf{r}-\mathbf{r}^{\prime}, \tau-\tau^{\prime}\right)=\left\langle d_{i \alpha s}^{\dagger}(\mathbf{r}, \tau) d_{i \alpha s}\left(\mathbf{r}^{\prime}, \tau^{\prime}\right)\right\rangle .
$$

Note that there is no summation over $i$; throughout, any summation over indices will be shown explicitly.

There are now $N$ flavors of bosons with creation operators

$$
b_{i}^{\dagger}(\mathbf{r})=d_{i 1}^{\dagger}(\mathbf{r}) d_{i 2}^{\dagger}(\mathbf{r}),
$$

with all $N$ boson flavors having identical dynamics. The large- $N$ generalization of the boson correlator is then simply

$$
G_{b ; i j}^{(N)}\left(\mathbf{r}-\mathbf{r}^{\prime}, \tau-\tau^{\prime}\right)=\left\langle b_{i}^{\dagger}(\mathbf{r}, \tau) b_{j}\left(\mathbf{r}^{\prime}, \tau^{\prime}\right)\right\rangle .
$$

Due to the global symmetries, the correlators which are offdiagonal in the flavor indices vanish, leaving a uniquely defined boson Green's function, $G_{b ; i j}^{(N)}=\delta_{i j} G_{b}^{(N)}$. As desired, $G_{b}^{(N)}$ reduces at $N=1$ to the boson correlator defined in Sec. III B.

The boson density-density correlator, $D_{b}^{(N)}$, is readily defined in terms of (any) the boson densities $\hat{\rho}_{i b}$ as in Eq. (32). Similarly, we can define the fermion density-density correlators for each species, $D_{d_{\alpha}}^{(N)}$, in terms of any one of the fermion densities, $\hat{\rho}_{i \alpha}$. As before, we assume that the boson densitydensity correlator can be approximated as an average over $\alpha$ of the fermion density-density correlators, $D_{b}^{(N)} \approx \frac{1}{2} \Sigma_{\alpha} D_{d_{\alpha}}^{(N)}$.

In order to extract the above correlation functions, it is convenient to define patch fermion particle-hole and particleparticle bubbles, which we denote as $\Pi_{p h}^{(N)}$ and $\Pi_{p p}^{(N)}$, respectively. The exact bubble correlators can be formally ex- 
pressed in terms of the exact patch fermion Green's functions and the exact particle-hole and particle-particle vertices,

$$
\begin{aligned}
\Pi_{p h ; \alpha s ; \alpha^{\prime} s^{\prime}}^{(N)}(\mathbf{q}, \omega)= & \int_{\mathbf{q}^{\prime} \omega^{\prime}} V_{p h ; \alpha s ; \alpha^{\prime} s^{\prime}}^{(N)}\left(\mathbf{q}+\mathbf{q}^{\prime}, \omega+\omega^{\prime} ; \mathbf{q}^{\prime}, \omega^{\prime}\right) \\
& \times G_{\alpha s}^{(N)}\left(\mathbf{q}+\mathbf{q}^{\prime}, \omega+\omega^{\prime}\right) G_{\alpha^{\prime} s^{\prime}}^{(N)}\left(\mathbf{q}^{\prime}, \omega^{\prime}\right),
\end{aligned}
$$

$$
\begin{aligned}
\Pi_{p p ; \alpha s ; \alpha^{\prime} s^{\prime}}^{(N)}(\mathbf{q}, \omega)= & \int_{\mathbf{q}^{\prime} \omega^{\prime}} V_{p p ; \alpha s ; \alpha^{\prime} s^{\prime}}^{(N)}\left(\mathbf{q}+\mathbf{q}^{\prime}, \omega+\omega^{\prime} ; \mathbf{q}^{\prime}, \omega^{\prime}\right) \\
& \times G_{\alpha s}^{(N)}\left(\mathbf{q}+\mathbf{q}^{\prime}, \omega+\omega^{\prime}\right) G_{\alpha^{\prime} s^{\prime}}^{(N)}\left(-\mathbf{q}^{\prime},-\omega^{\prime}\right) .
\end{aligned}
$$

Here, $V_{p h}^{(N)}$ and $V_{p p}^{(N)}$ denote the fully renormalized vertices, and $\alpha, s$ and $\alpha^{\prime}, s^{\gamma}$ label the patch locations of the two fermion lines that come out of the vertex. The total momentum and frequency running through the bubble is $\mathbf{q}, \omega$, and is divided between the two fermions as shown above. At $N$ $=\infty$, there are no vertex corrections, $V_{p h}^{(\infty)}=1$ and $V_{p p}^{(\infty)}=1$, and each bubble is just a convolution of the two Green's functions.

The boson correlator at large distances and long times can be readily expressed in terms of the bubbles,

$$
G_{b}^{(N)}\left(s \mathbf{k}_{F_{1}}+s^{\prime} \mathbf{k}_{F_{2}}+\mathbf{q}, \omega\right) \approx \Pi_{p p ; 1 s ; 2 s^{\prime}}^{(N)}(\mathbf{q}, \omega),
$$

and similarly for the fermion density-density correlator,

$$
D_{d_{\alpha}}^{(N)}\left(s \mathbf{k}_{F_{\alpha}}-s^{\prime} \mathbf{k}_{F_{\alpha}}+\mathbf{q}, \omega\right) \approx \Pi_{p h ; \alpha s ; \alpha s^{\prime}}^{(N)}(\mathbf{q}, \omega) .
$$

\section{Solution at $N=\infty$}

At $N=\infty$, the fermion Green's function $G_{\alpha s}^{(N)}(\mathbf{q}, \omega)$ can be readily obtained by summing the nested rainbow diagrams to give

$$
G_{\alpha s}^{(\infty)}(\mathbf{q}, \omega)=\frac{1}{i \omega\left(1+\left|\Omega_{\alpha} / \omega\right|^{1 / 3}\right)-s v_{\alpha} q_{x}-\left(v_{\alpha} c_{\alpha} / 2\right) q_{y}^{2}}
$$

with

$$
\Omega_{\alpha}^{1 / 3}=\frac{v_{\alpha}}{2 \pi \sqrt{3}} \frac{g^{2}}{\chi^{2 / 3} \Gamma^{1 / 3}}
$$

Because of the gauge interactions, each fermion species obtains an anomalous self-energy of the form $-i \omega|\Omega / \omega|^{1 / 3}$, which dominates over the bare term $-i \omega$ on energy scales below $\Omega$. Physically, the fermions cease to propagate as free particles due to the strong scattering from the dynamical gauge field, becoming "incoherent."

We first consider the long-distance and time behaviors of $G_{\alpha s}^{(\infty)}(\mathbf{r}, \tau)$. As in the mean field (Sec. III), the Green's function is dominated at large distances by the patches of the Fermi surface with normals along $\pm \hat{\mathbf{r}}$. For the equal-time Green's function, we obtain

$$
G_{d_{\alpha}}^{(\infty)}(\mathbf{r}, 0) \approx \frac{3 \sqrt{\pi}}{4}\left(\frac{v_{\alpha}}{\Omega_{\alpha}}\right)^{1 / 2} \frac{1}{\sqrt{|\mathbf{r}|}} G_{d_{\alpha}}^{M F}(\mathbf{r}),
$$

where the velocity $v_{\alpha}$ and curvature $c_{\alpha}$ characterize the $d_{\alpha}$ Fermi surface patches with normals along $\pm \hat{\mathbf{r}}$, and $G_{d_{\alpha}}^{M F}(\mathbf{r})$ is given in Eq. (27). On the other hand, the long time dependence of the local fermion Green's function is not affected by the anomalous self-energy and is determined solely by the density of states $\nu_{0}$ at the Fermi energy,

$$
G_{d_{\alpha}}^{(\infty)}(\mathbf{0}, \tau)=-\frac{\nu_{0}}{\tau} .
$$

Turning to the physical correlators, at $N=\infty$, there are no vertex corrections to the fermion bubble, so we simply have

$$
D_{d_{\alpha}}^{(\infty)}(\mathbf{r})=-\left|G_{d_{\alpha}}^{(\infty)}(\mathbf{r})\right|^{2},
$$

which gives for the $N=\infty$ equal-time density correlator

$$
D_{b}^{(\infty)}(\mathbf{r}) \approx \frac{1}{2} \sum_{\alpha} \frac{9 \pi}{16} \frac{v_{\alpha}}{\Omega_{\alpha}} \frac{1}{|\mathbf{r}|} D_{d_{\alpha}}^{M F}(\mathbf{r}),
$$

where $D_{d_{\alpha}}^{M F}(\mathbf{r})$ is given in Eq. (35). Similarly, in the absence of vertex corrections at $N=\infty$ in the particle-particle bubble, one has for the equal-time boson Green's function

$$
\begin{aligned}
& G_{b}^{(\infty)}(\mathbf{r})=G_{d_{1}}^{(\infty)}(\mathbf{r}) G_{d_{2}}^{(\infty)}(\mathbf{r}) \\
\approx & \frac{9 \pi}{16}\left(\frac{v_{1} v_{2}}{\Omega_{1} \Omega_{2}}\right)^{1 / 2} \frac{1}{|\mathbf{r}|} G_{b}^{M F}(\mathbf{r}),
\end{aligned}
$$

where $G_{b}^{M F}(\mathbf{r})$ is given in Eq. (28). However, the time dependence of the local boson Green's function $G_{b}(\mathbf{0}, \tau)$ is unchanged from the mean field Eq. (31). Finally, the box correlator is given by Eq. (45), with appropriate $N=\infty$ fermion Green's functions and decays with a power-law envelope of $-x^{-8}$.

Notice that both the boson and the boson density-density correlators at $N=\infty$ fall off in space as $|\mathbf{r}|^{-4}$, which is faster than their mean field counterparts. Injecting a boson corresponds to creating two fermions. In the mean field, these fermions propagate independently and as free particles. On the other hand, in the present $N=\infty$ theory that sums the nested rainbow diagrams, both fermions propagate "incoherently" due to their scattering from the gauge field, which effectively reduces the ability of the created boson to propagate, thereby leading to a faster decay of the boson correlator. At this level of approximation, the two injected fermions, while scattered by the gauge fluctuations, do not scatter off one another. The effects of interactions between the two injected fermions appear at order $1 / N$, entering as vertex corrections. Since the two fermion species have opposite gauge charge, one expects that they will attract one another. As we shall see, though, they do not form a composite boson (a bound state, for example), but their motion becomes strongly correlated, allowing them to propagate more effectively when spatially close by. The net result, as we find below, is that the boson can propagate more effectively when the motion of the fermion pair is correlated, leading to a slower decay of the correlator compared to the $N=\infty$ result. 
Thus, the effect on the boson dynamics, due to the decoherence experienced by the fermions from scattering off the gauge fluctuations, is compensated, perhaps only partially, by the attractive interaction between the pair of fermions.

\section{E. Fermion Green's function at order $1 / N$}

The $1 / N$ contributions to the fermion Green's function are obtained by considering the nested rainbow diagrams and replacing one gauge field propagator $G_{a}(\mathbf{q}, \omega)=\left(\Gamma|\omega| /\left|q_{y}\right|\right.$ $\left.+\chi\left|q_{y}\right|^{2}\right)^{-1}$ with the bubble correction $\delta G_{a} \sim \frac{1}{N} G_{a}^{2} \Pi_{p h}^{(\infty)}$. Formally, we can first calculate the rainbows using $G_{a}+\delta G_{a}$ everywhere and then extract the $1 / N$ piece. As we now argue, the functional form of the fermion Green's function in Eq. (71) remains unchanged except for finite $1 / N$ shifts in the parameters. Indeed, evaluating the $N=\infty$ particle-hole bubble at small wave vector and frequency gives $\Pi_{p h}^{(\infty)}$ $\times(\mathbf{q}, \omega) \sim|\omega| /\left|q_{y}\right|$, which only leads to a finite $1 / N$ shift in $\Gamma$. This is not surprising, since the bare singular gauge propagator postulated at the outset in our theory is motivated by the free fermion particle-hole bubble, and one can verify that the bubble remains unchanged also in the presence of an arbitrary fermion self-energy that depends on the frequency only. Then, using this renormalized gauge propagator to evaluate the rainbow diagram gives a contribution to the fermion Green's function of the form, $\delta G_{d_{\alpha}}^{(N)} \sim \frac{1}{N} \omega^{2 / 3}\left[G_{d_{\alpha}}^{(\infty)}\right]^{2}$. This will, at most, give finite $1 / N$ shifts to $\Omega_{\alpha}, v_{\alpha}$, and $c_{\alpha}$. Next we consider the vertices at $1 / N$, which undergo more dramatic modifications.

\section{F. Vertices at order $1 / N$}

\section{Single photon contribution}

Consider first dressing the bare vertices with a single gauge propagator. We denote the value of the corresponding Feynman diagrams as $V_{p h}^{(1 a)}$ and $V_{p p}^{(1 a)}$, where $(1 a)$ stands for one (Landau damped) photon exchanged. These diagrams are the first ones in Figs. 11 and 12. As we will discuss below, the full structure at $1 / N$ is more involved, but the calculation of $V^{(1 a)}$ already captures the main qualitative physics.

For simplicity, we first focus on the situation with zero external $y$ momentum and frequency, defining

$$
V_{q_{x}^{-}}^{(1 a)}=\left.V^{(1 a)}\left(\mathbf{q}, \omega ; \mathbf{q}^{\prime}, \omega^{\prime}\right)\right|_{q_{y}=q_{y}^{\prime}=\omega=\omega^{\prime}=0},
$$

where $\mathbf{q}, \mathbf{q}^{\prime}$ and $\omega, \omega^{\prime}$ denote the momenta and frequencies in and out of the two legs, respectively, and $q_{x}^{-}=q_{x}-q_{x}^{\prime}$ is the momentum running through the vertex. For notational simplicity, we have suppressed the corresponding flavor labels $\alpha, s$ and $\alpha^{\prime}, s^{\prime}$. Evaluating the Feynman diagrams gives

$$
\begin{gathered}
V_{p h ; q_{x}^{-}}^{(1 a)}=\frac{1}{N} \operatorname{sign}\left(g_{\alpha} g_{\alpha^{\prime}}\right) \delta_{s,-s^{\prime}} \lambda_{p h} \ln \left(\frac{\Lambda_{x}}{\left|q_{x}^{-}\right|}\right), \\
V_{p p ; q_{x}^{-}}^{(1 a)}=-\frac{1}{N} \operatorname{sign}\left(g_{\alpha} g_{\alpha^{\prime}}\right) \delta_{s,-s^{\prime}} \lambda_{p p} \ln \left(\frac{\Lambda_{x}}{\mid q_{x}^{-}}\right),
\end{gathered}
$$

revealing a logarithmic divergence cutoff by the total $x$ momentum running through the vertex. Explicit expressions for the dimensionless non-negative coefficients $\lambda_{p h}$ and $\lambda_{p p}$ are given below.

Notice that both the particle-hole and the particle-particle vertex corrections vanish unless the two patches have opposite group velocities. Moreover, the sign of the correction is given by the sign of the product of the two charges and differs for the particle-hole and particle-particle channels. The origin of these signs is essentially Ampere's law, familiar from electrodynamics. Two moving charges producing parallel charge currents experience an attractive interaction, bringing them closer together and enhancing their interaction strength, which is encapsulated by the magnitude of the interaction vertex. For example, a particle and a hole of the same fermion species $\left(\alpha=\alpha^{\prime}\right)$ residing on opposite Fermi surface patches $\left(s=-s^{\prime}\right)$ have an enhanced interaction strength, which is encoded in $V_{p h}^{(1 a)}>0$. On the other hand, the interaction vertex is suppressed when the charge currents are antiparallel. For example, a pair of particles with the same charge $\left(g=g^{\prime}\right)$ experience a suppressed vertex interaction, $V_{p p}^{(1 a)}<0$, when their group velocities are antiparallel.

The absence of singular vertex corrections when the two patch fermions have parallel group velocities, $s=s^{\prime}$, is, we believe, not just a peculiarity of this lowest order contribution, but will be valid generally for the exact vertices at arbitrary $N$. This expectation is based on the specific form of the propagators: the fermion propagator has simple poles in the complex $q_{x}$ plane, while the gauge field propagator is independent of $q_{x}$; the latter is a reflection of the transverse nature of the gauge field. For any given Feynman diagram, it is the sign of the internal frequency variables running around the loops together with $s, s^{\prime}$ which will determine whether the poles in the integrand will be in the upper or lower complex $q_{x}$ planes. For vertices with $s=s^{\prime}$, all of the fermion lines internal to any diagram will have the same group velocity, and with energy (frequency) conservation, one expects that at least one of the $q_{x}$ variables will only have poles in the upper (or lower) half plane, so that the contour can be appropriately deformed to show that the diagram vanishes. Thus, we henceforth restrict attention exclusively to vertices with $s=-s^{\prime}$ and, to ease the notation, will drop the explicit $s, s^{\prime}$ indices in the following.

The dimensionless magnitudes of the vertex enhancements in the particle-hole and particle-particle channels, $\lambda_{p h}$ and $\lambda_{p p}$, respectively, are given by

$$
\begin{gathered}
\lambda_{p h}=\frac{g^{2}}{\sqrt{3} \pi \Gamma^{1 / 3} \chi^{2 / 3}\left(\frac{\Omega^{1 / 3}}{v}+\frac{\Omega^{\prime 1 / 3}}{v^{\prime}}\right)} \mathcal{E}\left[\zeta_{p h}\right], \\
\lambda_{p p}=\frac{g^{2}}{\sqrt{3} \pi \Gamma^{1 / 3} \chi^{2 / 3}\left(\frac{\Omega^{1 / 3}}{v}+\frac{\Omega^{\prime 1 / 3}}{v^{\prime}}\right)} \mathcal{E}\left[\zeta_{p p}\right], \\
\lambda_{p h}=\mathcal{E}\left[\zeta_{p h}\right], \quad \lambda_{p p}=\mathcal{E}\left[\zeta_{p p}\right] .
\end{gathered}
$$

In the last line, we specialized to the $N=\infty$ expressions for $\Omega$ and $\Omega^{\prime}$ [Eq. (72)], which is valid for extracting the order $1 / N$ 


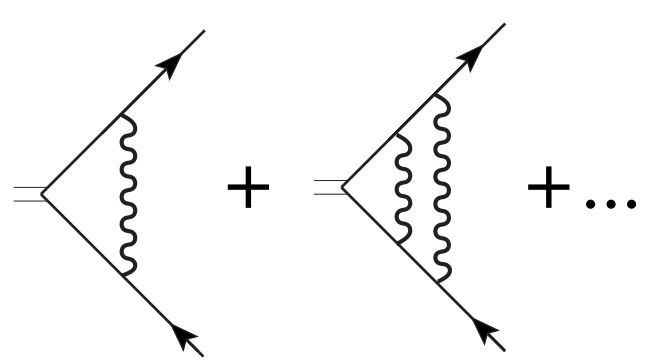

FIG. 11. Ladder diagrams that contribute to $V_{p h}$ at $1 / N$.

vertex corrections. Here, $\mathcal{E}[\zeta]$ is a dimensionless function of a dimensionless argument given by

$$
\begin{aligned}
\mathcal{E}[\zeta] & =\frac{3 \sqrt{3}}{2 \pi} \int_{0}^{\infty} \frac{t d t}{\left(1+t^{3}\right)\left(1+\zeta^{2} t^{4}\right)} \\
& =\frac{3 \pi \zeta^{5}+8 \pi\left(1-\zeta^{4}\right) / \sqrt{3}-3 \sqrt{2} \pi\left(\zeta^{1 / 2}-\zeta^{7 / 2}\right)-6 \zeta^{2} \ln \zeta}{8 \pi\left(1+\zeta^{6}\right) / \sqrt{3}}
\end{aligned}
$$

and has been normalized so that $\mathcal{E}[0]=1$. This function is monotonically decreasing with increasing argument, varying as $\mathcal{E}[\zeta] \approx \frac{3 \sqrt{3}}{8 \zeta}$ for $\zeta \rightarrow \infty$. The parameters $\zeta_{p h}$ and $\zeta_{p p}$ are given by

$$
\begin{gathered}
\zeta_{p h}=\frac{\Gamma^{2 / 3}\left(c+c^{\prime}\right)}{2 \chi^{2 / 3}\left(\frac{\Omega^{1 / 3}}{v}+\frac{\Omega^{\prime 1 / 3}}{v^{\prime}}\right)}, \\
\zeta_{p p}=\frac{\Gamma^{2 / 3} \mid c-c^{\prime}}{2 \chi^{2 / 3}\left(\frac{\Omega^{1 / 3}}{v}+\frac{\Omega^{\prime 1 / 3}}{v^{\prime}}\right)}, \\
\zeta_{p h}=\frac{\sqrt{3} \pi}{2 g^{2}} \Gamma\left(c+c^{\prime}\right), \quad \zeta_{p p}=\frac{\sqrt{3} \pi}{2 g^{2}} \Gamma \mid c-c^{\prime} .
\end{gathered}
$$

In the last line, we again used the $N=\infty$ values for $\Omega$ and $\Omega^{\prime}$. Notice that the vertex interaction strength between two fermions on opposing patches depends on their particular Fermi surface curvatures $c$ and $c^{\prime}$. Moreover, in the particle-particle channel the interaction strength is maximal when the curvatures are equal. This reflects a particle-particle nesting, which is responsible for the BCS pairing instability in the Fermiliquid context.

\section{Photon ladders}

Having evaluated the one-photon corrections $V^{(1 a)}$ to the bare vertices, we now discuss the full set of diagrams that appear at order $1 / N$ in each case. First, consider the fermion particle-hole vertex which enters into the density-density correlator: $V_{\rho_{\alpha}}^{(N)}=V_{p h ; \alpha, \alpha}^{(N)}$. The $1 / N$ diagrams are shown in Fig. 11 and contain an arbitrary number of noncrossing photon lines, ${ }^{42}$ beginning with the one-photon diagram $V_{p h}^{(1 a)}$ [Eq. (79)]. We shall denote the contribution from the diagram with $n$ photon lines as $V_{p h}^{(n a)}$. The magnitude of the singular contributions from each diagram in the ladder sum will depend on a dimensionless gauge charge,

$$
e_{\alpha}^{2} \equiv g^{2} / \Gamma c_{\alpha}
$$

Above, we found $V_{p h}^{(1 a)}=(1 / N) \mathcal{E}\left[\sqrt{3} \pi / e_{\alpha}^{2}\right] \ln \left(1 / q_{x}^{-}\right)$ $\equiv(1 / N) A_{1}^{(1)} \ln \left(1 / q_{x}^{-}\right)$. The singular contributions from the diagram with two-photon lines can also be extracted from the Feynman diagram, $\quad V_{p h}^{(2 a)}=(1 / N)\left[A_{1}^{(2)} \ln \left(1 / q_{x}^{-}\right)\right.$ $\left.+A_{2}^{(2)} \ln ^{2}\left(1 / q_{x}^{-}\right)\right]$, with $A_{1}^{(2)}$ and $A_{2}^{(2)}$ being universal dimensionless functions of $e_{\alpha}^{2}$. From this we infer the general structure for the diagram with $n$ photon lines,

$$
V_{p h}^{(n a)} \sim \frac{1}{N} \sum_{m=1}^{n} A_{m}^{(n)} \ln ^{m}\left(\frac{1}{q_{x}^{-}}\right) .
$$

This form suggests that the full set of $1 / N$ ladder diagrams can be exponentiated,

$$
V_{\rho_{\alpha}}^{(N)} \sim \frac{1}{N} \exp \left[\gamma_{\alpha} \ln \left(\frac{1}{q_{x}^{-}}\right)\right] \sim \frac{1}{N}\left|q_{x}-q_{x}^{\prime}\right|^{-\gamma_{\alpha}},
$$

with $\gamma_{\alpha}=\sum_{n=1}^{\infty} A_{1}^{(n)}$. The validity of this exponentiation can be justified by formally summing the ladder series to obtain an integral equation for the full vertex. The integral equation has a singular kernel and its solution is expected to have the power-law form with some exponent $\gamma_{\alpha}$, which will depend on the dimensionless charge $e_{\alpha}^{2}$.

Unfortunately, we have been unable to solve the integral equation to obtain $\gamma_{\alpha}$. However, we observed that for small charge $e_{\alpha}^{2}, A_{1}^{(1)}=3 e_{\alpha}^{2} /(8 \pi)+O\left(e_{\alpha}^{4}\right)$. This gives the leading behavior even though $A_{1}^{(1)}$ is not analytic in $e_{\alpha}^{2}$, as can be seen from Eq. (84); more generally, one finds $A_{m}^{(n)}=O\left(e_{\alpha}^{2 n}\right)$. Thus, assuming small parameter $e_{\alpha}^{2}$, the leading contribution to $\gamma_{\alpha}$ comes just from the single photon diagram,

$$
\gamma_{\alpha}=\frac{3}{8 \pi} e_{\alpha}^{2}+O\left(e_{\alpha}^{4}, \frac{1}{N}\right)
$$

For $e_{\alpha}^{2}$ of order 1, the exponent $\gamma_{\alpha}$, while obtained by summing diagrams at order $1 / N$, is itself of order 1 . It is the amplitude of the vertex in Eq. (90) which is of order $1 / N$. This reflects the particular structure of the large- $N$ theory, which, constructed to sum the nested rainbows for the fermion self-energy at leading order, also exponentiates the ladder sum for the particle-hole vertex at order $1 / N$. Notice that $\gamma_{\alpha}$ monotonically decreases as the curvature of the Fermi surface is taken large, due to the increasingly restrictive phase space requirements on the particle-hole vertex in this limit. We expect that this trend is valid more generally for larger $e_{\alpha}^{2}$ and for arbitrary $N$.

Summarizing the discussion of the particle-hole vertex, we can also write a scaling form for more general external momenta and frequencies,

$$
V_{\rho_{\alpha}}^{(N)} \sim\left|q_{x}-q_{x}^{\prime}\right|^{-\gamma_{\alpha}} \widetilde{V}_{\rho_{\alpha}}\left(\frac{q_{y}^{2}}{q_{x}^{-}}, \frac{q_{y}^{\prime 2}}{q_{x}^{-}}, \frac{\omega^{2 / 3}}{q_{x}^{-}}, \frac{\omega^{\prime 2 / 3}}{q_{x}^{-}}\right) .
$$

We next consider the vertex which enters in the bosonboson correlator, $V_{b}^{(N)}=V_{p p ; 1,2}^{(N)}$. The full set of diagrams that contribute to the particle-particle vertex are shown in Fig. 12 


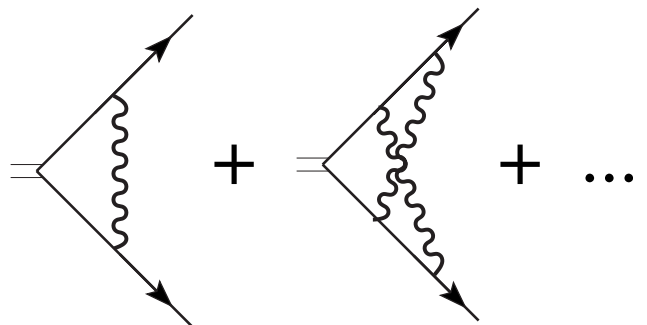

FIG. 12. Crossed diagrams that contribute to $V_{p p}$ at $1 / N$.

and contain an arbitrary number of maximally crossed photon lines. ${ }^{42}$ The first term is the one-photon diagram that we evaluated in Eq. (80), $\quad V_{p p ; 1,2}^{(1 a)}=(1 / N) \mathcal{E}\left[\zeta_{p p}\right] \ln \left(1 / q_{x}^{-}\right)$ $\equiv(1 / N) B_{1} \ln \left(1 / q_{x}^{-}\right)$(plus nonsingular contributions). An evaluation of the two-photon diagram gives the same form, $V_{p p ; 1,2}^{(2 a)}=(1 / N) B_{2} \ln \left(q_{x}^{-}\right)$. In contrast to the particle-hole case, here the two-photon diagram does not contain a log-squared term, which indicates that the crossed ladder series cannot be exponentiated. While we cannot evaluate the higher-order diagrams, we expect that each term will have a logarithmic contribution with some dimensionless amplitudes $B_{n}$. If this is the case, the full structure of the particle-particle vertex up to order $1 / N$ is

$$
V_{b}^{(N)}=1+\frac{\eta_{1} \ln \left(\Lambda_{x} / q_{x}^{-}\right)}{N}+O\left(\frac{1}{N^{2}}\right),
$$

with $\eta_{1}=\sum_{n=1}^{\infty} B_{n}$. To check whether the latter series can be defined in principle, we again take the limit of small dimensionless charges $e_{\alpha}^{2}$. Consider the least favorable case with matched Fermi surface curvatures, $c_{1}=c_{2}$, so $e_{1}^{2}=e_{2}^{2} \equiv e^{2}$ $=g^{2} /(\Gamma c)$. We find that the first term is independent of $e^{2}$, $B_{1}=1$. However, evaluating the two-photon diagram gives $B_{2} \sim\left(e^{2}\right)^{2} \ln ^{3}\left(1 / e^{2}\right)$ for small $e^{2}$. The diagrams are expected to be smaller when $c_{1} \neq c_{2}$ (for example, even the first term $B_{1}=\mathcal{E}\left[\zeta_{p p}\right]$ can be made small when $\left.g^{2} \ll \Gamma\left|c_{1}-c_{2}\right|\right)$. We thus conjecture that the series converges for small enough $e_{\alpha}^{2}$.

Provided it is legitimate to exchange the order of limits $N \rightarrow \infty$ and $q_{x} \rightarrow 0$ in Eq. (93), the boson vertex can be written in a power-law form,

$$
\left.V_{b}^{(N)}\left(\mathbf{q}, \omega ; \mathbf{q}^{\prime}, \omega^{\prime}\right)\right|_{q_{y}=q_{y}^{\prime}=\omega=\omega^{\prime}=0} \sim\left|q_{x}-q_{x}^{\prime}\right|^{-\eta},
$$

with

$$
\eta=\eta_{1} / N+O\left(1 / N^{2}\right) .
$$

We will discuss the legitimacy of this exponentiation procedure in Sec. VI. For more general external momenta and frequencies, the full boson vertex will satisfy a scaling form

$$
V_{b}^{(N)}\left(\mathbf{q}, \omega ; \mathbf{q}^{\prime}, \omega^{\prime}\right) \sim\left|q_{x}-q_{x}^{\prime}\right|^{-\eta} \widetilde{V}_{b}\left(\frac{q_{y}^{2}}{q_{x}^{-}}, \frac{q_{y}^{\prime 2}}{q_{x}^{-}}, \frac{\omega^{2 / 3}}{q_{x}^{-}}, \frac{\omega^{\prime 2 / 3}}{q_{x}^{-}}\right),
$$

where we have used the fact that the vertex depends only on the total $x$ momentum running through it $\left(q_{x}^{-}=q_{x}-q_{x}^{\prime}\right)$. The scaling function will vary as $\widetilde{V}_{b}(X, 0,0,0) \sim X^{-\eta}$ when any of its arguments $X \rightarrow \infty$. For example, one has $V_{b} \sim\left|q_{y}\right|^{-2 \eta}$ $\sim|\omega|^{-2 \eta / 3}$.

As will be detailed shortly, the singular vertices Eqs. (92) and (96) will lead to anomalous decay exponents for the boson density-density correlator and the boson Green's function.

\section{G. Boson and density correlators at finite $N$}

Since there are no singular $1 / N$ corrections to the fermion Green's functions, to extract the boson and density correlators up to order $1 / N$, one can use the $N=\infty$ fermion Green's functions when evaluating the bubble diagram. Using Eq. (71) and the scaling form Eq. (96) for the $V_{b}$ vertex enables the particle-particle bubble to be expressed as

$$
\Pi_{p p}^{(N)}(\mathbf{q}, \omega) \sim\left|q_{x}\right|^{-(2+\eta)} \int_{\mathbf{q}^{\prime} \omega^{\prime}} P\left(\frac{q_{y}^{2}}{q_{x}}, \frac{\omega^{2 / 3}}{q_{x}}, \frac{q_{x}^{\prime}}{q_{x}}, \frac{q_{y}^{\prime 2}}{q_{x}}, \frac{\omega^{\prime 2 / 3}}{q_{x}}\right),
$$

where $P$ is an appropriate scaling function. One can then scale out the $q_{x}$ dependence under the integrals to obtain

$$
\Pi_{p p}^{(N)}(\mathbf{q}, \omega) \sim\left|q_{x}\right|^{1-\eta} \tilde{\Pi}_{p p}\left(\frac{q_{y}^{2}}{q_{x}}, \frac{\omega^{2 / 3}}{q_{x}}\right) .
$$

We thereby arrive at the desired expression for the boson correlator,

$$
G_{b}^{(N)}\left(\mathbf{k}_{F_{1}}-\mathbf{k}_{F_{2}}+\mathbf{q}, \omega\right) \sim\left|q_{x}\right|^{1-\eta} \widetilde{G}\left(\frac{q_{y}^{2}}{q_{x}}, \frac{\omega^{2 / 3}}{q_{x}}\right),
$$

with exponent $\eta$ given in Eq. (95). While this result was obtained systematically to order $1 / N$, we expect it will remain valid more generally and, in particular, will involve the same scaling combinations $q_{y}^{2} / q_{x}$ and $\omega^{2 / 3} / q_{x}$. The scaling function $\widetilde{G}$ and the dependence of the exponent $\eta$ on the bare parameters such as the Fermi surface curvatures will presumably vary with $N$. Near the other momentum $\mathbf{k}_{F_{1}}+\mathbf{k}_{F_{2}}$, the boson correlator will satisfy a similar scaling form except with $\eta=0$. As argued before, we expect that the anomalous exponent at momentum $\mathbf{k}_{F_{1}}+\mathbf{k}_{F_{2}}$ will vanish in general, not just to leading order in $1 / N$.

The large-distance behavior of the equal-time boson Green's function in the DBL follows directly from Eq. (98) and was already listed in the Sec. I [Eq. (5)]. We remark that simple "power counting" using Eq. (98) works here because the relevant frequencies and momenta are indeed in the "scaling regime;" however, it should be kept in mind that we are dealing with line singularities and should exercise more caution with such arguments in general. Once again, both the Fermi wave vectors $\mathbf{k}_{F_{1}}$ and $\mathbf{k}_{F_{2}}$ and the scaling exponent $\eta$ will depend on the location and shape of the Fermi surface patches with normals along the observation direction $\hat{\mathbf{r}}$. At wave vector $\mathbf{k}_{F_{1}}-\mathbf{k}_{F_{2}}$, the Green's function decay becomes more slower due to the Amperean attraction between the $d_{1}$ and $d_{2}$ fermions. The fermions tend to move as pairs, but, unlike a Cooper pair, their motion is not phase coherent-the pairs are uncondensed. The boson correlator, while decaying more slowly with positive $\eta$, does not exhibit off-diagonal long-range order in the DBL phase. 
As we found to leading order in $1 / N$ [Eq. (95)], $\eta$ is expected to be largest when the two Fermi surface curvatures are equal. In the DBL phase which has closed Fermi surfaces, the characteristics of the $d_{1}$ and $d_{2}$ patches with normals along either diagonal $\hat{\mathbf{x}} \pm \hat{\mathbf{y}}$ are always equal due to the symmetry of the square lattice. Moreover, the decay of the boson Green's function $G_{b}(\mathbf{r})$ will be nonoscillatory along the diagonals,

$$
G_{b}(\mathbf{r}) \sim \frac{1}{|\mathbf{r}|^{4-\eta}}, \quad \hat{\mathbf{r}} \|(\hat{\mathbf{x}} \pm \hat{\mathbf{y}}),
$$

a prediction that we find consistent with the properties of the $(\text { det })_{x}(\text { det })_{y}$ wave functions studied in Sec. IV (see Fig. 7). For $c_{1}=c_{2}$, a very rough estimate can be extracted from the leading behavior, $\eta=(1 / N)+O\left(e^{2} / N\right)$ by setting $N=e^{2}=1$, giving $\eta \approx 1$. This would shift the $N=\infty$ decay exponent back to its mean field value of $1 /|\mathbf{r}|^{3}$. As noted earlier, the gauge fluctuations encapsulated already at $N=\infty$ strongly modify the motion of each fermion, leading to a boson that moves less coherently, decaying with a larger exponent than in the mean field theory. The Amperean attraction between the pair of fermions which enters at order $1 / N$ compensates this effect, leading to a slower decay of the boson correlator. For $\eta=1$, the two competing effects exactly compensate one another.

Next we consider the density-density correlator. Just as for the boson correlator, the density correlator will satisfy a scaling form:

$$
D_{d_{\alpha}}^{(N)}\left(2 \mathbf{k}_{F_{\alpha}}+\mathbf{q}, \omega\right) \sim\left|q_{x}\right|^{1-\gamma_{\alpha}} \tilde{D}_{2 \mathbf{k}_{F_{\alpha}}}\left(\frac{q_{y}^{2}}{q_{x}}, \frac{\omega^{2 / 3}}{q_{x}}\right),
$$

with an anomalous exponent that depends on the Fermi surface patches [see Eq. (91)]. Back in real space, the dominant density correlator oscillates with wave vector $2 \mathbf{k}_{F_{\alpha}}$, with an envelope decaying as a power law with the anomalous exponent,

$$
D_{d}^{(N)}(\mathbf{r}) \sim-\frac{\cos \left[2 \mathbf{k}_{F_{\alpha}} \cdot \mathbf{r}-3 \pi / 2\right]}{|\mathbf{r}|^{4-\gamma_{\alpha}}}-\frac{1}{|\mathbf{r}|^{4}} .
$$

We have also indicated that the zero-momentum component is not modified relative to the $N=\infty$ behavior. Again, both $\mathbf{k}_{F_{\alpha}}$ and $\gamma_{\alpha}$ are particular to the Fermi surface patches with normals parallel to $\pm \hat{\mathbf{r}}$. In contrast to the particle-particle channel, the particle-hole channel is not nested so that we suspect the exponents $\gamma_{\alpha}$ will be smaller than $\eta$ for the physically relevant case with $N=1$.

In sum, at leading order in our systematic $1 / N$ expansion, the boson correlator was found to exhibit power-law decay with an exponent that depends on the bare Fermi surface curvatures, varying continuously around the Fermi surface. Similar behavior was found for the density-density correlator. Provided this qualitative behavior persists down to the physically relevant case of $N=1$, we conclude that the DBL phase is described by a manifold of scale invariant theories rather than an isolated fixed point. Within the $1 / N$ expansion, the power-law form obtained for the boson correlator relied on the exponentiation of the logarithmic behav- ior on momentum and frequency of the leading $1 / N$ correction. In the next section, we check the legitimacy of this procedure by revisiting the renormalization group approach developed earlier to describe the low-energy physics of a sea of fermions coupled to a U(1) gauge field.

We conclude this section by pointing out that throughout we worked on the assumption of having both $d_{1}$ and $d_{2}$ parallel patches present, as in Fig. 10. In the case with open Fermi surfaces, shown in the right panel of Fig. 1, we encounter situations when for a given observation direction $\hat{\mathbf{r}}$ only one Fermi surface (or even none at all) has patches with normals in this direction. In such cases, the boson correlator will decay exponentially; there is no interspecies gauge interactions (one species is simply absent), but the fermions that are present are still strongly affected by the gauge field, and in particular, the preceding analysis of their density correlations remains the same. In the DLBL phase, there are no parallel patches on the entire two Fermi surfaces, so the $d_{1}$ and $d_{2}$ fermions effectively decouple from each other. It is also interesting to remark that in the limit of extreme $d$ eccentricity when the Fermi surfaces are completely flat, we expect that the relevant gauge field is very strongly damped. This can be seen, e.g., from the naive RPA for the Landau damping coefficient $\Gamma$ [Eq. (55)] in the limit of vanishing curvatures. In this limit, the energy scale $\Omega$ [Eq. (72)] below which fermions become incoherent goes to zero, which leads us to speculate that perhaps in this case the $d_{1}$ and $d_{2}$ fermions behave as essentially free.

\section{EFFECTIVE FIELD THEORY FOR THE DBL: CRITICALITY AND STABILITY}

Polchinski ${ }^{36}$ and others ${ }^{37,38}$ argued that $2 \mathrm{D}$ fermions interacting with a U(1) gauge field can be fruitfully studied within a renormalization group analysis of a particular effective field theory. In the present case, the fixed-point theory has an action given by

$$
S_{\text {fixed point }}=S_{d}+S_{a}+S_{i n t},
$$

with

$$
\begin{aligned}
S_{d}= & \sum_{\alpha s} \int_{\mathbf{q} \omega} d_{\alpha s}^{\dagger}(\mathbf{q}, \omega)\left(-i \omega\left|\frac{\Omega_{\alpha}}{\omega}\right|^{1 / 3}+s v_{\alpha} q_{x}\right. \\
& \left.+\frac{v_{\alpha} c_{\alpha}}{2} q_{y}^{2}\right) d_{\alpha s}(\mathbf{q}, \omega),
\end{aligned}
$$

while the gauge field part $S_{a}$ is given in Eq. (54) and the fermion-gauge coupling is specified in Eq. (53). Within this fixed point Ansatz, both the gauge field and the fermions have singular propagators, but their interaction is local. The advantage of this effective field theory is that it is possible to define a simple renormalization group transformation which leaves the Gaussian part of the theory invariant and under which the interaction strength $g$ is a marginal perturbation. If $g$ is assumed to be small, it can be treated via a conventional perturbative renormalization group (RG) approach as we describe below. One finds that the full effective field theory is invariant under the RG; i.e., it is at a fixed point. This 
strongly suggests that exponentiating the logarithmic singularities present in the large- $N$ approach, as we did to order $1 / N$ above, is a correct procedure at all orders in $1 / N$. If it is, then at order $1 / N^{2}$ one must find $(\log )^{2}$ singularities with particular coefficients such that the exponentiation procedure to obtain a power-law behavior is consistent.

An unsettling drawback with this effective field theory approach is that it leaves unclear what constraints must be placed on additional interactions that can be added to the theory. At the very least, these interactions must be gauge invariant and consistent with momentum conservation, but is it legitimate to require that the interactions be local in the fermion fields? For example, can one require that fourfermion interactions be local with nonsingular coefficients, or will singular interactions necessarily be generated? Indeed, if one were to formally integrate out the gauge field with its singular propagator, one would generate fourfermion interactions with a particular singular form. In what follows, we will ignore these subtleties, exploring the possible perturbative instabilities driven by nonsingular fermion interactions. Specifically, we will consider all nonsingular quartic interactions involving four fermions living near the Fermi surfaces that are consistent with the relevant symmetries, most importantly, momentum conservation.

The fixed-point action, as it stands, has nine parameters:

$$
\Gamma, \chi, v_{\alpha}, c_{\alpha}, \Omega_{\alpha}, g \text {. }
$$

However, with an appropriate rescaling of the fermion fields and the gauge field together with the momenta and frequency, it is possible to set five of these parameters to unity: $\widetilde{\Gamma}=\widetilde{\chi}=\widetilde{g}=\widetilde{v}_{\alpha}=1$. The effective field theory is then specified by four dimensionless parameters: $\tilde{c}_{\alpha}=c_{\alpha} \Gamma / g^{2}$ and $\widetilde{\Omega}_{\alpha}$ $=\Omega_{\alpha} \chi^{2} \Gamma /\left(g^{2} v_{\alpha}^{3}\right)$. Evidently, this theory is not describing a fixed "point" per se, but constitutes a four-dimensional manifold of theories which are invariant under the RG. Establishing definitively that a particular bare (lattice) gauge theory Hamiltonian is attracted to this manifold is exceedingly difficult. Arguably, it is even harder to deduce where on this manifold the theory flows. The values of the dimensionless parameters which are obtained from the leading large- $N$ analysis can, nevertheless, be used as a rough guide in addressing both questions.

The RG analysis of the fermion-gauge action, $S_{\text {fixed point }}$, proceeds as follows. At each stage, the fermion fields reside in the appropriate momentum space patches $\left|q_{x}\right|<\Lambda$ and $\left|q_{y}\right|<(\Lambda / c)^{1 / 2}$, where $\Lambda$ is the shell width in the direction normal to the Fermi surface. The corresponding restriction on the frequency is $|\omega|<(v \Lambda)^{3 / 2} / \Omega^{1 / 2}$. The gauge fields reside in similar momentum-frequency regions but centered around zero momentum, and the overall setup is illustrated in Fig. 10. If the dimensionless parameters exhibited earlier are of order 1 (which is the case if the parameters are taken from the large- $N$ analysis), the corresponding regions are roughly similar for all fermion and gauge fields. Also, in practical RG calculations beyond the tree level, it is convenient to keep the cutoff only on the frequencies and perform unrestricted integrations over the momenta.
We integrate out the high-energy fields from the shell between $\Lambda$ and $\Lambda / b$, and then rescale the momenta and frequencies in order to recover the initial cutoff: $q_{x}=q_{x}^{\prime} / b, q_{y}$ $=q_{y}^{\prime} / b^{1 / 2}$, and $\omega=\omega^{\prime} / b^{3 / 2}$. We also perform appropriate rescaling of the fields: $d=b^{2} d^{\prime}$ and $a=b^{2} a^{\prime}$. Upon such treelevel scaling, the fixed-point action remains exactly as before; i.e., all couplings remain unchanged. It is also useful to bear in mind that even though we nominally restore all cutoffs as we proceed, in terms of the original momenta the patches become more and more elongated in the $\hat{\mathbf{y}}$ direction in Fig. 10. In particular, any overlap between two patches that correspond to nearby but nonparallel tangents $\hat{\mathbf{y}}$ and $\hat{\mathbf{y}}^{\prime}$ goes to zero in the low-energy limit.

To proceed with the analysis beyond the tree level, we will work perturbatively in the (dimensionless) fermiongauge coupling, assuming that it is small. Quite generally, since the momentum shell RG cannot produce terms that are singular at small frequencies and momenta, the couplings $\Omega_{\alpha}$ and $\Gamma$ will not renormalize at any order in the perturbation expansion. On the other hand, the parameters $v_{\alpha}, c_{\alpha}, \chi$, and $g$ can potentially flow. However, a direct examination shows that they do not renormalize at the lowest (one loop) order, and we strongly suspect that this remains true at all orders. The implication is that the fermion-gauge interaction is exactly marginal and that $S_{\text {fixed point }}$ describes a manifold of RG fixed points parametrized by four dimensionless couplings. Although the fermion-gauge coupling need not be small, we expect that this RG-invariant manifold will extend outside the perturbatively accessible regime. This manifold describes the putative DBL phase.

In order to establish the stability of the DBL, however, we need to consider the effects of all symmetry allowed perturbations that can be added to $S_{\text {fixed point }}$. Stability requires that all such perturbations are irrelevant under the RG. Restricting ourselves to local terms, we focus now on the fourfermion interactions which are most likely to destabilize the fixed-point manifold.

\section{A. Short-range fermion interactions}

Let us consider a general quartic term,

$$
W d_{\alpha_{1} s_{1}}^{\dagger} d_{\alpha_{2} s_{2}}^{\dagger} d_{\alpha_{3} s_{3}} d_{\alpha_{4} s_{4}},
$$

which can contain different fermion species labeled by $\alpha$ and involve different patches labeled by $s$. The fermion-patch labels are assumed such as to satisfy the momentum conservation. Also, we do not show explicitly the (conserved) frequency and momentum of the fermion fields. The amplitude for this four-fermion interaction, $W$, measures the strength of a direct scattering between two fermions.

At the tree level in the RG, all such short-range interactions are irrelevant,

$$
W \rightarrow W^{\prime}=W / b,
$$

scaling toward zero at low energies. Physically, the incoherent motion of the fermions, manifest in their $\omega^{2 / 3}$ self-energy, leads to a reduced phase space for direct two-body interactions relative to the case for free fermions. 


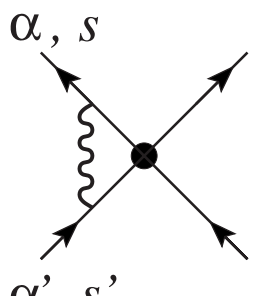

a)

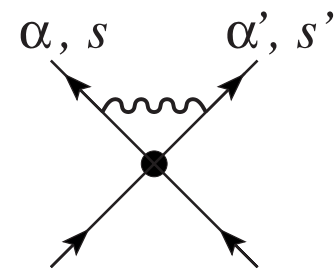

b)
FIG. 13. One-loop diagrams needed to calculate renormalization of four-fermion interactions.

At one loop, a gauge propagator can connect any two legs of the quartic vertex, which involve fermions located on patches with parallel or antiparallel Fermi surface normals. Two such physically distinct diagrams are shown in Fig. 13. Both processes correspond to an interaction between the two fermions mediated by the Landau damped gauge field. The one-loop integrals needed to evaluate these diagrams are identical to those for the vertex corrections in Sec. V F 1, and give the following contributions to the four-fermion interaction before any rescaling:

$$
\begin{gathered}
\delta W=\operatorname{sign}\left(g_{\alpha} g_{\alpha^{\prime}}\right) \delta_{s,-s^{\prime}} \lambda_{p h} W \ln (b), \\
\delta W=-\operatorname{sign}\left(g_{\alpha} g_{\alpha^{\prime}}\right) \delta_{s,-s^{\prime}} \lambda_{p p} W \ln (b) .
\end{gathered}
$$

Here, the primed and unprimed parameters refer to the possibly distinct species of the two fermions connected by the gauge propagator. The anomalous exponents $\lambda_{p h}$ and $\lambda_{p p}$ are given in Eqs. (81) and (82) - these formulas are general and do not assume any relations among the parameters, as appropriate in the present RG setup. Sometimes, which will be indicated explicitly, we will use the $N=\infty$ expressions [Eq. (83)] to get a crude estimate of the numerical values.

Similar to the analysis in Sec. V F 1, either diagram gives zero if the fermions involved have parallel group velocities, whereas a nontrivial contribution is found when the two fermion fields reside on opposite patches with antiparallel group velocities. The sign is again determined by Ampere's law: Two fermions emerging from a scattering process with parallel charge currents attract one another, spend more time close together, and consequently enhance the amplitude for the scattering strength $W$. Conversely, the interaction strength is suppressed when the pair of scattered fermions have antiparallel charge currents. The difference in the absolute values of the contributions from Figs. 13(a) and 13(b) is similar to the distinction between the particle-hole [chargedensity wave (CDW)] and particle-particle (BCS) processes in a Fermi liquid. Thus, in the situation when $c$ and $c^{\prime}$ have the same sign, the particle-hole process is not nested because of the curvature of the Fermi surface. On the other hand, the particle-particle process is somewhat better nested and becomes perfectly nested when $c=c^{\prime}$, which is the familiar phase space reasoning in the theory of the BCS instability. This distinction between the processes can be clearly seen from the arguments $\zeta_{p h}$ and $\zeta_{p p}$ [Eqs. (85) and (86)] of the function $\mathcal{E}[\zeta]$ in the two cases.
With the above general expressions in hand, we now consider specific four-fermion interactions. Of interest is the one-loop contribution $Y \propto g^{2}$ to the eigenvalue of such perturbations at the fixed point,

$$
\frac{d W}{d l}=(-1+\Upsilon) W+O\left(W^{2}\right) .
$$

The $O\left(W^{2}\right)$ terms, which, in general, mix different fourfermion terms, can be deduced similarly to the RG treatment of quartic interactions around the Fermi-liquid fixed point $^{43,44}$ and, in fact, have very similar structure. In the Fermi-liquid case, the interactions are marginal and there is no term linear in $W$, so the $O\left(W^{2}\right)$ terms determine the physics. In the fermion-gauge case, on the other hand, the interactions can be either relevant when $Y>1$ or irrelevant when $\Upsilon<1$. For $Y<1$, the DBL phase is stable to weak perturbations, but sufficiently strong bare interactions might still drive the DBL through a quantum phase transition into another phase. In this case, the $O\left(W^{2}\right)$ terms could be helpful in discerning the nature of the new phase.

As in Fermi-liquid theory, the strongest constraint on the allowed quartic interactions comes from momentum conservation, since all four fermions have to live near the Fermi surfaces. In Fermi-liquid theory, there are only two allowed types of vertices, the forward scattering interactions, which contribute to the Landau parameters, and the Cooper vertices. We first consider interactions in which all four fermions are of the same species. A general forward scattering interaction then takes the form

$$
W_{\mathbf{k}, \mathbf{k}^{\prime}}^{f} d_{\mathbf{k} \alpha}^{\dagger} d_{\mathbf{k} \alpha} d_{\mathbf{k}^{\prime} \alpha}^{\dagger} d_{\mathbf{k}^{\prime} \alpha}
$$

where $\mathbf{k}, \mathbf{k}^{\prime}$ are two wave vectors on the species $\alpha$ Fermi surface. Consider first the generic case with $\mathbf{k} \neq \pm \mathbf{k}^{\prime}$. Since this interaction does not involve opposite patches on the Fermi surface, the one-loop contribution will vanish, $Y^{f}=0$. This is also the case when $\mathbf{k}=\mathbf{k}^{\prime}$. The forward scattering vertex with $\mathbf{k}=-\mathbf{k}^{\prime}$ is part of the Cooper channel to which we now turn.

For a single species of fermion, there exists only the odd angular momentum Cooper pairing channel ("triplet pairing"),

$$
W_{\mathbf{k}, \mathbf{k}^{\prime}}^{t} d_{\mathbf{k} \alpha}^{\dagger} d_{-\mathbf{k} \alpha}^{\dagger} d_{-\mathbf{k}^{\prime} \alpha} d_{\mathbf{k}^{\prime} \alpha}
$$

with $W_{\mathbf{k}, \mathbf{k}^{\prime}}^{t}=-W_{-\mathbf{k}, \mathbf{k}^{\prime}}^{t}=-W_{\mathbf{k},-\mathbf{k}^{\prime}}^{t}$. The one-loop contribution is negative, $Y_{\mathbf{k}, \mathbf{k}^{\prime}}^{t}=-\left(\lambda_{p p}^{\mathbf{k}}+\lambda_{p p}^{\mathbf{k}^{\prime}}\right)$ for $\mathbf{k} \neq \pm \mathbf{k}^{\prime}$, due to the Amperean repulsion between the two particles (and between the two holes) with antiparallel group velocities. For the special case $\mathbf{k}= \pm \mathbf{k}^{\prime}$, one has $\Upsilon_{\mathbf{k}, \pm \mathbf{k}}^{t}=-2\left(\lambda_{p p}^{\mathbf{k}}-\lambda_{p h}^{\mathbf{k}}\right)$ since there is now also an Amperean attraction between particles and holes on opposite patches of the Fermi surface. However, since we expect $\lambda_{p p}^{\mathbf{k}} \geqslant \lambda_{p h}^{\mathbf{k}}$ due to the nesting in the particle-particle channel, one has $Y^{t} \leqslant 0$ in this case as well. Thus, the oneloop contribution makes this triplet pairing vertex more irrelevant. Physically, the repulsive gauge interaction between two $d_{\alpha}$ fermions is unfavorable for their pairing.

We now turn to vertices which involve two $d_{1}$ fermions and two $d_{2}$ fermions. For simplicity, we first focus on the 
situation with vanishing $d$ eccentricity, so that the two Fermi surfaces coincide. Consider first the forward scattering interactions coupling the densities of the two fermion species,

$$
W_{\mathbf{k}, \mathbf{k}^{\prime}}^{f ; 1,2} d_{\mathbf{k} 1}^{\dagger} d_{\mathbf{k} 1} d_{\mathbf{k}^{\prime} 2}^{\dagger} d_{\mathbf{k}^{\prime} 2} \text {. }
$$

The only nonvanishing one-loop correction is when $\mathbf{k}=-\mathbf{k}^{\prime}$, and gives $Y_{\mathbf{k},-\mathbf{k}}^{f ; 1,2}=2\left(\lambda_{p p}^{\mathbf{k}}-\lambda_{p h}^{\mathbf{k}}\right) \geqslant 0$. If this is greater than 1 , it would signal a possible instability, but in any event, we will see that this is smaller than the eigenvalue in the conventional BCS "singlet pairing" channel that we consider next.

The singlet BCS interaction is of the form

$$
W_{\mathbf{k}, \mathbf{k}^{\prime}}^{s} d_{\mathbf{k} 1}^{\dagger} d_{-\mathbf{k} 2}^{\dagger} d_{-\mathbf{k}^{\prime} 2} d_{\mathbf{k}^{\prime} 1} \text {. }
$$

With zero $d$ eccentricity as assumed here, the BCS interaction is nested. Moreover, since the two particles (or the two holes) here carry opposite gauge charge and, therefore, have parallel gauge currents, there will be an Amperean attraction giving a positive contribution to the Cooper vertex: $Y_{\mathbf{k}, \mathbf{k}^{\prime}}^{s}$ $=\lambda_{p p}^{\mathbf{k}}+\lambda_{p p}^{\mathbf{k}^{\prime}}$ for $\mathbf{k} \neq \pm \mathbf{k}^{\prime}$. For a circular Fermi surface, symmetry dictates that $\lambda_{p p}^{\mathbf{k}}$ will be $\mathbf{k}$ independent, but this is actually the case, generally, for such one-loop contribution in the case with matched $d_{1}$ and $d_{2}$ Fermi surfaces, since the curvatures on the opposing patches are equal. Thus, as claimed earlier, the eigenvalue of the singlet BCS pairing interaction, $\Upsilon^{s}$ $=2 \lambda_{p p}$, is larger than the corresponding eigenvalue of the forward scattering interaction coupling the densities of the two species on opposite sides of the Fermi surface, $Y^{s}$ $>Y_{\mathbf{k},-\mathbf{k}}^{f ; 1,2}$. For the special case $\mathbf{k}=-\mathbf{k}^{\prime}$, there will be an additional positive contribution to the eigenvalue of the singlet BCS pairing interaction due to the Amperean attraction between particles and holes, $\Upsilon_{\mathbf{k},-\mathbf{k}}^{s}=2\left(\lambda_{p p}^{\mathbf{k}}+\lambda_{p h}^{\mathbf{k}}\right)$. However, being a set of measure zero, this will not contribute to a BCStype pairing instability. At the present, it is not clear what state would be preferred by a large interaction of this form and what significance this might have.

It is interesting to use the $N=\infty$ parameters to deduce an approximate value for the one-loop contribution to $Y^{s}$. With zero $d$ eccentricity, the two Fermi surfaces coincide, so the curvatures are equal, $c_{1}=c_{2}$, and $\lambda_{p p}^{\mathbf{k}}=\mathcal{E}[0]=1$ for all $\mathbf{k}$. This gives $Y^{s}=2$ and implies that the BCS interaction is strongly relevant,

$$
\frac{d W^{s}}{d l}=\left(-1+\Upsilon^{s}\right) W^{s}=W^{s},
$$

within this approximation. Since we expect some bare shortrange attraction between the $d_{1}$ and $d_{2}$ particles, the runaway flows will lead to BCS pairing. The composite boson $b^{\dagger}$ $=d_{1}^{\dagger} d_{2}^{\dagger}$ will condense, resulting in a superfluid phase. Thus, based on this analysis, we suspect that the putative $S$-type Bose liquid phase accessed from the gauge theory with identical Fermi surfaces for $d_{1}$ and $d_{2}$ will be generically unstable toward superfluidity.

We now consider the situation with nonzero $d$ eccentricity. In this case, $d_{1}$ and $d_{2}$ have different Fermi surfaces and the Cooper vertex will no longer be nested. This precludes a conventional weak coupling BCS instability for the $d$-wave Bose liquid phase. The remaining channels that could poten- tially drive a weak coupling instability are the forward scattering interactions given in Eq. (111), with the locations $\mathbf{k}$ and $\mathbf{k}^{\prime}$ on the two Fermi surfaces tuned to have antiparallel patch normals.

For bosons on the square lattice, with increasing $d$ eccentricity, the two closed Fermi surfaces will eventually open up and, above some critical eccentricity, the requirement of antiparallel $d_{1}$ and $d_{2}$ Fermi surface normals will no longer be possible. In this large $d$-eccentricity regime, when the two Fermi surfaces are so dissimilar, there are no such forward scattering interactions coupling the two species which can be enhanced by the gauge fluctuations. Sometimes one can find interactions that can be enhanced via the particle-hole attraction within the same species, e.g., $d_{\mathbf{k} 1}^{\dagger} d_{-\mathbf{k} 1} d_{\mathbf{k}^{\prime} 2}^{\dagger} d_{\mathbf{k}^{\prime \prime} 2}$, which require tuning both $\mathbf{k}^{\prime}$ and $\mathbf{k}^{\prime \prime}$ to satisfy momentum conservation, but this is not always possible. In any event, the corresponding one-loop $Y=\lambda_{p h}^{\mathbf{k}}$ is not likely to change the irrelevance of this term from the tree level; e.g., the $N=\infty$ estimate of $\lambda_{p h}^{\mathbf{k}}$, [Eq. (83)], is always smaller than 1. Regarding other interactions, none of the intraspecies four-fermion terms have positive eigenvalues. We conclude that in this large $d$-eccentricity regime, the $d$-wave local Bose liquid (as specified in Sec. III C) will exist as a stable phase.

Returning to the case with smaller $d$ eccentricity, the stability of the DBL phase will depend on the eigenvalues $\Upsilon^{f}(\hat{\mathbf{n}})$ of the forward scattering interaction that couples a patch on one Fermi surface with normal $\hat{\mathbf{n}}$ to a patch on the other Fermi surface with antiparallel normal. The particles on opposing patches experience a strong Amperean attraction, whereas a particle and hole experience a weaker repulsion, so that the eigenvalues $\Upsilon^{f}(\hat{\mathbf{n}})=2\left(\lambda_{p p}-\lambda_{p h}\right)$ will be positive for all orientations of $\hat{\mathbf{n}}$. However, the magnitude of $\Upsilon^{f}(\hat{\mathbf{n}})$ depends on the Fermi surface curvatures and will vary with orientation. This can be made explicit by evaluating the one-loop contribution using the $N=\infty$ values of the various parameters; thereby we obtain an approximate expression for the eigenvalue,

$$
Y^{f}(\hat{\mathbf{n}}) \approx 2 \mathcal{E}\left[\frac{\sqrt{3} \pi}{2 g^{2}} \Gamma\left|c_{1}-c_{2}\right|\right]-2 \mathcal{E}\left[\frac{\sqrt{3} \pi}{2 g^{2}} \Gamma\left(c_{1}+c_{2}\right)\right] .
$$

The first contribution is maximal and equal to 2 when the curvatures on the two Fermi surfaces coincide, which they will when $\hat{\mathbf{n}}$ is along a diagonal of the square lattice. We have no such estimate of the second contribution since $g, \Gamma$, and $c_{\alpha}$ are independent in our $N=\infty$ theory; if we use in addition, a crude RPA [Eq. (55)], we get the total $\Upsilon^{f}=2$ $-2 \mathcal{E}[\sqrt{3}]=1.53>1$. In this case, the $d$-wave Bose liquid in this regime of $d$ eccentricity will presumably be unstable, driven by the forward scattering interactions with $\hat{\mathbf{n}}=\hat{\mathbf{x}} \pm \hat{\mathbf{y}}$ along the diagonals of the square lattice.

The above numerical estimate is very uncontrolled and is given only to see possible trends. Still, let us speculate what the resulting phase might be if there is, indeed, such instability. Most naively, there are two guesses, obtained by a mean field decoupling of the four-fermion interaction $d_{\mathbf{k} 1}^{\dagger} d_{\mathbf{k} 1} d_{\mathbf{k}^{\prime} 2}^{\dagger} d_{\mathbf{k}^{\prime} 2}$, where $\mathbf{k}$ and $\mathbf{k}^{\prime}$ are the locations of the two patches with normals $\hat{\mathbf{n}}$ and $-\hat{\mathbf{n}}$ that give the largest $\Upsilon^{f}$. We can assume a nonvanishing particle-particle or particle-hole 
condensate, $\left\langle d_{\mathbf{k}_{1} 1}^{\dagger} d_{\mathbf{k}^{\prime} 2}^{\dagger}\right\rangle$ or $\left\langle d_{\mathbf{k}_{1}}^{\dagger} d_{\mathbf{k}^{\prime} 2}\right\rangle$, respectively. Both condensates carry nonzero momentum, $\mathbf{k}+\mathbf{k}^{\prime}$ for the particleparticle condensate and $\mathbf{k}-\mathbf{k}^{\prime}$ for the particle-hole condensate. However, since the interaction is repulsive in the particle-hole channel, such a condensate seems rather unlikely. Also, the combination $d_{1}^{\dagger} d_{2}$ carries a nonzero gauge charge, so is unphysical and cannot serve as a legitimate order parameter [but the product $\left\langle d_{\mathbf{k}_{1} 1}^{\dagger} d_{\mathbf{k}^{\prime} 2} d_{-\mathbf{k}^{\prime} 2}^{\dagger} d_{-\mathbf{k} 1}\right\rangle$ is gauge invariant, and if condensed, would correspond to an energy density wave at momentum $\left.2\left(\mathbf{k}-\mathbf{k}^{\prime}\right)\right]$.

If there is an instability, we think it is more likely to occur in the particle-particle channel. The order parameter $\left\langle d_{\mathbf{k}^{1} 1}^{\dagger} d_{\mathbf{k}^{\prime} 2}^{\dagger}\right\rangle$ is gauge invariant and corresponds physically to a finite momentum Bose condensate. The situation is analogous to the Fulde-Ferrell-Larkin-Ovchinnikov problem, and as there, provided the condensate is not too strong, it will only gap out parts of the Fermi surfaces. As such, this would still be a very unusual type of superfluid, which in a mean field description would have residual gapless fermionic excitations. Beyond the mean field, the gauge fluctuations would still scatter the gapless fermions, rendering them incoherent. This state would thus correspond to finite momentum Bose condensation coexisting with the DBL.

In sum, whether or not the DBL for small $d$ eccentricity is unstable to such finite momentum pairing is a quantitative issue which will depend on the values of the anomalous dimensions. An analysis of the actual wave functions in Sec. IV reveals no such tendencies, and so might be taken as an indication against the instability. Indeed, as we saw in Sec. IV, the $S$-wave Bose liquid wave function does appear generically unstable towards a conventional superfluid, consistent with the expectation of a zero-momentum BCS instability in the gauge theory. In the same spirit, we will see in the next section that for hard-core bosons at half filling, the DBL wave function in the extreme $d$-eccentricity limit (corresponding to fermions which can only hop in one of the two directions on the square lattice) reveals a coexistence of a commensurate $(\pi, \pi)$ CDW with a gapless DBL Fermi surface. This indicates that the Gutzwiller wave functions for the DBL, at least in some special instances, can also reveal translational symmetry breaking instabilities, while we repeat that no such instabilities are observed for generic DBL wave functions.

This concludes our discussion of the effective field theory description and possible instabilities of the DBL phase. As we have seen, the main potential instability involves pairing $d_{1}$ and $d_{2}$ fermions moving with opposite group velocities, but such BCS channel is suppressed for mismatched Fermi surfaces and is completely eliminated in the DLBL regime, which appears to be particularly stable.

\section{RING HAMILTONIAN ENERGETICS FOR THE DBL PHASE}

In this section, we ask what energetics may stabilize the DBL or DLBL phases. Specifically, we motivate and study the $J-K_{4}$ Hamiltonian [Eq. (8)] with competing boson hopping and ring exchange terms. When $J>0$ and $K_{4}>0$, this
Hamiltonian does not satisfy the Marshall sign conditions, so one expects the ground state wave function to take both positive and negative values.

The Hamiltonian (8) is motivated by considering the gauge theory description [Eq. (17)] of the DBL phase in the strong coupling limit of the gauge theory, $h \gg K, t_{\|}, t_{\perp}$. In this limit, one can perturbatively eliminate the gauge field; the resulting boson Hamiltonian contains, among other terms, both $H_{J}$ and $H_{4}$ with $J=A t_{\|} t_{\perp} / h$ and $K_{4}=A^{\prime} K t_{\|}^{4} / h^{4}$ $-A^{\prime \prime} t_{\|}^{2} t_{\perp}^{2} / h^{3}$, with positive numerical coefficients $A, A^{\prime}$, and $A^{\prime \prime}$. Only the signs of the contributions to $J$ and $K_{4}$ are of interest here, since, in what follows, both $J$ and $K_{4}$ are taken as free parameters of the Hamiltonian [Eq. (8)]. Coming from the gauge theory, we have $J>0$ and $K_{4}>0$ in the regime of primary interest when $K \sim h$ and $t_{\|} \gg t_{\perp}$. To define the system, we also need to specify the boson density per site $\rho$. The hard-core boson model has particle-hole symmetry, so it is enough to consider $\rho \leqslant 1 / 2$.

Before proceeding, we note that the above Hamiltonian with $J>0$ and $K_{4}<0$ was introduced and analyzed in Ref. 14. Since there is no sign problem in this case, extensive quantum Monte Carlo studies of Refs. 15-17 were able to map out the phase diagram of the model. In addition to a bosonic superfluid phase, the numerical studies found two ordered phases at half filling, a bond-ordered stripe phase and a staggered CDW. When the Monte Carlo was performed at fixed chemical potential, ${ }^{16}$ varying the chemical potential drove first-order transitions out of the commensurate solid phases into the superfluid. On the other hand, when the Monte Carlo was performed at fixed density, ${ }^{17}$ the superfluid phase was observed as soon as the density dropped below half filling, while a phase separation occurred when the density was decreased further.

The case $J>0$ and $K_{4}>0$ was not studied in Monte Carlo since there is a sign problem. Below, we present a rudimentary variational energetics study of this regime. The results are summarized in Fig. 15 and lend some support that the DLBL phase of Sec. III C may be stabilized by such competing hopping and ring exchange terms.

\section{A. Model with ring exchanges only}

Consider first the Hamiltonian with the ring term only. In this case, we can change the sign of $K_{4}$ by dividing the square lattice into four sublattices and performing a transformation $b \rightarrow-b$ on one sublattice. The cited quantum Monte Carlo studies did not consider the pure ring model, but extrapolating these to $J=0$, it seems likely that at half filling the ground state is the $(\pi, \pi) \mathrm{CDW}$. However, we also expect that there is a regime away from half filling that realizes a novel EBL phase discovered and studied by Paramekanti et al. ${ }^{14}$ which we now discuss.

The EBL was accessed by considering a rotor version of the ring Hamiltonian,

$$
\begin{aligned}
H_{\text {rotor }}= & \frac{\mathcal{U}}{2} \sum_{\mathbf{r}}\left(n_{\mathbf{r}}-\bar{n}\right)^{2}-|\mathcal{K}| \sum_{\mathbf{r}} \cos \left(\phi_{\mathbf{r}}-\phi_{\mathbf{r}+\hat{\mathbf{x}}}+\phi_{\mathbf{r}+\hat{\mathbf{x}}+\hat{\mathbf{y}}}\right. \\
& \left.-\phi_{\mathbf{r}+\hat{\mathbf{y}}}\right),
\end{aligned}
$$

where the phase $\phi_{\mathbf{r}}$ and the boson density $n_{\mathbf{r}}$ are canonically 
conjugate. When the cosine in the ring term is expanded, i.e., in the phase with no topological defects, one finds a quasione-dimensional dispersion relation for the excitons (density waves), $\omega_{\mathbf{k}}^{2} \sim \sin ^{2}\left(k_{x} / 2\right) \sin ^{2}\left(k_{y} / 2\right)$. This gapless normal Bose state is expected to be stable against becoming an insulator when $|\mathcal{K}|$ dominates over $\mathcal{U}$.

The ring-only model on the square lattice has special conservation laws: particle numbers on each column and each row are individually conserved. It is this property that is responsible for the vanishing of $\omega_{\mathbf{k}}$ along the lines $k_{x}=0$ and $k_{y}=0$ in the EBL phase. Paramekanti et al. ${ }^{14}$ showed that due to the gapless lines, EBL is a critical (power law) 2D quantum phase with continuously varying exponents.

For our energetics study, it is useful to have a good trial wave function for the EBL phase of hard-core bosons. Motivated by the described "spin wave" theory, we write the following wave function:

$$
\Psi_{\mathrm{EBL}}^{\left(K_{4}<0\right)}\left(\mathbf{r}_{1}, \mathbf{r}_{2}, \ldots\right) \propto \exp \left[-\sum_{i<j} u_{\mathrm{EBL}}\left(\mathbf{r}_{i}-\mathbf{r}_{j}\right)\right],
$$

with

$$
u_{\mathrm{EBL}}\left(\mathbf{r}-\mathbf{r}^{\prime}\right)=\frac{1}{V} \sum_{\mathbf{k}} \frac{\mathcal{W}}{4\left|\sin \left(k_{x} / 2\right)\right|\left|\sin \left(k_{y} / 2\right)\right|} e^{i \mathbf{k} \cdot\left(\mathbf{r}-\mathbf{r}^{\prime}\right)} .
$$

In the spin wave theory, we have $\mathcal{W}=\sqrt{\mathcal{U} /|K|}$, but, more generally, $\mathcal{W}$ is treated as a variational parameter. The summation over $\mathbf{k}$ excludes the lines where either $k_{x}=0$ or $k_{y}$ $=0$. Such wave function can be defined in any sector with fixed number of particles on each row and column as is appropriate in the study of the ring-only model. We find that for moderate $\mathcal{W}$ and away from half filling, this wave function has a density structure factor of the form $\langle\hat{\rho}(-\mathbf{k}) \hat{\rho}(\mathbf{k})\rangle$ $\sim\left|k_{x}\right|\left|k_{y}\right|$ at long wavelengths, as expected in the EBL. Furthermore, the EBL theory predicts that the box correlator defined in Eq. (6) decays as a power law with a continuously varying exponent, and this is what we observe for the wave function. In the energetics study, the parameter $\mathcal{W}$ is varied to minimize the ring energy $H_{4}$.

Returning to the model with $K_{4}>0$, the trial wave function is

$$
\Psi_{\mathrm{EBL}}^{\left(K_{4}>0\right)}\left(\mathbf{r}_{1}, \mathbf{r}_{2}, \ldots\right) \propto(-1)^{N_{\mathrm{I}}} \exp \left[-\sum_{i<j} u_{\mathrm{EBL}}\left(\mathbf{r}_{i}-\mathbf{r}_{j}\right)\right],
$$

where $N_{\mathrm{I}}$ is the number of bosons on the sublattice I of the four sublattices. One can readily translate the preceding results to this wave function, which has the sign structure appropriate for the pure ring exchange model with $K_{4}>0$.

Interestingly, the DLBL wave function in the extreme $d$-eccentricity limit, which was introduced toward the end of Sec. III C and discussed further in Sec. IV B 2, also has the correct sign structure for the ring Hamiltonian. This follows from an observation that this wave function takes opposite signs for any two boson configurations that are connected by $H_{4}$. It is important here that $H_{4}$ contains only unit square plackets, and one subtlety is that such unit ring moves are

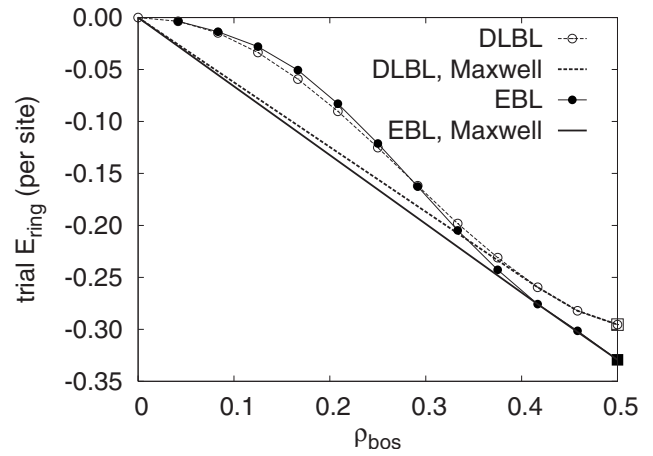

FIG. 14. Trial ring energy in the optimized EBL wave function (filled circles) and extremal DLBL wave function (open circles). Measurements are performed on a $24 \times 24$ system; the boson number is always a multiple of 24 since the extremal DLBL requires that the number of particles are exactly the same in each row and/or column. Away from half filling, the optimized EBL and DLBL wave functions produce uniform liquids, but exactly at half filling, we find a long-range $(\pi, \pi)$ CDW order in both states, which is emphasized with large box symbols. The thin lines are guides for the eyes, while the thick lines are the result of the Maxwell construction for the two trial energies that addresses the possibility of the phase separation at low densities. From this construction, the uniform liquids appear to be stable in the density windows $0.42<\rho<0.50$.

not ergodic in the sectors mentioned earlier with fixed boson numbers on each row and column. The signs of the EBL and extremal DLBL wave functions as written will not agree everywhere. However, in the study of the ring model, it is natural to consider smaller sectors comprising states that are connected by repeated applications of $H_{4}$, and in such sectors, the signs of the two wave functions agree. Thus, one needs to carefully specify what sector restriction is being made when working with these wave functions. As far as the $K_{4}$ energetics is concerned, we find essentially no difference between the wave functions obtained by restricting to the smaller (ergodic) sectors or the larger sectors, which fix only the boson numbers on each row and column. The presented results are all for the latter choice of sectors; the Monte Carlo sampling is performed using arbitrary rectangular ring moves, which are ergodic in this sector.

Figure 14 shows the ring exchange energy per site for the optimized EBL and extremal DLBL wave functions plotted vs boson density. The two trial energies are fairly close; the EBL state has somewhat lower energy for $\rho \geq 1 / 4$, and the difference is largest near $\rho=1 / 2$. Note that the extremal DLBL wave function has no variational parameters, while the EBL state has one parameter. The trial DLBL energy can be somewhat improved by maintaining the same sign structure but taking a variable power of the determinants; such trial energies (which we do not show) approach closer to the optimal EBL energies. Figure 14 suggests that the DLBL wave function not only has the right sign structure, but also has similar short-range correlations to the EBL state. Of course, additional Jastrow-type factors can be used to further improve the DLBL energy, but here and below we emphasize the good sign structure and correlations that are already present in the determinantal construction itself.

The similarity between the EBL and extremal DLBL wave functions is also revealed in their density structure fac- 
tors (see also Sec. IV B 2). In both states away from half filling, for small $\left|k_{x}\right| \ll\left|k_{y}\right|$, we can write $D_{b}\left(k_{x}, k_{y}\right)$ $=A\left(k_{y}\right)\left|k_{x}\right|$. In the DLBL state, $A\left(k_{y}\right)$ appears to be independent of $k_{y}$. On the other hand, in the EBL state, $A\left(k_{y}\right) \sim\left|k_{y}\right|$. Thus, the character of the $k_{x} \rightarrow 0$ singularity is the same in both states for any nonzero $k_{y}$, and it is only in the limit $k_{x}$, $k_{y} \rightarrow 0$ that the two differ. Interestingly, both wave functions also exhibit $2 k_{F}$ singularities in the density structure factor. Such singularities are not apparent in the naive continuum spin wave EBL theory, but manifest themselves in the lattice hard-core boson EBL wave function.

The study of density correlations also reveals that these optimized states at half filling, in fact, have spontaneous $(\pi, \pi)$ charge order, while away from half filling, no order is observed. Thus, even though in the present crude energetics work we do not directly consider conventional ordered states of bosons such as charge or bond density waves, the EBL and DLBL wave functions provide some access to the energetics of such ordered states. (Note, however, that these trial states likely have some degree of EBL-ness, e.g., finite compressibility; this is analogous to the well-known observation that when a conventional Jastrow wave function is driven into a regime of charge order, it in fact represents a supersolid that also supports off-diagonal long-range order.) As we have already mentioned, Monte Carlo studies ${ }^{16,17}$ suggest staggered CDW at $\rho=1 / 2$, and our finding of the same tendency in the considered wave functions lends some support to the goodness of the variational approach. Conventional boson orders are potentially important near other commensurate boson densities, but are less important at generic $\rho$, and we will not consider charge ordered states further.

Leaving the charge orders aside, an important feature is prominent in the energy plots in Fig. 14 for the considered uniform liquids. We see regions where $\epsilon(\rho)$ is concave, which signals an instability toward phase separation. Using the Maxwell construction for the available data, we conclude that the system phase separates for boson densities $\rho \leqq 0.42$. At low densities, the system will spontaneously separate into an empty phase and the EBL phase at density $\rho=0.42$. In the region near $\rho=1 / 2$, the considered uniform liquids appear to be stable, except perhaps very close to half filling.

The tendency in the ring model toward phase separation at low densities was noted by Rousseau et al. ${ }^{17}$ Indeed, if we consider two bosons on an otherwise empty lattice, the lowest ring energy is achieved when the two reside on the same square placket, since only in this case the ring term is nonzero. Thus, two bosons can be bound together by the ring energy. By performing exact diagonalization of small clusters up to $4 \times 4$ with open boundary conditions and for different boson numbers, we find that the energy per particle is the lowest when the density is near half filling; also, larger open clusters achieve lower energies per boson. One can construct eigenstates of the ring Hamiltonian at low densities composed of such isolated clusters, and the discussed tendencies suggest that not only two but many bosons tend to clump together in the presence of the ring exchanges.

One more notable feature in the energy plots occurs near half filling. We show only the $\rho \leqslant 1 / 2$ part, while the $\rho$ $\geqslant 1 / 2$ part would be the mirror image due to particle-hole symmetry. Therefore, $\epsilon(\rho)$ shows a cusp precisely at half filling - at least in the EBL case and for the system sizes studied. Since $\mu=d \epsilon / d \rho$, the cusp may be interpreted as an evidence for a charge gap at this density, which appears to be in line with the proposal of the CDW order by the Monte Carlo studies.

We do not know whether the above observations persist in the limit of large systems. Thus, it may also happen that there will be no regime of the EBL phase in the specific model if the system chooses to phase separate into empty space and a half filled charge insulator. If there is a stable EBL regime over some density window near half filling, then the phase separation at lower densities is likely to be into empty space and an EBL phase of an appropriate density determined from the Maxwell construction. The existence of an EBL phase near half filling could presumably be checked by careful Monte Carlo simulations for the pure ring model. It is also plausible that the regime of the stable uniform EBL phase could be extended by adding interactions that disfavor phase separation, but we have not explored this in the present work.

\section{B. Full $J-K_{4}$ Hamiltonian}

We now turn to the case with nonzero boson hopping $J$ $>0$. Consider first small $J$, and suppose we start in the uniform $\mathrm{EBL}$ or DLBL regime assuming such exists. As we have discussed, the hopping and ring terms frustrate one another. At present, we do not know how to include the effects of hopping if we begin with the EBL. Thus, the EBL wave function is defined in sectors with fixed boson numbers on each row and/or column, but what relative signs should we take for the different sectors that are now mixed by the boson hopping? If, for example, we naively extend Eq. (116) to all boson configurations, with perhaps modified Jastrow factors, the expectation value of $H_{J}$ remains precisely zero. It seems that one needs to change the sign structure of the wave function in order to treat the frustration accurately.

On the other hand, the DLBL wave function provides a natural continuation from the extremal case of the ring-only model, and we find that it is indeed capable of utilizing both the ring energy and the hopping energy. More precisely, for nonzero $J$, the optimized $d$ eccentricity is finite and varies as $J$ is increased, moving toward zero $d$ eccentricity at large $J$. Within our restricted variational energetics study, this is the strongest evidence we can offer that the ground state of the ring Hamiltonian in this regime is in the DLBL phase.

Some details of the variational study are summarized in the "phase diagram" in Fig. 15. At a given density, the trial energy of the DBL state is optimized using the ratio $t_{\perp} / t_{\|}$as a variational parameter. Similar to the pure ring model, we also analyze the possibility of phase separation, which is done in the spirit of Fig. 14. We find a stable uniform DBL phase in the density range roughly similar to the ring-only model. Physically, we expect the phase separation to be suppressed when the hopping becomes nonzero, but our study is too limited to explore this systematically.

The phase diagram in Fig. 15 also includes a conventional superfluid state of bosons, which is expected in the regime of 


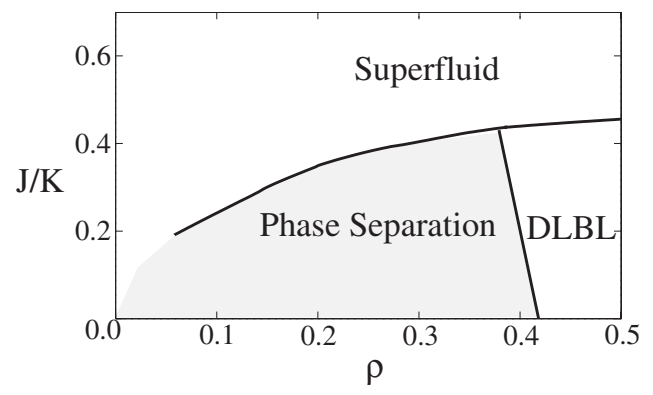

FIG. 15. Schematic "variational phase diagram" of the $J-K_{4}$ model. Numerics is done for the same sizes as in Fig. 14. The phase boundaries are obtained by examining the DBL state, allowing for the possibility of phase separation, and the superfluid state (the latter appears to be always uniform in our study). The optimal values of $t_{\perp} / t_{\|}$throughout the whole DBL phase are such that the fermions have open Fermi surfaces; i.e., this is the DLBL phase of Sec. III C.

large $J$. We take the Jastrow-type form for the trial wave function [Eq. (16)] and use the pseudopotential $u\left(\mathbf{r}-\mathbf{r}^{\prime}\right)$ $=W /\left|\mathbf{r}-\mathbf{r}^{\prime}\right|^{p}$, with two variational parameters $W$ and $p$. Allowing more parameters such as an independent nearestneighbor pseudopotential does not visibly change the optimized energies. Interestingly, as deduced from the energetics, the superfluid-DBL phase boundary roughly coincides with the limit of vanishing $d$ eccentricity $t_{\perp}=t_{\|}$. The resulting $(\mathrm{det})^{2}$ wave function is positive, and, as we discussed at length in Sec. IV, this state has off-diagonal longrange order. Of course, it does not have the same longwavelength correlations as the superfluid (e.g., it has unusual $2 k_{F}$ density correlations), but it appears to have reasonable short-range correlations.

Finally, we also remark that in the present $J-K_{4}$ study, the optimal DBL phase when it wins over the superfluid is, in fact, the DLBL phase with open Fermi surfaces discussed in Sec. III C and is in the regime where the boson Green's function decays exponentially in all directions. As we discussed in Secs. III and IV, despite such "locality" in the boson correlator, this unusual phase is still critical with many low-energy excitations.

Summarizing, the DBL wave function is able to interpolate between the EBL and the superfluid regimes in the frustrated $J-K_{4}$ model. This is the main argument supporting our proposal that the ground state of the ring Hamiltonian in the intermediate regime is in the DLBL phase.

\section{SUMMARY AND DISCUSSION}

Our main results were summarized in Sec. I, so here we only highlight the most interesting points and discuss possible future directions. We are searching for examples of uncondensed quantum boson liquids that respect time reversal and occur at generic continuously varying densities. In this paper, we proposed several wave functions that produce such liquids and studied in detail the specific states dubbed DBL and DLBL. While the initial wave function construction appears somewhat ad hoc, we argued that it is no more so than the slave-particle construction of spin liquid states in frus- trated antiferromagnets, which has been developed to maturity over the past two decades. We introduced a particular slave-fermion treatment of hard-core bosons on a square lattice that corresponds to the proposed DBL wave functions. This approach allows to go beyond the trial ground state wave function, suggesting natural excitations, and eventually leads to a gauge theory description of the low-energy properties of the putative boson liquid phase. Using techniques previously developed for the so-called uniform RVB spin liquid with a Fermi sea of spinons, we argued for the possible stability of the DBL phases to gauge fluctuations and found the DLBL regime to be particularly stable. Within the gauge theory formulation, we also analyzed boson and boson density correlators at long distances, which reveal special singular surfaces in the momentum space.

The singular surfaces were confirmed by directly measuring the properties of the proposed DBL/DLBL wave functions; in the same numerics, no potential orders were observed, indicating stability of the states. We remark here that the Gutzwiller wave functions and the gauge theory should not be viewed as identical descriptions, and, in particular, the detailed long-distance properties may be different. The Gutzwiller wave functions can be used for crude energetics and qualitative characterization, but in our opinion the gauge theory is more complete as far as the actual quantum phase is concerned. Indeed, the wave functions as constructed do not incorporate any dynamics of the gauge fluxes, which are clearly additional low-energy "variational" degrees of freedom that the quantum system is going to explore and utilize.

From the singular momentum surfaces in the boson properties, one can, in principle, recover the underlying Fermi surfaces of the slave-particle construction; the latter have correct volumes for the boson density, offering a tantalizing possibility of some "Luttinger theorem" for uncondensed liquids of interacting bosons.

Some striking thermodynamic properties of the proposed boson liquids follow from their fermion-gauge character. ${ }^{5,31-40}$ In both the DBL and DLBL phases, because of the gapless Fermi surfaces and the gauge interactions, the specific heat at low temperatures is expected to behave as $C \sim T^{2 / 3}$, while the resistivity is expected to vanish as $R_{b}$ $\sim T^{4 / 3}$. The last result in the DBL phase with closed Fermi surfaces is simply a quote from earlier studies of such Fermi sea-gauge systems, ${ }^{34,35}$ while the case with open Fermi surfaces requires slightly more care. Our argument is based on the application of the Ioffe-Larkin rule, ${ }^{4}$ which gives the boson resistivity as $R_{b}=R_{d_{1}}+R_{d_{2}}$. For the Fermi surfaces as in the right panel of Fig. 1, and the conductivity measured, say, in the $\hat{\mathbf{x}}$ direction, the small-angle scattering by the gauge field can effectively degrade the $d_{2}$ fermion current; therefore the standard result is $R_{d_{2}} \sim T^{4 / 3}$. On the other hand, such scattering cannot completely degrade the $d_{1}$ fermion current, so $R_{d_{1}} \ll R_{d_{2}}$, and $R_{b} \sim T^{4 / 3}$ follows. (Parenthetically, the $d_{1}$ current can be degraded by quartic or more particle interactions, but such contributions vanish as $T^{2}$ or faster.)

Intriguingly, in the DLBL phase, the boson correlators are expected to be short ranged despite such manifest thermodynamic signatures of gaplessness and criticality. On the other hand, the boson box correlator in the DLBL decays as a 


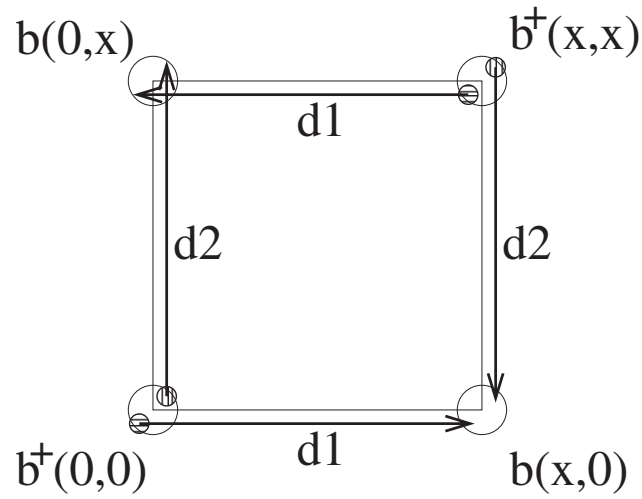

FIG. 16. Boson box correlator $\left\langle b^{\dagger}(0,0) b^{\dagger}(x, x) b(x, 0) b(0, x)\right\rangle$ in the DLBL phase reveals the fermionic character of the partons, decaying as power law and having negative sign from the fermion exchange.

power law and is negative $\left(-x^{-8}\right)$, offering perhaps a more physical glimpse of the fermionic partons in this phase. Indeed, consider inserting bosons at $(0,0)$ and $(x, x)$ and removing at $(x, 0)$ and $(0, x)$. The dominant contribution to the box correlator comes from the $d_{1}$ fermions propagating $(0,0) \rightarrow(x, 0)$ and $(x, x) \rightarrow(0, x)$, while the $d_{2}$ paths are $(0,0) \rightarrow(0, x)$ and $(x, x) \rightarrow(x, 0)$, which is illustrated in Fig. 16. The minus sign of the box correlator is then due to one fermionic exchange needed so that the two pairs of injected fermions are removed with the same pairing.

The proposed DBL/DLBL wave functions do not satisfy Marshall signs, and their interesting sign structure is brought out by the nodal pictures in the continuum such as Fig. 2, which give some caricature of the wave function signs also on the lattice even though the nodes are not sharply defined in this case. Clearly, these wave functions cannot be ground states of boson models without frustration. To address the question of what Hamiltonians may stabilize such phases, we considered a particular frustrated hard-core boson model with competing hopping and four-site ring exchange terms, and our energetics study suggests that the DLBL state is a good candidate in this model near half filling. Unfortunately, the frustrated nature of the boson motion makes this model not suitable for large system quantum Monte Carlo studies. We still suggest it as an interesting model for numerical studies such as exact diagonalization of small systems or DMRG; the gapless nature of the boson liquid may make results hard to interpret, but the short-range character of the correlations and particularly the quasilocal nature of the DLBL phase can perhaps facilitate DMRG to access larger systems.

Looking ahead, we now describe one of the main drives behind the study of uncondensed boson liquids, despite its own intrinsic interest. We are searching for electronic conducting non-Fermi-liquids, and the ideas of the present work suggest some avenues in this direction. As a specific example, consider the slave boson approach that is popular in the context of the $t-J$ model of high- $T_{c}$ superconductors. The electron operator is written as $c_{\sigma}^{\dagger}=b^{\dagger} f_{\sigma}^{\dagger}$, leading to a theory of spinons and slave bosons strongly coupled via an emergent fluctuating gauge field. Phenomenologically, a Fermi sea of spinons is very appealing in the strange metal, but what about the slave bosons? If they condense, we would recover the conventional Fermi liquid. It has been argued that the fluctuating gauge field frustrates the motion of the bosons and suppresses the tendency to condense. ${ }^{4-6,34,35}$ If this suppression could persist to zero temperature, we would obtain a conducting non-Fermi-liquid state. Such a possibility is precisely what we are trying to establish in the present work, and our approach would be to write the slave boson in terms of other (second generation) slave fermions, $b^{\dagger}=d_{1}^{\dagger} d_{2}^{\dagger}$.

On the level of wave functions, we would then write an electronic state of the form

$$
\Psi_{\text {electron }}(\uparrow, \downarrow)=\left[(\operatorname{det})_{x} \times(\operatorname{det})_{y}\right](\uparrow, \downarrow) \operatorname{det}(\uparrow) \operatorname{det}(\downarrow),
$$

where schematically $(\uparrow)$ or $(\downarrow)$ denotes locations of all electrons with spin up or spin down, respectively. Each determinant in the slave boson wave function $(\text { det })_{x} \times(\text { det })_{y}$ evaluates appropriate $d$-particle orbitals at the locations of all electrons irrespective of their spin, which assures the nodouble-occupancy constraint. There is significant freedom in specifying these orbitals, and whether such wave functions are useful for any electronic system requires detailed energetics studies of specific Hamiltonians. One can, nevertheless, develop a low-energy theory of such a $T=0$ phase in the spirit of the present paper, thereby obtaining an itinerant nonFermi-liquid conducting phase. If the DBL or DLBL bosonic states studied in this work appear as useful caricatures of the behavior of the electronic charge, we may even call the resulting phase a " $d$-wave metal"! Such all-fermion description of electrons on the square lattice with no double occupancy has some phenomenological appeal, but detailed explorations are left for future work.

\section{ACKNOWLEDGMENTS}

We would like to acknowledge discussions with T. Senthil and A. Vishwanath, and thank Jason Alicea for help with some calculations. The work at KITP was supported by the National Science Foundation through Grants No. PHY9907949 and No. DMR-0529399.
${ }^{1}$ P. W. Anderson, Science 235, 1196 (1987).

${ }^{2}$ G. Baskaran, Z. Zou, and P. W. Anderson, Solid State Commun. 63, 973 (1987).

${ }^{3}$ G. Kotliar and J. Liu, Phys. Rev. B 38, 5142 (1988).

${ }^{4}$ L. B. Ioffe and A. I. Larkin, Phys. Rev. B 39, 8988 (1989).
${ }^{5}$ P. A. Lee, N. Nagaosa, and X.-G. Wen, Rev. Mod. Phys. 78, 17 (2006).

${ }^{6}$ M. V. Feigelman, V. B. Geshkenbein, L. B. Ioffe, and A. I. Larkin, Phys. Rev. B 48, 16641 (1993).

${ }^{7}$ D. Dalidovich and P. Phillips, Phys. Rev. B 64, 052507 (2001). 
${ }^{8}$ V. M. Galitski, G. Refael, M. P. A. Fisher, and T. Senthil, Phys. Rev. Lett. 95, 077002 (2005).

${ }^{9}$ J. Alicea, O. I. Motrunich, and M. P. A. Fisher, Phys. Rev. Lett. 95, 247203 (2005); Phys. Rev. B 73, 174430 (2006).

${ }^{10}$ D. M. Ceperley, J. Stat. Phys. 63, 1237 (1991).

${ }^{11}$ X.-G. Wen, Phys. Rev. B 65, 165113 (2002).

${ }^{12}$ S. M. Girvin and A. H. MacDonald, Phys. Rev. Lett. 58, 1252 (1987).

${ }^{13}$ C. L. Kane, S. Kivelson, D. H. Lee, and S. C. Zhang, Phys. Rev. B 43, 3255 (1991).

${ }^{14}$ A. Paramekanti, L. Balents, and M. P. A. Fisher, Phys. Rev. B 66, 054526 (2002).

${ }^{15}$ A. W. Sandvik, S. Daul, R. R. P. Singh, and D. J. Scalapino, Phys. Rev. Lett. 89, 247201 (2002).

${ }^{16}$ R. G. Melko, A. W. Sandvik, and D. J. Scalapino, Phys. Rev. B 69, 100408(R) (2004).

${ }^{17}$ V. G. Rousseau, R. T. Scalettar, and G. G. Batrouni, Phys. Rev. B 72, 054524 (2005); V. Rousseau, G. G. Batrouni, and R. T. Scalettar, Phys. Rev. Lett. 93, 110404 (2004).

${ }^{18}$ S. C. Zhang, T. H. Hansson, and S. Kivelson, Phys. Rev. Lett. 62, 82 (1989).

${ }^{19}$ D. H. Lee and M. P. A. Fisher, Phys. Rev. Lett. 63, 903 (1989).

${ }^{20}$ X. G. Wen, Phys. Rev. B 43, 11025 (1991); Phys. Rev. Lett. 64, 2206 (1990).

${ }^{21}$ X.-G. Wen, Phys. Rev. B 60, 8827 (1999).

${ }^{22}$ V. Kalmeyer and R. B. Laughlin, Phys. Rev. B 39, 11879 (1989); Phys. Rev. Lett. 59, 2095 (1987).

${ }^{23}$ X. G. Wen, F. Wilczek, and A. Zee, Phys. Rev. B 39, 11413 (1989).

${ }^{24}$ R. B. Laughlin and Z. Zou, Phys. Rev. B 41, 664 (1990).

${ }^{25}$ Y. Zhou and X.-G. Wen, arXiv:cond-mat/0210662 (unpublished).

${ }^{26}$ S. Yunoki and S. Sorella, Phys. Rev. B 74, 014408 (2006).

${ }^{27}$ W. Rantner and X.-G. Wen, Phys. Rev. B 66, 144501 (2002).
${ }^{28}$ M. Hermele, T. Senthil, M. P. A. Fisher, P. A. Lee, N. Nagaosa, and X.-G. Wen, Phys. Rev. B 70, 214437 (2004); M. Hermele, T. Senthil, and M. P. A. Fisher, ibid. 72, 104404 (2005).

${ }^{29}$ D. M. Ceperley, G. V. Chester, and M. H. Kalos, Phys. Rev. B 16, 3081 (1977).

${ }^{30}$ F. C. Zhang, C. Gros, T. M. Rice, and H. Shiba, Supercond. Sci. Technol. 1, 36 (1988); arXiv:cond-mat/0311604.

${ }^{31}$ T. Holstein, R. E. Norton, and P. Pincus, Phys. Rev. B 8, 2649 (1973).

${ }^{32}$ M. Y. Reizer, Phys. Rev. B 40, 11571 (1989).

${ }^{33}$ P. A. Lee, Phys. Rev. Lett. 63, 680 (1989).

${ }^{34}$ L. B. Ioffe and G. Kotliar, Phys. Rev. B 42, 10348 (1990).

${ }^{35}$ P. A. Lee and N. Nagaosa, Phys. Rev. B 46, 5621 (1992).

${ }^{36}$ J. Polchinski, Nucl. Phys. B 422, 617 (1994).

${ }^{37}$ B. L. Altshuler, L. B. Ioffe, and A. J. Millis, Phys. Rev. B 50, 14048 (1994).

${ }^{38}$ C. Nayak and F. Wilczek, Nucl. Phys. B 417, 359 (1994); 430, 534 (1994)

${ }^{39}$ Y. B. Kim, A. Furusaki, X. G. Wen, and P. A. Lee, Phys. Rev. B 50, 17917 (1994); Y. B. Kim, P. A. Lee, and X. G. Wen, ibid. 52, 17275 (1995).

${ }^{40}$ T. Senthil, M. Vojta, and S. Sachdev, Phys. Rev. B 69, 035111 (2004).

${ }^{41}$ V. M. Galitski, Phys. Rev. B 72, 214201 (2005).

${ }^{42}$ The structure of the $1 / N$ diagrams for the particle-hole and particle-particle vertices, Figs. 11 and 12, is specific to our $\mathrm{N}$-flavor extension defined in Sec. V C, while different extensions can lead to different organizations of diagrams.

${ }^{43}$ R. Shankar, Rev. Mod. Phys. 66, 129 (1994).

${ }^{44}$ J. Polchinski, in Recent Directions in Particle Theory, Proceedings of TASI 1992, edited by J. Harvey and J. Polchinski (World Scientific, Singapore, 1993); arXiv:hep-th/9210046. 